\title{
Five new and five known species of the genus Tylencholaimus de Man, 1876 (Nematoda: Dorylaimida: Tylencholaimoidea) from Western Ghats of India
}

\author{
Md Niraul ISLAM ${ }^{\circledR}{ }^{1, *} \&$ Wasim AHMAD $^{2}$ \\ ${ }^{1,2}$ Nematode Biodiversity Research Lab, Department of Zoology, \\ Aligarh Muslim University, Aligarh-202002, India. \\ ${ }^{*}$ Corresponding author: mnislam15@gmail.com \\ 22ahmadwasim57@gmail.com \\ ${ }^{1}$ urn:lsid:zoobank.org:author:A202F47B-7E49-43AE-A2EF-988E7E891AA9 \\ ${ }^{2}$ urn:1sid:zoobank.org:author:830C4201-DE41-4B24-8280-4C4DCC0C89EF
}

\begin{abstract}
Five new and five known species of the genus Tylencholaimus de Man, 1876 are described from the Western Ghats of India. Tylencholaimus macroamphidius sp. nov. has a $0.61-0.85 \mathrm{~mm}$ long body, angular lips, large amphid, 8.0-9.0 $\mu \mathrm{m}$ long odontostyle, odontophore with asymmetrical basal knobs, pharyngeal bulb expanding gradually, female genital system mono-prodelphic, and a rounded to conoid tail. Tylencholaimus shamimi sp. nov. has a $0.57-0.71 \mathrm{~mm}$ long body, $6.0-7.0 \mu \mathrm{m}$ long odontostyle, odontophore with basal thickening, pharyngeal bulb expanding gradually, female genital system amphidelphic and a convex-conoid tail. Tylencholaimus southindicus sp. nov. has a $0.44-0.55$ $\mathrm{mm}$ long body, lip region with distinct labial disc, 4.5-5.5 $\mu \mathrm{m}$ long odontostyle, odontophore with minute basal knobs, pharyngeal expansion abrupt, female genital system mono-prodelphic, and a roundedconoid to rounded tail. Tylencholaimus striatus sp. nov. has a $0.30-0.34 \mathrm{~mm}$ long body with distinctly striated cuticle, 5.0-5.5 $\mu \mathrm{m}$ long odontostyle, odontophore with minute basal knobs, pharyngeal bulb expanding abruptly, female genital system mono-prodelphic, and a conoid tail with bluntly rounded terminus. Tylencholaimus tamiliensis sp. nov. has $0.51-0.58 \mathrm{~mm}$ long body, 5.5-6.0 $\mu \mathrm{m}$ long odontostyle, odontophore with minute basal knobs, pharyngeal bulb expanding gradually, female genital system mono-prodelphic and tail rounded to conoid with sunken terminus. Five known species of the genus Tylencholaimus viz. T. mirabilis, T. teres, T. micronanus, T. ibericus and T. cosmos also recorded from the region and redescribed/illustrated.
\end{abstract}

Keywords. Nematode, description, tylencholaimid, biodiversity hotspot.

Islam M.N. \& Ahmad W. 2021. Five new and five known species of the genus Tylencholaimus de Man, 1876 (Nematoda: Dorylaimida: Tylencholaimoidea) from Western Ghats of India. European Journal of Taxonomy 774: 58-105. https://doi.org/10.5852/ejt.2021.774.1531

\section{Introduction}

The Western Ghats in India is recognized as a biodiversity hotspot (Myers et al. 2000) and is one of the world's eight "Hottest biodiversity hotspots". It represents some of the best tropical evergreen forests 
with highly mosaic topography. Its climate varies with altitudinal gradation and equatorial distance. Although, the Western Ghats covers about $6 \%$ of the total Indian land area, it retains very rich floral and faunal diversity (CEPF 2007). Several species of soil-inhabiting nematodes have been described from this biodiversity hotspot (Ferris et al. 1979; Ahmad \& Jairajpuri 1982, 1983, 1984, 1986, 1988; Ahmad et al. 1992; Ahmad \& Ahmad 1992, 2002; Ahmad 1993; Dhanam \& Jairajpuri 1999; Tabinda et al. 2013), however, the species diversity of the genus Tylencholaimus de Man, 1876 which is one of the most speciose genus representing the superfamily Tylencholaimoidea Filipjev, 1934 of the order Dorylaimida Pearse, 1942, has not been extensively studied in this region. The nematodes belonging to this genus mostly occur in undisturbed forest soils and have worldwide distribution. Several authors (Jairajpuri 1965; Loof \& Jairajpuri 1968; Ali \& Chisty 1972; Ahmad \& Jairajpuri 1979; Khan \& Laha 1982; Rahman et al. 1987; Khan et al. 1989; Dhanachand 1994; Khan \& Ahmad 1994; Dhanam \& Jairajpuri 1999; Mohilal \& Dhanachand 2003; Mushtaq et al. 2007 and Ahad \& Ahmad 2016) added species to this genus from India, but currently there is not much information from the Western Ghats. Only three species viz., Tylencholaimus cosmos Peña-Santiago, 2008, T. ibericus Peña-Santiago \& Coomans, 1994 and T. vulvulatus Rahman et al., 1987 have been recorded so far (Dhanam \& Jairajpuri 1999) from this region.

During the present study, several populations representing the genus Tylencholaimus were collected from different localities of this region. After examination, they were found to represent five new and five known species, which are described and illustrated in this paper.

\section{Material and methods}

Soil samples were collected from different localities of the Western Ghats of India. The nematodes were extracted from soil samples following Cobb's (1918) sieving and decantation and modified Baermann's funnel techniques. The extracted nematodes were fixed in hot triethanolamine-glycerol fixative, dehydrated by the slow evaporation method (Seinhorst 1959), and mounted in anhydrous glycerine. Permanent mounts were prepared using the paraffin wax ring method (de Maeseneer \& d'Herde 1963). The measurements were taken using an ocular micrometre, and position of pharyngeal gland nuclei and their orifices were calculated according to Loof \& Coomans (1970). Line drawings were prepared using a drawing tube, and photographs were taken with a Nikon DS digital Camera attached with Nikon Eclipse $80 \mathrm{i}$ microscope. Raw photographs were edited using Adobe ${ }^{\circledR}$ Photoshop ${ }^{\circledR}$.

Type and others specimens are deposited in the nematode collection of the Department of Zoology, Aligarh Muslim University, India (AMU/ZD/NC), as well as in the nematode collection of the Zoological Survey of India, Kolkata, India.

\section{List of abbreviations}

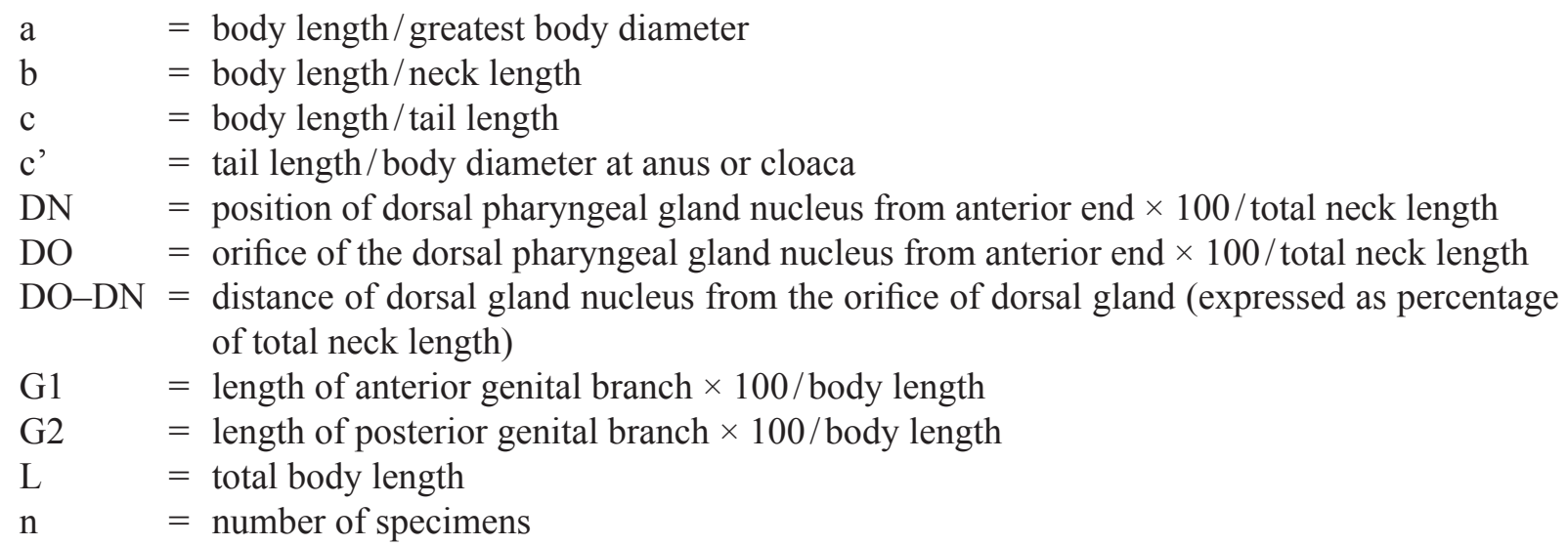




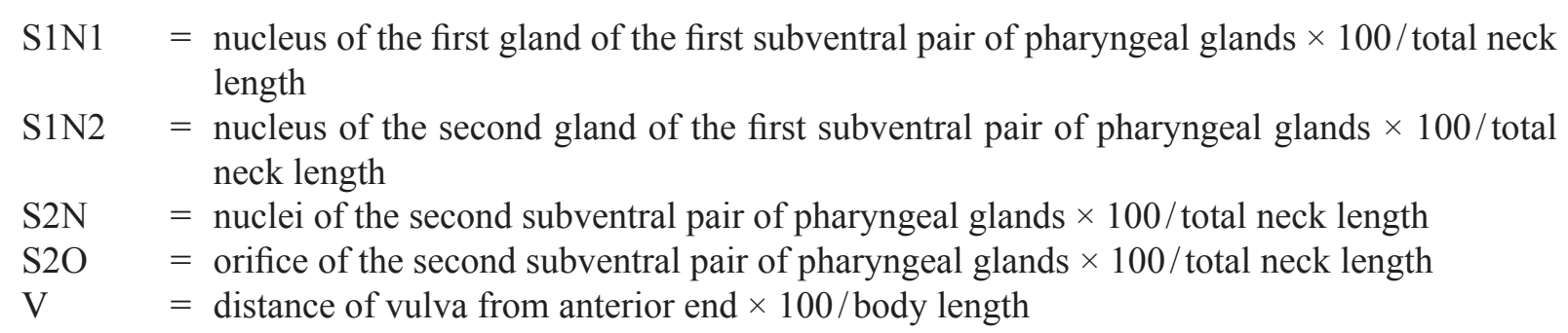

\section{Results}

Phylum Nematoda Cobb, 1932

Class Enoplea Inglis, 1983

Subclass Dorylaimia Inglis, 1983

Order Dorylaimida Pearse, 1942

Superfamily Tylencholaimoidea Filipjev, 1934

Family Tylencholaimidae Filipjev, 1934

Subfamily Tylencholaiminae Filipjev, 1934

Genus Tylencholaimus de Man, 1876

Tylencholaimus mirabilis (Bütschli, 1873)

Figs 1-2, Table 1

Tylenchus mirabilis Bütschli, 1873: 44-45.

Discomyctus brevicaudatus Tarjan, 1953: 52-54.

Tylencholaimus mirabilis - de Man 1876: 43; 1880: 66; 1884: 104-105. — Loof \& Jairajpuri 1968: 321-325. — Vinciguerra 1986: 112. — Peña-Santiago \& Coomans 1994b: 199-206.

Dorylaimellus mirabilis - Thorne 1939: 140.

Dorylaimellus (Tylencholaimus) mirabilis - Meyl 1953: 94

Tylencholaimus brevicaudatus - Tarjan 1956: 91. — Loof 1961: 246-247. — Coomans 1962: 146-149.

\section{Material examined}

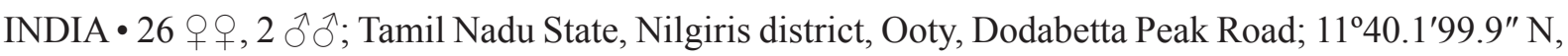
76073.5'36.9" E; 10-15 cm depth; 28 Mar. 2018; soil samples collected from around the roots of shrubs (unidentified); slides reference number AMU/ZD/NC/Tylencholaimus mirabilis/1-10.

\section{Description}

Female

Small sized nematodes, slightly curved ventrad upon fixation; body cylindrical, tapering gradually towards both extremities but more so towards the anterior end. Cuticle with two distinct layers, 1.0 $1.5 \mu \mathrm{m}$ thick at anterior region, $2.0-2.5 \mu \mathrm{m}$ at midbody and $3.0-4.0 \mu \mathrm{m}$ on tail. Outer cuticle thin, finely striated; inner layer thick, its outline somewhat irregular, with distinct radial refractive elements. Lateral chords occupying about $15-25 \%$ of midbody diameter. Body pores distinct, lateral pores: one at odontostyle-odontophore region, $2-5$ in neck region, 5-8 at pharyngeal base to vulva and 5-10 in post-vulval region; dorsal body pores: one at odontostyle-odontophore region, 1-3 in neck region, 3 from pharyngeal base to vulva and 1-2 in post-vulval region; ventral body pores: $1-3$ in neck region, 1-3 from pharyngeal base to vulva and 3 in post-vulval region. Lip region cap-like, offset by deep constriction, 1.8-2.2 times as wide as high or about $1 / 3$ of the body diameter at neck base. Lips rounded, amalgamated, inner part slightly elevated. Labial and cephalic papillae distinct but not interfering with the labial contour. Amphids cup-shaped, their aperture occupying about $1 / 3$ to $1 / 2$ of lip region diameter. 


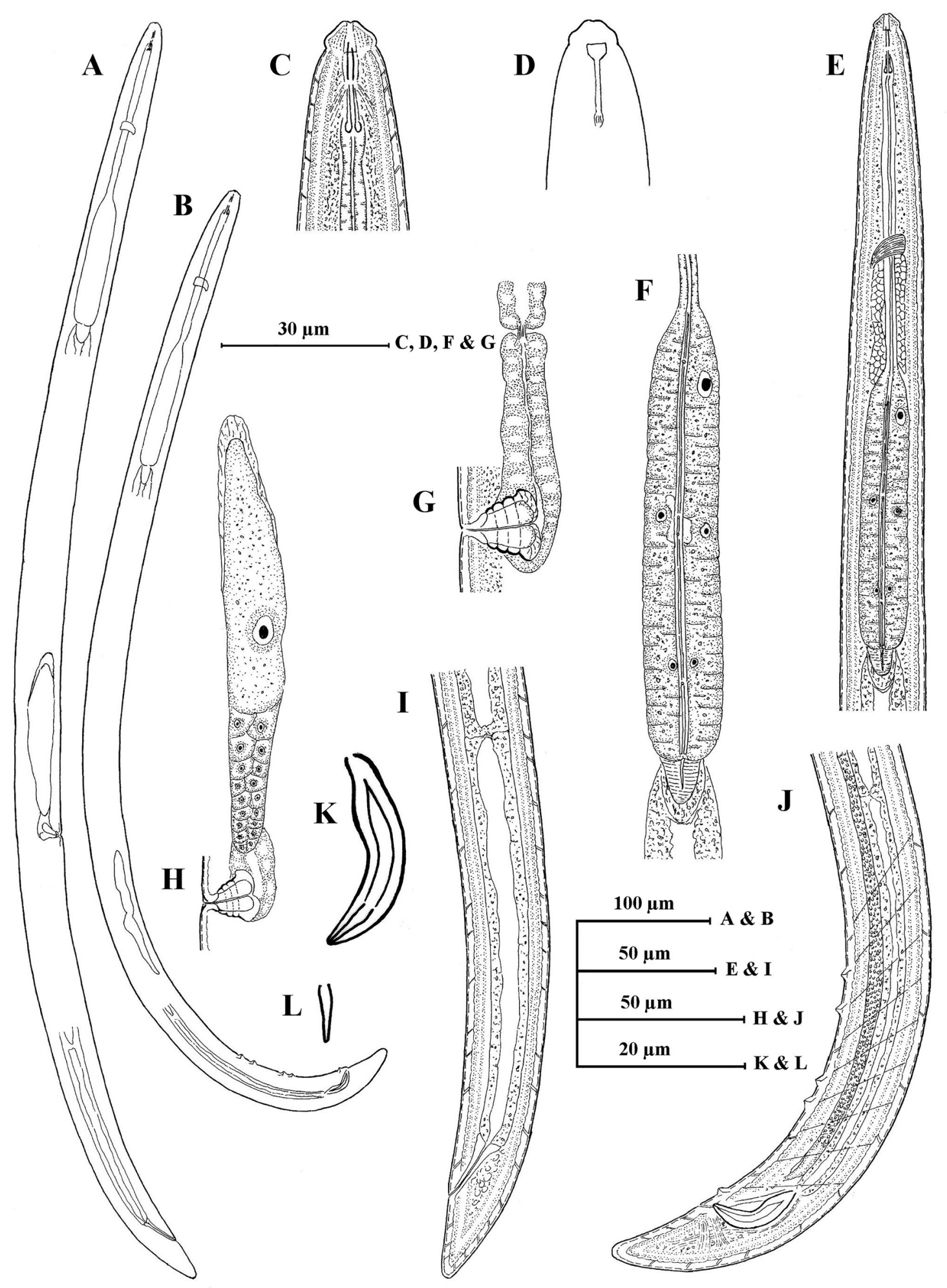

Fig. 1. Tylencholaimus mirabilis (Bütschli, 1873). A. Entire female. B. Entire male. C. Female anterior region. D. Female anterior region showing amphid. E. Female pharyngeal region. F. Female expanded part of pharynx. G-H. Female genital system. I. Female posterior region. J. Male posterior region. K. Spicule. L. Lateral guiding piece. 
Table 1. Measurements of Tylencholaimus mirabilis (Bütschli, 1873). All measurements are in $\mu \mathrm{m}$ and in the form: mean \pm s.d. (range) for females, and measurements for each male $(n=2)$ separated by a comma.

\begin{tabular}{|c|c|c|}
\hline Characters & Females & Males \\
\hline $\mathrm{n}$ & 26 & 2 \\
\hline $\mathrm{L}$ & $872 \pm 50.6(777-978)$ & 914,803 \\
\hline a & $27.2 \pm 1.4(25-30)$ & $29.3,29.2$ \\
\hline $\mathrm{b}$ & $3.8 \pm 0.19(3.4-4.6)$ & $4.1,4.2$ \\
\hline $\mathrm{c}$ & $28.2 \pm 2.0(25-33)$ & $30.2,28.1$ \\
\hline$c^{\prime}$ & $1.4 \pm 0.10(1.2-1.8)$ & $1.4,1.4$ \\
\hline V & $60.8 \pm 1.4(59.2-64.4)$ & - \\
\hline G1 & $14.0 \pm 1.1(12.4-16.2)$ & - \\
\hline G2 & $0.83 \pm 0.13(0.62-1.1)$ & - \\
\hline Body diameter at neck base & $29.5 \pm 1.5(26-32)$ & 32,29 \\
\hline Body diameter at mid body & $30.3 \pm 1.7(27-33)$ & 32,30 \\
\hline Body diameter at anus & $18.6 \pm 1.1(17-21)$ & 20,21 \\
\hline Lip region diameter & $8.8 \pm 0.37(8.0-10)$ & $9.0,9.0$ \\
\hline Lip region height & $4.4 \pm 0.27(4.0-5.0)$ & $5.0,4.0$ \\
\hline Amphidial aperture & $3.4 \pm 0.27(3.0-4.0)$ & $4.0,4.0$ \\
\hline Odontostyle length & $6.8 \pm 0.49(6.5-8.0)$ & $8.0,7.5$ \\
\hline Odontophore length & $10.6 \pm 0.49(8.5-11.0)$ & $10,9.0$ \\
\hline Total stylet length & $17.2 \pm 0.44(16.5-19.0)$ & $18,16.5$ \\
\hline Guiding ring from anterior end & $6.2 \pm 0.72(5.0-7.0)$ & $5.0,7.0$ \\
\hline Nerve ring from anterior end & $78.5 \pm 3.5(76-86)$ & 81,74 \\
\hline Neck length & $210.1 \pm 10.2(180-238)$ & 220,217 \\
\hline Expanded part of pharynx & $94.5 \pm 4.6(84-105)$ & 80,93 \\
\hline Cardia length & $10.1 \pm 0.90(8.0-12.0)$ & $10.5,11.5$ \\
\hline Anterior genital branch & $124.7 \pm 10.9(107-144)$ & - \\
\hline Posterior genital branch & $7.3 \pm 1.06(5.0-8.5)$ & - \\
\hline Vaginal length & $13.1 \pm 0.72(11-15)$ & - \\
\hline Vulva from anterior end & $541.4 \pm 31.6(494-612)$ & - \\
\hline Prerectum length & $147.9 \pm 16.8(120-186)$ & 127,139 \\
\hline Rectum length & $26.9 \pm 2.7(21-31)$ & 26,30 \\
\hline Tail length & $29.2 \pm 1.8(26-32)$ & 29,30 \\
\hline Spicules length & - & 27,27 \\
\hline Lateral guiding pieces & - & $6.0,6.5$ \\
\hline Ventromedian supplements & - & 4,4 \\
\hline
\end{tabular}



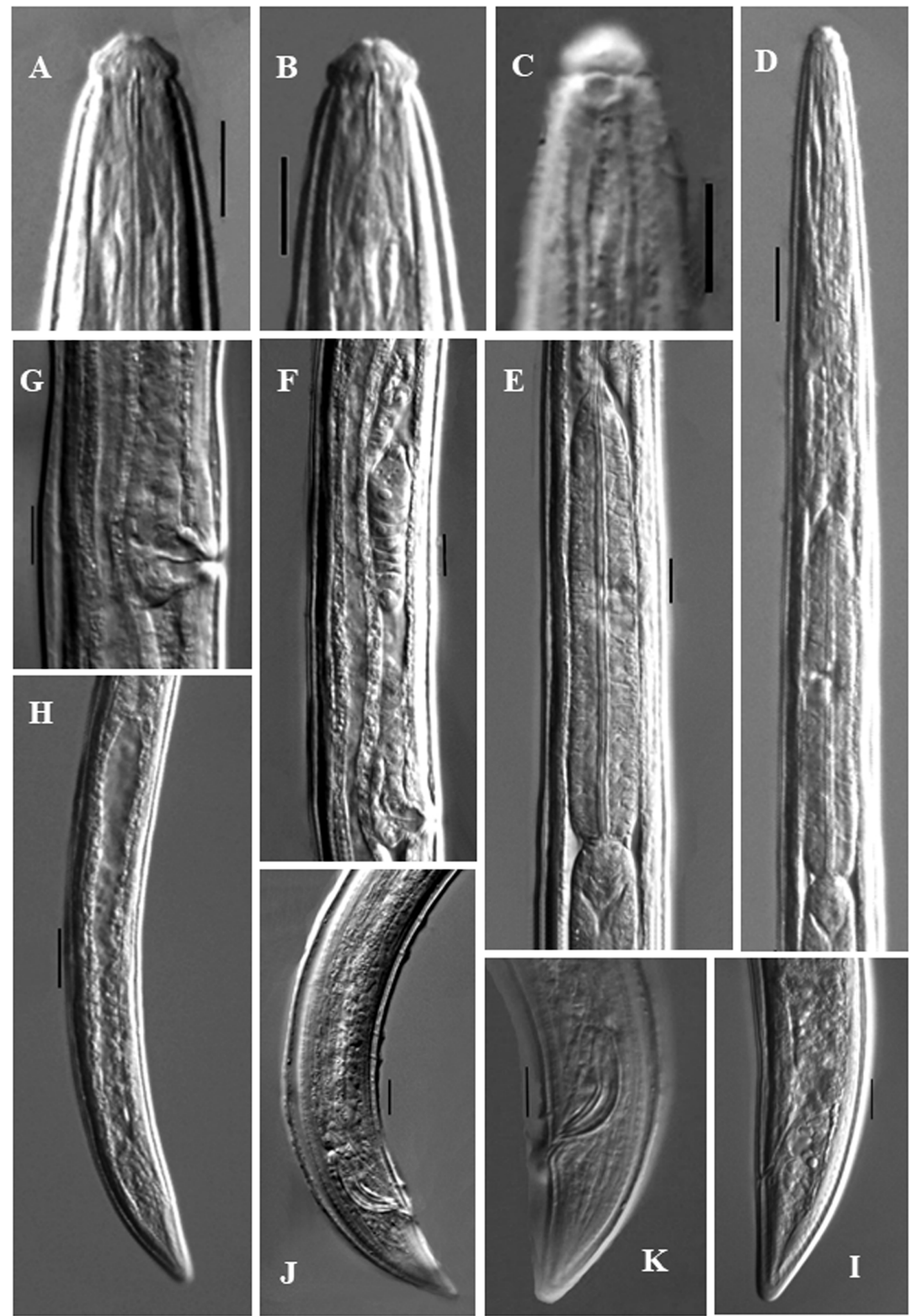

Fig. 2. Tylencholaimus mirabilis (Bütschli, 1873) (LM photographs). A-B. Female anterior region. C. Female anterior region showing amphid. D. Female pharyngeal region. E. Female expanded part of pharynx. F. Female genital system. G. Vulval region. H. Female posterior region. I. Female posterior end. J-K. Male posterior end. Scale bars: A-C, E-G, I-K $=10 \mu \mathrm{m} ; \mathrm{D}, \mathrm{H}=20 \mu \mathrm{m}$. 
Stoma a truncate cone. Odontostyle $0.7-0.9$ times the lip region diameter long, its aperture about $1 / 4$ to $1 / 3$ of its length. Odontophore rod-like, with distinct basal swelling, 1.2-1.6 times the odontostyle length. Guiding ring simple, refractive, at 0.6-0.8 times the lip region diameter from anterior end. Pharynx consisting of a slender, weakly muscular anterior part, expanding abruptly into a cylindrical basal bulb, with thick-walled lumen, separated by a constriction, occupying about $41-46 \%$ of total neck length. Pharyngeal gland nuclei and their orifices are located as follows: $\mathrm{DO}=59-62, \mathrm{DN}=62-65, \mathrm{DO}-\mathrm{DN}=$ $1.7-3.7, \mathrm{~S} 1 \mathrm{~N} 1=72-76, \mathrm{~S} 1 \mathrm{~N} 2=76-80, \mathrm{~S} 2 \mathrm{~N}=89-91, \mathrm{~S} 2 \mathrm{O}=90-93$. Nerve ring at $34-42 \%$ of neck length from anterior end. Cardia rounded to conoid, about $1 / 3$ to $2 / 5$ of the corresponding body diameter long.

Genital system monodelphic-prodelphic. Ovary reflexed, measuring 46-127 $\mu \mathrm{m}$ long, rarely extending beyond the oviduct-uterus junction $(n=4)$; oocytes arranged in single row except near tip. Oviduct joining the ovary subterminally, measuring 38-91 $\mu \mathrm{m}$, its proximal and distal parts not differentiated. Oviduct-uterus junction marked by weak sphincter. Uterus short and tubular, measuring $28-47 \mu \mathrm{m}$ long. Posterior genital branch reduced to very small sac-like structure, measuring 5.0-8.5 $\mu \mathrm{m}$ long or about $1 / 4$ of midbody diameter. Sperm cell absent. Vagina cylindrical, extending inward, 11-15 $\mu \mathrm{m}$, or about $1 / 3$ to $1 / 2(35-50 \%)$ of midbody diameter; pars proximalis vaginae $6.5-9.5 \times 6.0-8.0 \mu \mathrm{m}$, encircled by circular muscles; pars distalis vaginae $4.0-5.5 \mu \mathrm{m}$ with slightly curved walls; pars refringens absent. Vulva apparently a transverse slit. Prerectum 5.6-8.8 and rectum 1.0-1.5 anal body diameter long. Tail convex-conoid with bluntly rounded terminus, $1.2-1.8$ times anal body diameter long, with a pair of caudal pores on each side.

\section{Male}

General morphology similar to that of female, except for posterior region being more ventrally curved. Genital system diorchic, testes opposed, sperm cell spindle-shaped. In addition to adcloacal pair at $7.0-8.0 \mu \mathrm{m}$ from cloacal aperture, there are four ventromedian supplements, located outside the range of spicules, first ventromedian supplement at 37-38 $\mu \mathrm{m}$ from adcloacal pair, second at 7.0-8.0 $\mu \mathrm{m}$ from first, third at $22-23 \mu \mathrm{m}$ from second and fourth at 10-11 $\mu \mathrm{m}$ from third ventromedian supplement. Spicules typically dorylaimoid, curved ventrad, relatively robust, 4.0-4.4 times as long as wide and 1.21.3 times as long as body diameter at level of cloacal aperture, dorsal contour regularly convex, ventral contour bearing a moderately developed hump and hollow, curvature $123-125^{\circ}$, head occupying about $22 \%$ of total spicules length, median pieces $9.5-10.2$ times as long as wide, occupying about $30 \%$ of the spicules maximum width, reaching the spicules tip, posterior end 3.0-3.5 $\mu \mathrm{m}$ wide. Lateral guiding pieces distinct, rod-like, 3.4-4.0 times as long as wide or $1 / 4$ of the spicules length. Prerectum $6.0-7.0$ and rectum 1.2-1.5 times cloacal body diameter long. Tail short, convex-conoid, with bluntly rounded terminus, 1.4 cloacal body diameter in length, with a pair of caudal pores on each side.

\section{Remarks}

Bütschli (1873) described Tylenchus mirabilis from Germany for which de Man (1876) proposed the genus Tylencholaimus with T. mirabilis as its type species. The type specimens were not preserved and the identity of the species remained uncertain. De Man $(1880,1884)$ collected specimens from soil near Apeldoorn, The Netherland in July, 1879 and described them as Tylencholaimus mirabilis. Tarjan (1953) described Discomyctus brevicaudatus from Rhode Island, United States, which he later (Tarjan 1956) transferred to Tylecholaimus. Coomans (1962) redescribed T. brevicaudatus from Belgium and considered it closely similar to T. mirabilis (Bütschli, 1873). Loof \& Jairajpuri (1968) in their revision of the genus Tylencholaimus, redescribed this species based on seven females from the type locality and one male from Switzerland, and designated neotype as well as topotype from these specimens. They also agreed with de Man's (1876) opinion that T. mirabilis is the type species of the genus Tylencholaimus. Vinciguerra (1986) described this species from Italy, while Peña-Santiago \& Coomans (1994b), in their revision of the genus Tylencholaimus, made a detailed study of the type population and of another 
population collected from Spain. The morphometrics of the present specimens collected from India conform well with the type population, except in having slightly smaller spicules ( $27 \mathrm{vs} 31 \mu \mathrm{m})$. The present specimens also conform well with populations described earlier by Tarjan (1953), Loof (1961) and Coomans (1962), except for the presence of males (vs absent). The present specimens also conform with Spanish and Italian populations described by Peña-Santiago \& Coomans (1994b) and Vinciguerra (1986). This is the first report of T. mirabilis from India.

Tylencholaimus teres Thorne, 1939

Fig. 3, Table 2

Tylencholaimus teres Thorne, 1939: 58-59.

Tylencholaimus teres - Jairajpuri 1965: 512. — Thorne 1974: 83. — Vinciguerra 1986: 112. — PeñaSantiago \& Coomans 1994a: 59-66.

\section{Material examined}

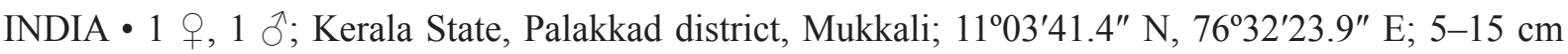
depth; 25 Oct. 2017; soil samples collected from around roots of shrubs (unidentified); slide reference number AMU/ZD/NC/Tylencholaimus teres/1.

\section{Remarks}

Thorne (1939) described Tylencholaimus teres from the USA. Later, Jairajpuri (1965), Vinciguerra (1986) and Thorne (1974) redescribed this species from India, Italy and the USA, respectively. PeñaSantiago \& Coomans (1994a), in their revision of the genus Tylencholaimus, restudied the type material and added two more populations from Spain. The morphometrics of the present specimens conform well with the type population except in having a slightly shorter body length $(0.78 \mathrm{vs} 0.88-1.0 \mathrm{~mm})$; shorter pharyngeal expansion ( $76 \mathrm{vs} 102-110 \mu \mathrm{m})$; slightly anterior vulva position ( $\mathrm{V}=55.6 \mathrm{vs} 58.8-62.3)$; lower c (44.7 vs 55-67) ratio and smaller spicules ( 28 vs $30-32 \mu \mathrm{m})$. The present specimens also conform well with the Indian population except in having a slightly shorter and slender body $(0.78 \mathrm{vs} 0.9 \mathrm{~mm}, \mathrm{a}=30.9$ vs 25 ) and lower c (44.7 vs 50) ratio. The present specimens also conform well with Spanish populations except for having a slightly smaller pharynx (184 vs 191-228 $\mu \mathrm{m})$ and comparatively anterior vulva position $(\mathrm{V}=55.6$ vs $62-66)$. These differences are considered here as intraspecific variability.

Tylencholaimus micronanus Yeates, 1979

Fig. 4, Table 3

Tylencholaimus micronanus Yeates, 1979: 423-425.

Tylencholaimus vanguimus Mohilal \& Dhanachand, 2000: 33-35.

Tylencholaimus micronanus - Peña-Santiago \& Coomans 1994c: 362-364. — Peña-Santiago 2008: 123.

\section{Material examined}

INDIA - Kerala State - 6 o $\odot$; Kasaragod district, Ranipuram National Park; 12.4 $26^{\prime} 18.3^{\prime \prime}$ N, 75.3 $3^{\circ} 8^{\prime} 94.4^{\prime \prime}$ E; 5-15 cm depth; 15 Nov. 2016; soil samples collected from around the roots of grasses (unidentified); slides reference number AMU/ZD/NC/Tylencholaimus micronanus/1-3. - Karnataka

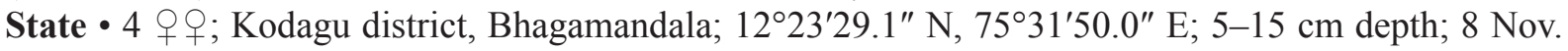
2016; soil samples collected from around the roots of grasses (unidentified); slides reference number AMU/ZD/NC/Tylencholaimus micronanus/4-6. 

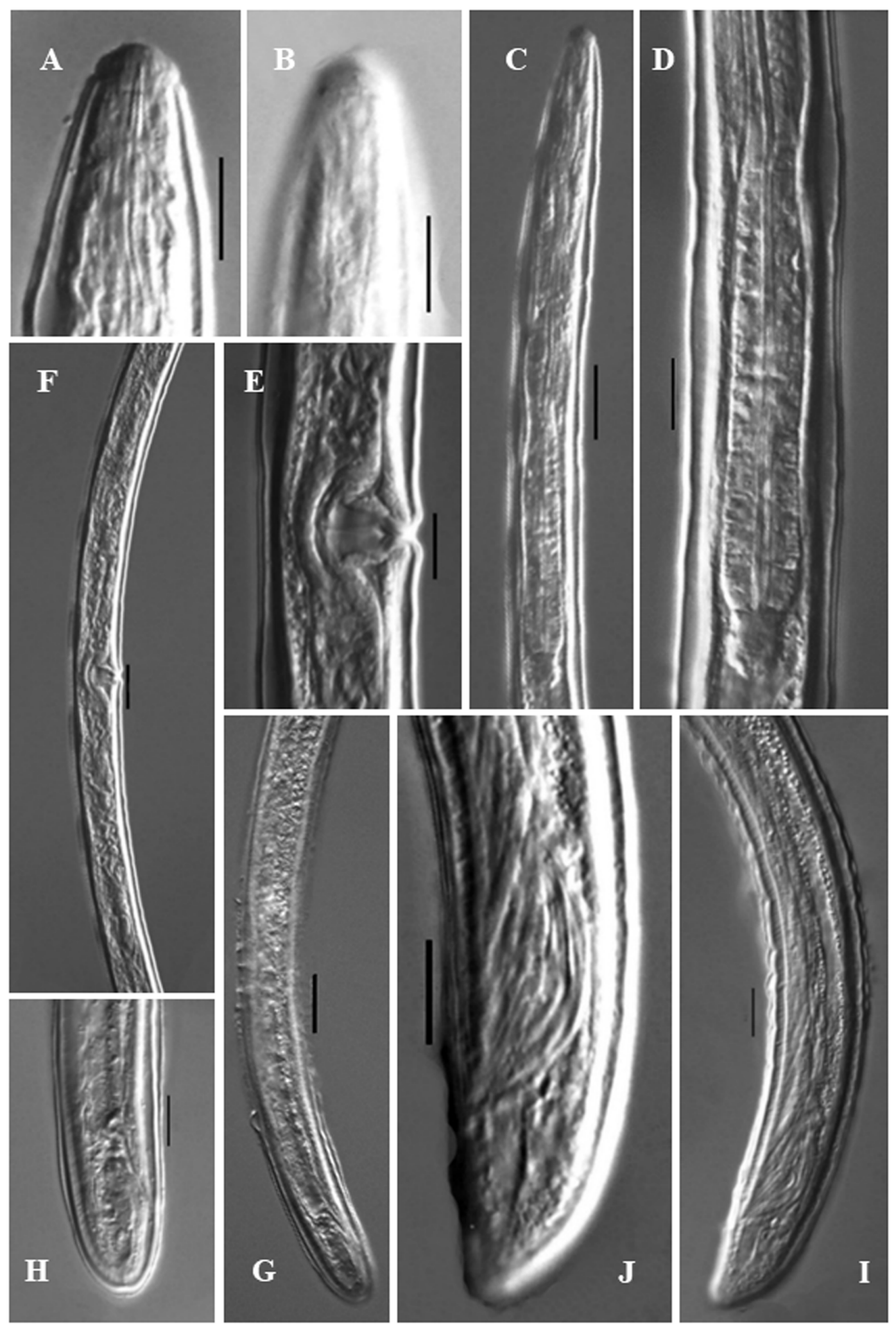

Fig. 3. Tylencholaimus teres Thorne, 1939 (LM photographs). A. Female anterior region. B. Female anterior region showing amphid. C. Male pharyngeal region. D. Male expanded part of pharynx. E. Vulval region. F. Female genital system. G. Female posterior region. H. Female posterior end. I. Male posterior region. J. Male posterior end. Scale bars: A-B, D-E, H-J $=10 \mu \mathrm{m} ; \mathrm{C}, \mathrm{F}-\mathrm{G}=20 \mu \mathrm{m}$. 
Table 2. Measurements of Tylencholaimus teres Thorne, 1939. All measurements are in $\mu \mathrm{m}$.

\begin{tabular}{|c|c|c|}
\hline Characters & Female & Male \\
\hline $\mathrm{n}$ & 1 & 1 \\
\hline $\mathrm{L}$ & 788 & 764 \\
\hline $\mathrm{a}$ & 30.9 & 33.0 \\
\hline $\mathrm{b}$ & 4.2 & 4.5 \\
\hline $\mathrm{c}$ & 44.7 & 46.5 \\
\hline$c^{\prime}$ & 0.9 & 0.9 \\
\hline $\mathrm{V}$ & 55.6 & - \\
\hline G1 & 17.3 & - \\
\hline G2 & 16.3 & - \\
\hline Body diameter at neck base & 24.5 & 22.5 \\
\hline Body diameter at mid body & 26 & 23.5 \\
\hline Body diameter at anus & 19.5 & 17.5 \\
\hline Lip region diameter & 8.0 & 8.0 \\
\hline Lip region height & 4.0 & 4.0 \\
\hline Amphidial aperture & 4.0 & 4.0 \\
\hline Odontostyle length & 7.0 & 7.0 \\
\hline Odontophore length & 8.0 & 8.0 \\
\hline Total stylet length & 15 & 15 \\
\hline Guiding ring from anterior end & 5.0 & 5.0 \\
\hline Nerve ring from anterior end & 69 & 68 \\
\hline Neck length & 184 & 173 \\
\hline Expanded part of pharynx & 76 & 70 \\
\hline Cardia length & 7.0 & 10 \\
\hline Anterior genital branch & 137 & - \\
\hline Posterior genital branch & 129 & - \\
\hline Vaginal length & 15 & - \\
\hline Vulva from anterior end & 439 & - \\
\hline Prerectum length & 95 & 105 \\
\hline Rectum length & 22.5 & 31 \\
\hline Tail length & 17.5 & 16.5 \\
\hline Spicules length & - & 28 \\
\hline Lateral guiding pieces & - & 8.0 \\
\hline Ventromedian supplements & - & 3 \\
\hline
\end{tabular}



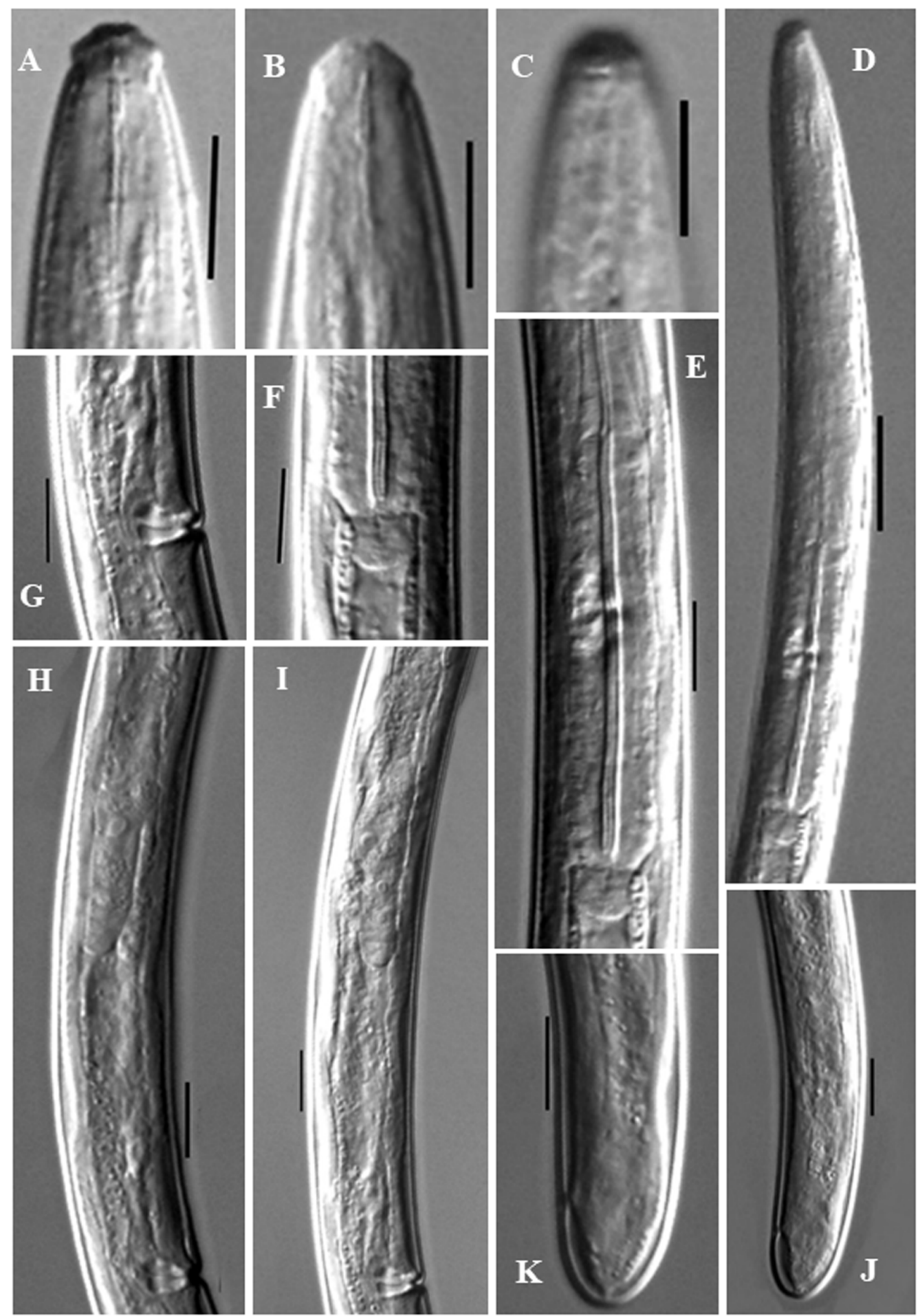

Fig. 4. Tylencholaimus micronanus Yeates, 1979, $q$ (LM photographs). A-B. Anterior region. C. Anterior region showing amphid. D. Pharyngeal region. E. Expanded part of pharynx. F. Pharyngo-intestinal junction. G. Vulval region. H-I. Genital system. J. Posterior region. K. Posterior end. Scale bars: A-C, $\mathrm{E}-\mathrm{K}=10 \mu \mathrm{m} ; \mathrm{D}=20 \mu \mathrm{m}$. 
Table 3. Measurements of Tylencholaimus micronanus Yeates, 1979. All measurements are in $\mu \mathrm{m}$ and in the form: mean \pm s.d. (range).

\begin{tabular}{|c|c|c|}
\hline Localities & Ranipuram population & Bhagamandala population \\
\hline Characters & Females & Females \\
\hline $\mathrm{n}$ & 6 & 4 \\
\hline $\mathrm{L}$ & $348.2 \pm 20.2(312-371)$ & $366.5 \pm 22.6(336-392)$ \\
\hline $\mathrm{a}$ & $18.8 \pm 1.4(17.7-21.2)$ & $22.3 \pm 0.80(21.4-23.5)$ \\
\hline $\mathrm{b}$ & $2.5 \pm 0.11(2.4-2.7)$ & $2.6 \pm 0.13(2.5-2.8)$ \\
\hline $\mathrm{c}$ & $32.1 \pm 3.1(27.5-37.6)$ & $32.8 \pm 0.63(32.6-34.3)$ \\
\hline$c^{\prime}$ & $0.90 \pm 0.07(0.83-1.0)$ & $0.93 \pm 0.03(0.90-1.0)$ \\
\hline $\mathrm{V}$ & $72.6 \pm 1.7(71.1-77.0)$ & $75.1 \pm 0.29(74.4-75.5)$ \\
\hline G1 & $24.0 \pm 2.1(21.1-27.6)$ & $21.6 \pm 0.71(21.0-22.5)$ \\
\hline Body diameter at neck base & $16.7 \pm 0.81(15-18)$ & $16.4 \pm 0.51(15.5-17.5)$ \\
\hline Body diameter at mid body & $17.5 \pm 1.3(15-20)$ & $16.9 \pm 0.81(15.5-18.0)$ \\
\hline Body diameter at anus & $11.5 \pm 0.58(11-13)$ & $11.7 \pm 0.98(11-13)$ \\
\hline Lip region diameter & 6.0 & $5.7 \pm 0.21(5.5-6.0)$ \\
\hline Lip region height & $3.3 \pm 0.18(3.0-3.5)$ & $2.8 \pm 0.21(2.5-3.0)$ \\
\hline Amphidial aperture & $2.6 \pm 0.36(2.0-3.0)$ & $2.2 \pm 0.24(2.0-2.5)$ \\
\hline Odontostyle length & $5.2 \pm 0.23(5.0-5.5)$ & $4.7 \pm 0.21(4.5-5.0)$ \\
\hline Odontophore length & $5.3 \pm 0.28(5.0-6.0)$ & $5.3 \pm 0.34(5.0-6.0)$ \\
\hline Total stylet length & $10.4 \pm 0.36(10-11)$ & $10.1 \pm 0.53(9.5-11)$ \\
\hline Guiding ring from anterior end & 4.0 & $3.7 \pm 0.21(3.5-4.0)$ \\
\hline Nerve ring from anterior end & $57.4 \pm 2.2(54-61)$ & $58.8 \pm 3.6(54-63)$ \\
\hline Neck length & $135.5 \pm 4.2(129-142)$ & $135.9 \pm 2.5(132-139)$ \\
\hline Expanded part of pharynx & $54.3 \pm 3.2(49-57)$ & $52.4 \pm 1.0(51-53)$ \\
\hline Cardia length & $4.9 \pm 0.80(4.0-6.0)$ & $6.1 \pm 0.81(5.0-7.0)$ \\
\hline Anterior genital branch & $85.2 \pm 7.4(78-97)$ & $80.8 \pm 4.6(76-88)$ \\
\hline Vaginal length & $8.6 \pm 0.36(8.0-9.0)$ & $8.2 \pm 0.42(8.0-9.0)$ \\
\hline Vulva from anterior end & $258.3 \pm 17.8(222-278)$ & $275.3 \pm 16.0(253-294)$ \\
\hline Prerectum length & $38.5 \pm 4.8(33-46)$ & $32.8 \pm 0.47(24-43)$ \\
\hline Rectum length & $12.5 \pm 1.0(11-15)$ & $12.0 \pm 1.0(11-14)$ \\
\hline Tail length & $11.5 \pm 0.36(11-12)$ & $11.0 \pm 0.81(10-12)$ \\
\hline
\end{tabular}

\section{Remarks}

Yeates (1979) described Tylencholaimus micronanus from New Zealand. Baqri (1991) reported it from Sikkim, India whereas, Peña-Santiago \& Coomans (1994c) restudied the type material of this species and provided a complete description. Mohilal \& Dhanachand (2000) described a new species, Tylencholaimus vanguimus from Manipur, India which was synonymized with T. micronanus by PeñaSantiago (2008). The morphometrics of the present populations conform well with the type population 
except in having a slightly shorter odontostyle (4.5-5.5 vs 6.0-7.0 $\mu \mathrm{m})$ and in the presence of a terminal caudal pore (vs absent). The present population also conforms well with Sikkim as well as Manipur populations except in having a shorter and robust body $(0.31-0.39$ vs $0.44 \mathrm{~mm}, \mathrm{a}=17-23$ vs 25$)$; lower b value (2.4-2.8 vs 3.6); a slightly shorter odontostyle (4.5-5.5 vs $6.0 \mu \mathrm{m})$ and tail (11-12 vs 13 $\mu \mathrm{m})$ than in the Sikkim population; and a slightly lower c' ratio (0.8-1.0 vs 1.0-1.1) than in Manipur population, as well as the presence of terminal caudal pore (vs absent in the Manipur population).

Tylencholaimus ibericus Peña-Santiago \& Coomans, 1994

Fig. 5, Table 4

Tylencholaimus ibericus Peña-Santiago \& Coomans, 1994c: 355-358.

Tylencholaimus japonicus Ahmad \& Araki, 2003: 9-12.

Tylencholaimus zhongshanensis Wu et al., 2019: 4-8.

Tylencholaimus ibericus - Dhanam \& Jairajpuri 1999: 3.—Ahad \& Ahmad 2016: 364-466.

Tylencholaimus japonicus - Li et al. 2008: 2000.

\section{Material examined}

INDIA - Kerala State - 2 qq, 1 ó; Thiruvananthapuram district, Ponmudi hill; 8 $8^{\circ} 45^{\prime} 36.3^{\prime \prime}$ N, $77^{\circ} 07^{\prime} 08.3^{\prime \prime}$ E; 5-15 cm depth; 4 Nov. 2017; soil samples collected from around the roots of grasses and shrubs (unidentified); slides reference number AMU/ZD/NC/Tylencholaimus ibericus/1-2. Karnataka State - 3 우; Kodagu district, Bhagamandala; $12^{\circ} 23^{\prime 2} 29.1^{\prime \prime}$ N, 75³1'50.0" E; 5-15 cm depth; 8 Nov. 2016; soil samples collected from around the roots of forest plants (unidentified); slides reference number AMU/ZD/NC/Tylencholaimus ibericus/3-4.

\section{Description}

Female

Slender nematodes of small size, slightly curved ventrad upon fixation; body cylindrical, tapering gradually towards both extremities. Cuticle with two distinct layers, 1.5-2.0 $\mu \mathrm{m}$ thick at midbody and 2.0-2.5 $\mu \mathrm{m}$ on tail. Outer cuticle finely striated; inner layer thick, loose, its outline irregular with distinct radial refractive elements. Lateral chords occupying about $23-28 \%$ of midbody diameter. Lateral, dorsal and ventral body pores indistinct. Lip region cap-like, offset by constriction, 1.9-2.3 times as wide as high or about $1 / 3$ to $2 / 5$ of the body diameter at neck base. Lips rounded, amalgamated, inner part slightly elevated. Amphids cup-shaped, their aperture occupying about $1 / 3$ to $2 / 5$ of lip region diameter. Stoma a truncate cone. Odontostyle $0.7-0.9$ times the lip region diameter long, its aperture about $1 / 4$ to $1 / 3$ of the odontostyle length. Odontophore rod-like, with minute basal knobs, 1.0-1.1 times the odontostyle length. Guiding ring simple, refractive, at 0.5-0.6 times the lip region diameter from anterior end. Pharynx consisting of a slender, slightly muscular anterior part, expanding gradually into a cylindrical basal bulb, with thick-walled lumen, occupying about 37-39\% of total neck length. Pharyngeal gland nuclei and their orifices are located as follows: $\mathrm{DO}=62-66, \mathrm{DN}=65-69, \mathrm{DO}-\mathrm{DN}=1.8-2.9, \mathrm{~S} 1 \mathrm{~N} 1=$ $75-79, \mathrm{~S} 1 \mathrm{~N} 2=79-81, \mathrm{~S} 2 \mathrm{~N}=88-91, \mathrm{~S} 2 \mathrm{O}=90-92$. Nerve ring at $37-42 \%$ of neck length from anterior region. Cardia rounded to conoid, about $1 / 4$ to $2 / 5$ of the corresponding body diameter long.

Genital system monodelphic-prodelphic. Ovary reflexed, measuring 30-49 $\mu \mathrm{m}$ long; oocytes arranged in single row except near tip. Oviduct joining the ovary subterminally, measuring $28-57 \mu \mathrm{m}$, consisting of a slender portion and a slightly developed pars dilatata. Oviduct-uterus junction marked with weak sphincter. Uterus short and tubular, measuring 15-31 $\mu \mathrm{m}$. Posterior genital branch completely absent. Vagina slightly anteriorly directed, $8.5-10 \mu \mathrm{m}$ or about $1 \frac{1}{2}(48-55 \%)$ of midbody diameter; pars proximalis vaginae $5.0-6.5 \times 2.5-3.5 \mu \mathrm{m}$, encircled by circular muscles; pars distalis vaginae $3.0-3.5 \mu \mathrm{m}$ with slightly curved walls; pars refringens absent. Vulva apparently a transverse slit. Prerectum 3.3-4.9 and 

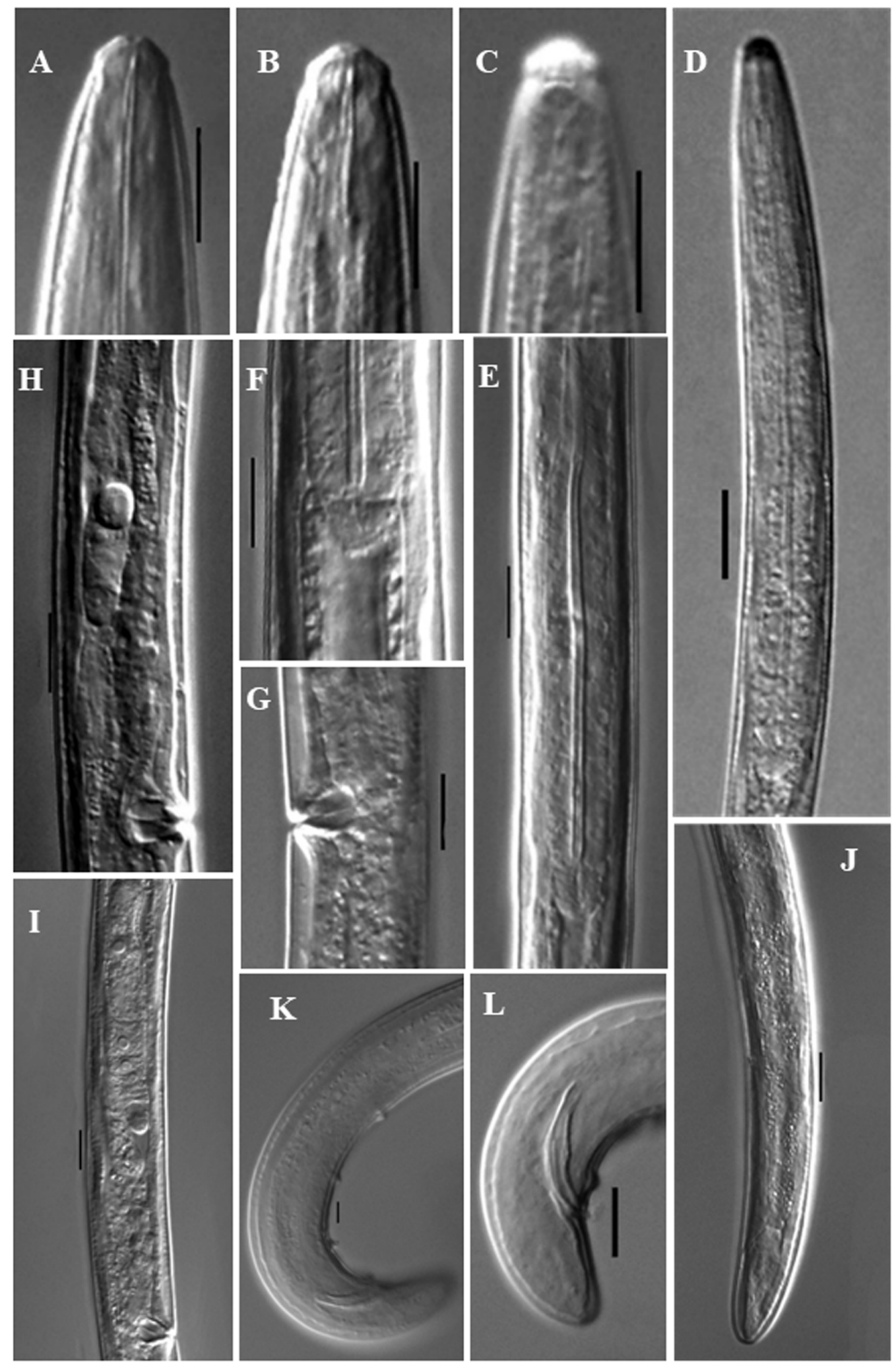

Fig. 5. Tylencholaimus ibericus Peña-Santiago \& Coomans, 1994 (LM photographs). A-B. Female anterior region. C. Female anterior region showing amphid. D. Female pharyngeal region. E. Female expanded part of pharynx. F. Female pharyngo-intestinal junction. G. Vulval region. H-I. Female genital system. J. Female posterior region. K. Male posterior region. L. Male posterior end. Scale bars: $\mathrm{A}-\mathrm{C}, \mathrm{E}-\mathrm{L}=10 \mu \mathrm{m} ; \mathrm{D}=20 \mu \mathrm{m}$. 
Table 4. Measurements of Tylencholaimus ibericus Peña-Santiago \& Coomans, 1994. All measurements are in $\mu \mathrm{m}$ and in the form: mean \pm s.d. range) for the Bhagamandala population.

\begin{tabular}{|c|c|c|c|}
\hline \multirow{2}{*}{$\begin{array}{l}\text { Localities } \\
\text { Characters }\end{array}$} & \multicolumn{2}{|c|}{ Thrivananthapuram population } & \multirow{2}{*}{$\begin{array}{c}\text { Bhagamandala population } \\
\text { Females }\end{array}$} \\
\hline & Females & Male & \\
\hline $\mathrm{n}$ & 2 & 1 & 3 \\
\hline $\mathrm{L}$ & 491,581 & 431 & $474.9 \pm 15.7(460-496)$ \\
\hline $\mathrm{a}$ & 25,28 & 20.5 & $26.9 \pm 1.62(26-29)$ \\
\hline $\mathrm{b}$ & $3.0,3.6$ & 3.1 & $3.1 \pm 0.17(3.0-3.4)$ \\
\hline $\mathrm{c}$ & 32,33 & 28.7 & $29.5 \pm 3.8(24.7-33.8)$ \\
\hline$c^{\prime}$ & $1.0,1.1$ & 0.93 & $1.2 \pm 0.14(1.1-1.5)$ \\
\hline $\mathrm{V}$ & 70,71 & - & $69.8 \pm 0.51(69.6-70.8)$ \\
\hline G1 & 14,16 & - & $15.1 \pm 1.1(13.7-16.6)$ \\
\hline Body diameter at neck base & 20,21 & 20.5 & $18.2 \pm 1.66(16-20)$ \\
\hline Body diameter at mid body & 19,20 & 21 & $17.3 \pm 1.2(15-18)$ \\
\hline Body diameter at anus & 13,15 & 14 & $12.8 \pm 0.23(12.5-13.0)$ \\
\hline Lip region diameter & $7.0,7.0$ & 7.0 & $6.6 \pm 0.23(6.5-7.0)$ \\
\hline Lip region height & $3.0,3.0$ & 3.0 & $3.2 \pm 0.23(3.0-3.5)$ \\
\hline Amphidial aperture & $2.5,3.0$ & 3.0 & $2.5 \pm 0.40(2.0-3.0)$ \\
\hline Odontostyle length & $5.5,5.5$ & 5.5 & $5.5 \pm 0.46(5.0-6.0)$ \\
\hline Odontophore length & $5.5,6.5$ & 5.5 & $5.7 \pm 0.23(5.5-6.0)$ \\
\hline Total stylet length & 11,12 & 11 & $11.27 \pm 0.69(10.5-12)$ \\
\hline Guiding ring from anterior end & $4.0,4.0$ & 4.0 & $3.7 \pm 0.23(3.5-4.0)$ \\
\hline Nerve ring from anterior end & 60,65 & 58 & $60.0 \pm 1.2(58-61)$ \\
\hline Neck length & 156,160 & 137 & $148.6 \pm 10.1(134-156)$ \\
\hline Expanded part of pharynx & 62,63 & 51 & $58.1 \pm 6.5(52-62)$ \\
\hline Cardia length & $5.0,5.5$ & 7 & $7.1 \pm 1.22(5.0-8.0)$ \\
\hline Anterior genital branch & 81,84 & - & $75.5 \pm 7.71(64-82)$ \\
\hline Vaginal length & $9.5,9.0$ & - & $9.1 \pm 0.4(8.5-10.0)$ \\
\hline Vulva from anterior end & 347,411 & - & $333.2 \pm 9.7(322-345)$ \\
\hline Prerectum length & 49,53 & 78 & $49.6 \pm 5.32(42-53)$ \\
\hline Rectum length & 11,16 & 22 & $13.1 \pm 1.6(10-14)$ \\
\hline Tail length & 14,17 & 15 & $16.0 \pm 1.8(14-18)$ \\
\hline Spicules length & - & 20 & - \\
\hline Lateral guiding pieces & - & 5.0 & - \\
\hline Ventromedian supplements & - & 3 & - \\
\hline
\end{tabular}


rectum $0.8-1.1$ times the anal body diameter long. Tail short, rounded to conoid, $1.0-1.5$ times the anal body diameter long, with a pair of subdorsal and a prominent terminal caudal pore.

\section{Male}

General morphology similar to that of female, except for posterior region being more ventrally curved. Genital system diorchic, testes opposed, sperm cell spindle-shaped. In addition to the adcloacal pair at $6.0 \mu \mathrm{m}$ from cloacal aperture, there are three ventromedian supplements, located outside the range of spicules, first one at $12 \mu \mathrm{m}$ from adcloacal pair, second at $16 \mu \mathrm{m}$ from first and third one at $18 \mu \mathrm{m}$ from second ventromedian supplement. Spicules typically dorylaimoid, curved ventrad, relatively slender, 5.7 times as long as wide and 1.4 times cloacal body diameter long, dorsal contour regularly convex, ventral contour bearing a moderately developed hump and hollow, curvature $140^{\circ}$, head occupying about $25 \%$ of total spicules length, median piece 10.6 times as long as wide, occupying about $42 \%$ of the spicules maximum width, reaching the spicules tip, posterior end $2.5 \mu \mathrm{m}$ wide. Lateral guiding piece distinct, rod-like, about 5.0 times as long as wide or about $1 / 5$ of the spicules length. Prerectum 5.7 and rectum 1.5 times the cloacal body diameter long. Tail short, rounded to conoid, 0.93 times the cloacal body diameter long, with a pair of caudal pores on each side.

\section{Remarks}

Peña-Santiago \& Coomans (1994c) described Tylencholaimus ibericus from Spain. Dhanam \& Jairajpuri (1999) reported this species from Karnataka, India. Ahmad \& Araki (2003) described Tylencholaimus japonicus from Japan, which was considered a synonym of T. ibericus by Peña-Santiago (2008). Later, Li et al. (2008) and Ahad \& Ahmad (2016) reported it from China. Wu et al. (2019) described Tylencholaimus zhongshanensis from Zhongshan, China. Recently, Peña-Santiago (2020) synonymized this species with $T$. ibericus that was accepted herein as well. The morphometrics of the present populations conform well with the type population except in having distinct radial refractive elements (vs indistinct); slightly shorter odontophore (5.5-6.5 vs 6.0-8.0 $\mu \mathrm{m})$ and presence of male (vs male absent). The present populations conform well with the Indian population described by Dhanam \& Jairajpuri (1999) except in having slightly lower b (3.0-3.6 vs 4.0) ratio; shorter tail length (14-18 vs $18-20 \mu \mathrm{m}$ ) and presence of male (vs absent). The morphometrics of the present populations also conform well with the Japanese population described by Ahmad \& Araki (2003) except in having lower c (24-33 vs $35-46)$ and slightly higher c' (1.0-1.5 vs $0.92-1.1)$ ratios; shorter prerectum (42-53 vs $70-112 \mu \mathrm{m})$ and presence of male (vs absent). The present populations also conform well with earlier as well as recently described Chinese populations by Li et al. (2008), Ahad \& Ahmad (2016) and Wu et al. (2019). These differences may be interpreted as geographical or intraspecific variability. Male individuals is reported here for the first time in this species.

Tylencholaimus cosmos (Dhanam \& Jairajpuri, 1999)

Fig. 6 , Table 5

Amphitylencholaimus cosmos Dhanam \& Jairajpuri, 1999: 2, 4

Tylencholaimus cosmos - Peña-Santiago 2008: 120-121.

\section{Material examined}

INDIA - Goa State • 3 q 9 ; South Goa district, Verna; $15^{\circ} 35.3^{\prime} 59.3^{\prime \prime}$ N, $73^{\circ} 92.2^{\prime} 26.4^{\prime \prime}$ E; 5-15 cm depth; 22 Apr. 2016; soil sample collected from around the roots of grasses (unidentified); slide reference number AMU/ZD/NC/Tylencholaimus cosmos/1. - Kerala State • 3 우; Thiruvananthapuram district, Ponmudi hill; 8 $45^{\prime} 36.2^{\prime \prime} \mathrm{N}, 77^{\circ} 07^{\prime} 08.2^{\prime \prime}$ E; 5-15 cm depth; 4 Nov. 2017; soil sample collected from around the roots of grasses (unidentified); slide reference number AMU/ZD/NC/Tylencholaimus cosmos/2. 

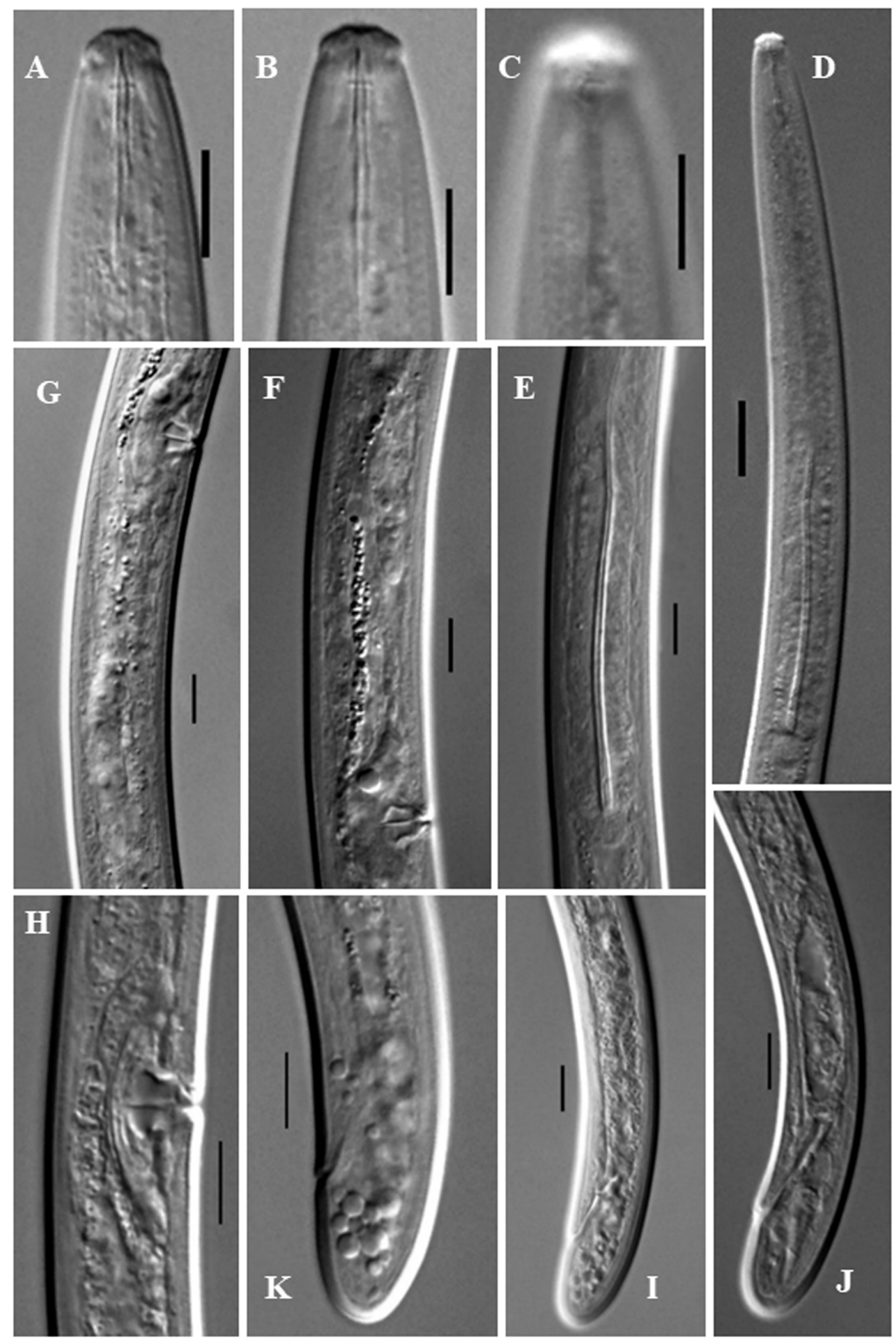

Fig. 6. Tylencholaimus cosmos (Dhanam \& Jairajpuri, 1999), $q$ (LM photographs). A-B. Anterior region. C. Anterior region showing amphid. D. Pharyngeal region. E. Expanded part of pharynx. F-G. Genital system. H. Vulval region. I-J. Posterior region. K. Posterior end. Scale bars: A-C, E-K $=10 \mu \mathrm{m}$; D = $20 \mu \mathrm{m}$. 
Table 5. Measurements of Tylencholaimus cosmos (Dhanam \& Jairajpuri, 1999). All measurements are in $\mu \mathrm{m}$ and in the form: mean \pm s.d. (range).

\begin{tabular}{|c|c|c|}
\hline Localities & Verna population & Ponmudi population \\
\hline Characters & Females & Females \\
\hline $\mathrm{n}$ & 3 & 3 \\
\hline $\mathrm{L}$ & $645.8 \pm 21.5(616-667)$ & $656.9 \pm 86.6(555-767)$ \\
\hline $\mathrm{a}$ & $28.9 \pm 1.8(27.3-31.7)$ & $29.8 \pm 3.3(25.7-33.0)$ \\
\hline $\mathrm{b}$ & $3.9 \pm 0.13(3.8-4.2)$ & $3.5 \pm 0.39(3.1-4.1)$ \\
\hline $\mathrm{c}$ & $37.3 \pm 0.38(37.0-37.8)$ & $34.5 \pm 3.9(29.8-39.1)$ \\
\hline$c^{\prime}$ & $1.03 \pm 0.03(1.0-1.1)$ & 1.1 \\
\hline $\mathrm{V}$ & $58.8 \pm 0.92(58.5-60.5)$ & $60.4 \pm 0.92(59.6-61.7)$ \\
\hline G1 & $13.1 \pm 0.42(12.6-13.6)$ & $12.7 \pm 1.0(11.8-14.2)$ \\
\hline G2 & $11.2 \pm 1.0(10.2-12.6)$ & $11.7 \pm 0.65(11.0-12.5)$ \\
\hline Body diameter at neck base & $21.2 \pm 0.92(20-22)$ & $20.5 \pm 1.6(18-22)$ \\
\hline Body diameter at mid body & $21.8 \pm 0.92(20-22)$ & $21.5 \pm 1.6(19-23)$ \\
\hline Body diameter at anus & $15.5 \pm 0.46(15-16)$ & $16.6 \pm 0.80(15-17)$ \\
\hline Lip region diameter & 8.0 & 8.0 \\
\hline Lip region height & $3.7 \pm 0.23(3.0-4.0)$ & $3.1 \pm 0.23(3.0-3.5)$ \\
\hline Amphidial aperture & 2.0 & $2.6 \pm 0.23(2.5-3.0)$ \\
\hline Odontostyle length & $7.8 \pm 0.23(7.5-8.0)$ & $7.6 \pm 0.23(7.0-8.0)$ \\
\hline Odontophore length & $8.9 \pm 0.23(8.5-9.0)$ & $9.6 \pm 0.23(9.5-10)$ \\
\hline Total stylet length & $16.6 \pm 0.40(16.5-17.0)$ & $17.8 \pm 0.23(17.5-18.0)$ \\
\hline Guiding ring from anterior end & 5.5 & $5.2 \pm 0.23(5.0-5.5)$ \\
\hline Nerve ring from anterior end & $75.1 \pm 5.4(67-80)$ & $70.8 \pm 3.7(65-74)$ \\
\hline Neck length & $160.7 \pm 2.7(158-164)$ & $180.6 \pm 4.5(174-185)$ \\
\hline Expanded part of pharynx & $66.6 \pm 2.1(63-68)$ & $75.7 \pm 0.46(75-76)$ \\
\hline Cardia length & 5.0 & $4.9 \pm 0.80(4.0-5.5)$ \\
\hline Anterior genital branch & $86.5 \pm 4.6(82-93)$ & $85.5 \pm 11.3(69-94)$ \\
\hline Posterior genital branch & $73.8 \pm 4.4(68-79)$ & $78.0 \pm 7.8(71-89)$ \\
\hline Vaginal length & $9.9 \pm 0.23(9.5-10.0)$ & $10.3 \pm 0.46(9.5-10.5)$ \\
\hline Vulva from anterior end & $386.4 \pm 18.4(360-402)$ & $396.2 \pm 47.1(343-457)$ \\
\hline Prerectum length & $37.5 \pm 2.0(35-40)$ & $39.2 \pm 1.3(37-40)$ \\
\hline Rectum length & $19.2 \pm 1.2(17-20)$ & $21.8 \pm 0.46(21-22)$ \\
\hline Tail length & $17.3 \pm 0.46(16.0-17.5)$ & $18.6 \pm 0.80(17-19)$ \\
\hline
\end{tabular}




\section{Remarks}

Dhanam \& Jairajpuri (1999) described Amphitylencholaimus cosmos from Malnad tracts of Karnataka, India. Peña-Santiago (2008) did not accept the generic status of Amphitylencholaimus and transferred A. cosmos to Tylencholaimus. Ahad \& Ahmad (2016) reported this species from Kaziranga National Park, Assam, India. Dhanam \& Jairajpuri (1999) characterized this species by having the anterior slender part of pharynx expanding gradually to form the cylindroid basal part but in their fig. 1C, the anterior part of pharynx appears slender, expanding abruptly to form the basal expanded part. In the present specimens, the anterior part of pharynx is also slender, expanding abruptly to form the basal expanded part. The morphometrics of the present populations conform well with the type population except in having a shorter body length $(0.55-0.76$ vs $0.7-0.9 \mathrm{~mm})$, slightly lower $b$ value (3.1-4.2 vs 3.9-5.0) and shorter odontophore length ( $8.5-10 \mathrm{vs} 12-14 \mu \mathrm{m})$. The present populations also conform well with the Assam population except in having a slightly higher lip region (3.0-3.5 vs $2.0-3.0 \mu \mathrm{m})$ and a longer rectum $(17-22$ vs $13-14 \mu \mathrm{m})$.

Tylencholaimus macroamphidius sp. nov. urn:1sid:zoobank.org:act:10F3DAD5-CF89-4677-95FA-A06D29395E95

Figs 7-8, Table 6

\section{Diagnosis}

Tylencholaimus macroamphidius sp. nov. is characterized by having $0.6-0.8 \mathrm{~mm}$ long, slender body; lip region set off by deep constriction, lips separate, angular; amphids large; odontostyle 8.0-9.0 $\mu \mathrm{m}$, odontophore 8.5-9.5 $\mu \mathrm{m}$ with asymmetrical basal knobs, total stylet length 17.0-18.5 $\mu \mathrm{m}$; pharynx with weakly muscular anterior part expanding gradually into a cylindrical basal bulb occupying about $38-43 \%$ of total neck length; female genital system monodelphic-prodelphic; posterior branch 39$70 \mu \mathrm{m}$ or $2.0-3.5$ times the midbody diameter long, consisting of uterus, sphincter, and with a saclike structure representing a rudimentary oviduct; vulva transverse; tail rounded to conoid and males with 19-23 $\mu \mathrm{m}$ long spicules, lateral guiding pieces 5.0-6.0 $\mu \mathrm{m}$ long and three spaced ventromedian supplements.

\section{Etymology}

The new species is named Tylencholaimus macroamphidius sp. nov. because of its characteristically large amphids.

\section{Material examined}

\section{Holotype}

INDIA • 1 क; Tamil Nadu State, Nilgiris Hill, Naduvattum; $11^{\circ} 28^{\prime} 37.8^{\prime \prime}$ N, 76 $32^{\prime} 36.7^{\prime \prime}$ E; 5-15 cm depth; 15 Nov. 2016; roots of shrubs (unidentified); slide reference number AMU/ZD/NC/Tylencholaimus macroamphidius/1.

\section{Paratypes}

INDIA -4 우, 2 ô; same collection data as for holotype; slides reference number AMU/ZD/NC/

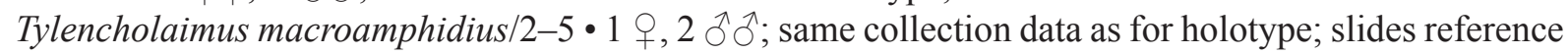
number AMU/ZD/NC/Tylencholaimus macroamphidius/6-8; nematode collection of the Zoological Survey of India, Kolkata, India.

\section{Type habitat and locality}

Soil samples collected from around the roots of shrubs (unidentified) from Naduvattum, Nilgiri Hills, Tamil Nadu State. 

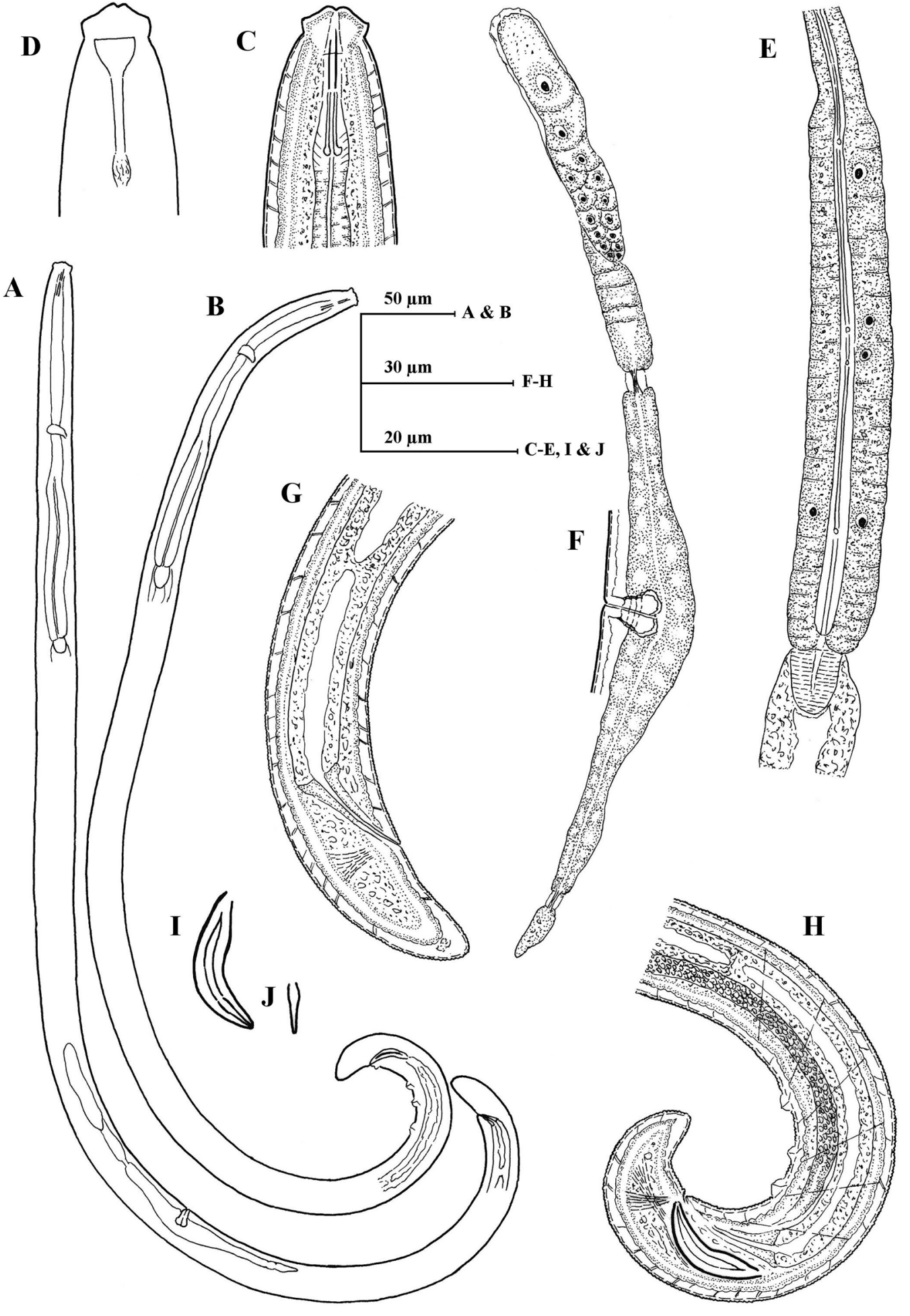

A

Fig. 7. Tylencholaimus macroamphidius sp. nov. A, C, F-G. Holotype, $q$ (AMU/ZD/NC/Tylencholaimus macroamphidius/1). B, D, H-J. Paratype 6, ô (slide 5). E. Paratype 3, + (slide 3). A. Entire female. B. Entire male. C. Female anterior region. D. Male anterior region showing amphid. E. Female expanded part of pharynx. F. Female genital system. G. Female posterior region. H. Male posterior region. I. Spicule. J. Lateral guiding piece. 

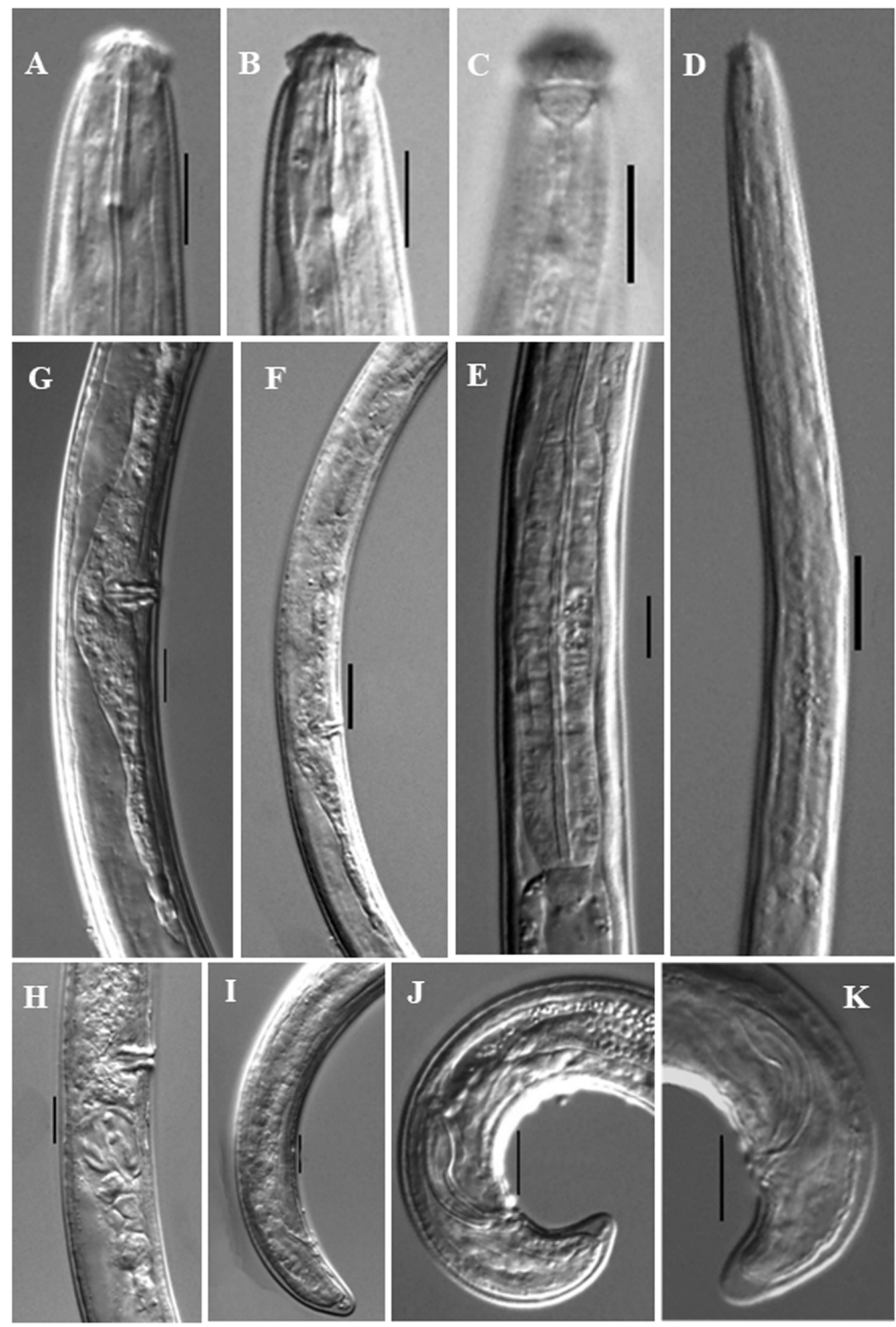

Fig. 8. Tylencholaimus macroamphidius sp. nov. (LM photographs). A-B, F, G, I. Holotype, $\bigcirc$ (AMU/ ZD/NC/Tylencholaimus macroamphidius/1). C. Paratype 6, ồ (slide 5). D-E. Paratype 2, q (slide 3). H. Paratype 1, $q$ (slide 2). J. Paratype 7, ô (slide 6). K. Paratype 9, ô (slide 8). A-B. Female anterior region. C. Male anterior region showing amphid. D. Female pharyngeal region. E. Female expanded part of pharynx. F-H. Female genital system. I. Female posterior region. J. Male posterior region. K. Male posterior end showing spicules. Scale bars: A-C, E, G-K $=10 \mu \mathrm{m} ; \mathrm{D}, \mathrm{F}=20 \mu \mathrm{m}$. 


\section{Description}

Female

Small sized nematodes, slightly curved ventrad upon fixation; body cylindrical, tapering gradually towards both extremities but more so towards the anterior end. Cuticle with two distinct layers, 1.5$2.0 \mu \mathrm{m}$ thick at midbody and $2 \mu \mathrm{m}$ on tail. Outer cuticle thin, finely striated; inner layer thick, its outline somewhat irregular, with distinct radial refractive elements, more distinct in caudal region. Lateral chords occupying about $25-29 \%$ of midbody diameter. Lateral, dorsal and ventral body pores indistinct. Lip region cap-like, offset by deep constriction, 2.0-2.5 times as wide as high or about $1 / 2$ to $2 / 5$ of the body diameter at neck base. Lips moderately separate, angular, inner part slightly elevated and protruding. Labial and cephalic papillae distinct but not interfering with the labial contour. Amphids large, stirrup-shaped, their aperture occupying about $1 / 2$ to $3 / 5$ of lip region diameter. Stoma a truncate cone. Odontostyle $0.8-1.0$ times the lip region diameter long, its aperture about $1 / 3$ of the odontostyle length. Odontophore 1.0-1.1 times of the odontostyle length with asymmetrical basal knobs, the subventral knobs always larger than dorsal one. Guiding ring simple, refractive, at 0.6-0.9 times the lip region diameter from anterior end. Pharynx consisting of a slender, slightly muscular anterior part, expanding gradually into a cylindroid basal bulb, with thick-walled lumen, occupying about $38-43 \%$ of total neck length. Pharyngeal gland nuclei and their orifices are located as follows: DO $=61-64$, $\mathrm{DN}=63-67, \mathrm{DO}-\mathrm{DN}=1.5-3.1, \mathrm{~S} 1 \mathrm{~N} 1=73-76, \mathrm{~S} 1 \mathrm{~N} 2=77-81, \mathrm{~S} 2 \mathrm{~N}=88-90, \mathrm{~S} 2 \mathrm{O}=90-91$. Nerve ring at $33-39 \%$ of neck length from anterior region. Cardia rounded to conoid, about $1 / 3$ to $3 / 5$ of the corresponding body diameter long.

Genital system monodelphic-prodelphic. Ovary reflexed, measuring 24-49 $\mu \mathrm{m}$ long; oocytes arranged in single row except near tip. Oviduct joining the ovary subterminally, measuring 49-84 $\mu \mathrm{m}$, consisting of a slender distal part and a well developed pars dilatata. Oviduct-uterus junction marked with welldeveloped sphincter. Uterus short, tubular, measuring 33-45 $\mu \mathrm{m}$. Posterior genital branch 39-70 $\mu \mathrm{m}$ or 2.0-3.5 times the midbody diameter long, comprised of uterine part measuring 29-54 $\mu \mathrm{m}$ and distinct sphincter followed by sac-like structure representing rudimentary oviduct. Sperm cell present throughout the genital tract. Vagina cylindrical, extending inwards, $8.5-10.5 \mu \mathrm{m}$ or about $2 / 5$ to $1 / 2(43-53 \%)$ of midbody diameter; pars proximalis vaginae $6.5-7.0 \times 2.0-2.5 \mu \mathrm{m}$, encircled by circular muscles; pars distalis vaginae $3.0-3.5 \mu \mathrm{m}$ with slightly curved walls; pars refringens absent. Vulva a transverse slit. Prerectum 2.6-4.0 and rectum 0.9-1.0 times anal body diameter long. Tail short, conoid, ventrally curved with rounded terminus, 1.4-1.6 times anal body diameter long, with a pair of subdorsal caudal pores.

\section{Male}

General morphology similar to that of female, except for the posterior body region being comparatively more ventrally curved. Genital system diorchic, testes opposed, sperm cell spindle-shaped. In addition to adcloacal pair at 3.0-5.0 $\mu \mathrm{m}$ from cloacal aperture, there are three ventromedian supplements, located outside the range of spicules, first one at 16-20 $\mu \mathrm{m}$ from adcloacal pair, second at $8.0-10 \mu \mathrm{m}$ from first, and third at $8.0-14 \mu \mathrm{m}$ from second ventromedian supplement. Spicules typically dorylaimoid, curved ventrad, relatively robust, 4.9-5.8 times as long as wide, 1.0-1.4 times as long as cloacal body diameter, dorsal contour regularly convex, ventral contour bearing a moderately developed hump and hollow, curvature $120-123^{\circ}$, head occupying about $14-17 \%$ of total spicules length, median pieces $11.3-12.5$ times as long as wide, occupying about $37 \%$ of the spicules maximum width, reaching the spicules tip, posterior end 2.0-2.5 $\mu \mathrm{m}$ wide. Lateral guiding pieces slender, about 4.0-6.0 times as long as wide or about $1 / 3$ of total spicules length. Prerectum $4.2-5.0$ and rectum 1.0-1.2 cloacal body diameter long. Tail short, conoid, 1.1-1.2 times cloacal body diameter long with a pair of subdorsal caudal pores.

\section{Taxonomic remarks}

In the presence of asymmetrical basal knobs of the odontophore, the new species comes close to T. confusus Ahmad \& Araki, 2003 and T. arakii Ahad \& Ahmad, 2016, but differs from the former in 
Table 6. Measurements of Tylencholaimus macroamphidius sp. nov. All measurements are in $\mu \mathrm{m}$ and in the form: mean \pm s.d. (range).

\begin{tabular}{|c|c|c|c|}
\hline Characters & Holotype female & Paratypes females & Paratypes males \\
\hline $\mathrm{n}$ & & 5 & 4 \\
\hline $\mathrm{L}$ & 760 & $693.2 \pm 85.3614-850)$ & $681.5 \pm 52.1(604-736)$ \\
\hline $\mathrm{a}$ & 38.8 & $34.6 \pm 3.1(32.5-41.3)$ & $34.2 \pm 2.8(32.0-39.5)$ \\
\hline $\mathrm{b}$ & 3.8 & $3.7 \pm 0.36(3.4-4.3)$ & $3.6 \pm 0.23(3.3-4.0)$ \\
\hline $\mathrm{c}$ & 37.0 & $30.3 \pm 3.5(28.2-37.7)$ & $34.5 \pm 2.7(30.8-37.6)$ \\
\hline$c^{\prime}$ & 1.4 & $1.55 \pm 0.04(1.5-1.6)$ & $1.15 \pm 0.05(1.1-1.2)$ \\
\hline $\mathrm{V}$ & 66.9 & $66.2 \pm 1.0(65.7-68.5)$ & - \\
\hline G1 & 14.6 & $17.6 \pm 2.4(13.5-20.3)$ & - \\
\hline G2 & 9.0 & $7.1 \pm 1.3(6.0-9.7)$ & - \\
\hline Body diameter at neck base & 19 & $29.5 \pm 1.5(26-31)$ & $19.3 \pm 1.4(17-22)$ \\
\hline Body diameter at mid body & 20 & $19.6 \pm 0.87(18-20)$ & $19.6 \pm 1.8(17-23)$ \\
\hline Body diameter at anus & 15 & $14.1 \pm 0.48(13-15)$ & $16.1 \pm 0.84(15-17)$ \\
\hline Lip region diameter & 9.5 & $9.1 \pm 0.4(9.0-10)$ & $9.2 \pm 0.21(9.0-9.5)$ \\
\hline Lip region height & 4.0 & $4.1 \pm 0.24(4.0-4.5)$ & $4.2 \pm 0.41(4.0-5.0)$ \\
\hline Amphidial aperture & 4.5 & $5.2 \pm 0.21(5.0-5.5)$ & $5.2 \pm 0.21(5.0-5.5)$ \\
\hline Odontostyle length & 8.5 & $8.4 \pm 0.36(8.0-9.0)$ & 8.5 \\
\hline Odontophore length & 9.0 & $9.2 \pm 0.24(9.0-9.5)$ & $8.8 \pm 0.24(8.5-9.0)$ \\
\hline Total stylet length & 17.5 & $17.4 \pm 0.49(17.0-18.5)$ & $17.2 \pm 0.24(17.0-17.5)$ \\
\hline Guiding ring from anterior end & 6.0 & $6.9 \pm 0.5(6.0-7.0)$ & $6.1 \pm 0.42(5.5-6.5)$ \\
\hline Nerve ring from anterior end & 74 & $66.2 \pm 4.2(61-73)$ & $66.9 \pm 4.6(62-73)$ \\
\hline Neck length & 196 & $183.0 \pm 8.6(170-195)$ & $185.4 \pm 7.5(178-197)$ \\
\hline Expanded part of pharynx & 82 & $75.8 \pm 2.9(73-80)$ & $75.6 \pm 4.4(68-80)$ \\
\hline Cardia length & 9.0 & $8.6 \pm 2.2(6.0-11)$ & 9.0 \\
\hline Anterior genital branch & 114 & $123.8 \pm 17.6(98-147)$ & - \\
\hline Posterior genital branch & 70 & $49.1 \pm 8.9(39-60)$ & - \\
\hline Vaginal length & 10.5 & $9.2 \pm 0.48(8.5-10)$ & - \\
\hline Vulva from anterior end & 519 & $468.6 \pm 54.0(421-571)$ & - \\
\hline Prerectum length & 58 & $47.4 \pm 5.7(39-55)$ & $79.1 \pm 8.1(68-91)$ \\
\hline Rectum length & 14 & $14.9 \pm 0.99(13-15)$ & $19.6 \pm 1.2(17-20)$ \\
\hline Tail length & 21 & $21.3 \pm 0.39(21-22)$ & $19.3 \pm 1.06(17-20)$ \\
\hline Spicules length & - & - & $20.8 \pm 1.60(19-23)$ \\
\hline Lateral guiding pieces & - & - & $5.7 \pm 0.21(5.0-6.0)$ \\
\hline Ventromedian supplements & - & - & 3 \\
\hline
\end{tabular}


having longer odontostyle (8.0-9.0 vs 6.5-7.5 $\mu \mathrm{m})$; pharyngeal expansion gradual (vs abrupt); slightly shorter pharyngeal expansion (73-82 vs 83-93 $\mu \mathrm{m}$ ); slightly posterior vulva position (V=65.7-68.5 vs 61-65); longer posterior genital branch with distinct sphincter and rudimentary oviduct (39-60 vs 8.0 $10 \mu \mathrm{m}$, sphincter and rudimentary oviduct absent) and fewer ventromedian supplements (3 vs 4-6).

The new species differs from $T$. arakii in having differently shaped (lips moderately separate and angular vs lips rounded and amalgamated) and wider lip region (9.0-10.0 vs 6.0-7.0 $\mu \mathrm{m})$; wider amphidial aperture (4.5-5.5 vs 3.0-4.0 $\mu \mathrm{m}$ ); absence of labial disc (vs present); higher b (3.4-4.3 vs 2.8-3.1) and c' (1.4-1.6 vs 1.0-1.1) ratios; shorter pharyngeal expansion (73-82 vs 88-96 $\mu \mathrm{m})$ and presence of posterior sac (vs absent).

In the presence of gradual pharyngeal expansion and long posterior sac, the new species comes close to T. stecki Steiner, 1914 and T. vulvulatus Rahman et al., 1987. However, it differs from T. stecki, in having wider amphidial aperture (4.5-5.5 vs 3.0-3.5 $\mu \mathrm{m})$; longer odontostyle $(8.0-9.0$ vs 5.0-5.5 $\mu \mathrm{m})$, odontophore with asymmetrical basal knobs (vs symmetrical); slightly shorter pharynx and its expansion (170-196 vs 203-236 $\mu \mathrm{m}, 73-82$ vs 89-102 $\mu \mathrm{m})$; lower c (28-37 vs 44-59) and higher c' (1.4-1.6 vs $0.7-0.9)$ ratios.

The new species differs from T. vulvulatus in having a longer odontostyle $(8.0-9.0$ vs $6.0-7.0 \mu \mathrm{m})$, odontophore with asymmetrical basal knobs (vs symmetrical); vulval lips symmetrical (vs asymmetrical); lower c (28-37 vs 40-61), higher c' (1.4-1.6 vs 0.7-1.0) ratios and smaller spicules (19-23 vs 28-32 $\mu \mathrm{m})$.

In the presence of gradual pharyngeal expansion and long posterior sac the new species also comes close to T. australis Yeates, 1979 but differs in having longer body ( $0.61-0.85 \mathrm{vs} 0.50-0.54 \mathrm{~mm})$; wider amphidial aperture (4.5-5.5 vs 2.0-2.5 $\mu \mathrm{m})$; longer odontostyle (8.0-9.0 vs 4.5-5.0 $\mu \mathrm{m})$ and odontophore (9.0-9.5 vs $5.0 \mu \mathrm{m})$; knobs of odontophore asymmetrical (vs symmetrical); longer posterior genital branch with distinct sphincter and rudimentary oviduct (39-70 vs $25 \mu \mathrm{m}$, sphincter and rudimentary oviduct absent) and presence of male (vs absent).

Tylencholaimus shamimi sp. nov.

urn:lsid:zoobank.org:act:8EA31D0A-727E-44BD-923C-364BB928F60E

Figs 9-10, Table 7

\section{Diagnosis}

Tylencholaimus shamimi sp. nov. is characterized by having $0.57-0.71 \mathrm{~mm}$ long body; lip region offset by constriction, lips slightly elevated; odontostyle 6.0-7.0 $\mu \mathrm{m}$, odontophore $8.5-9.5 \mu \mathrm{m}$ with basal thickening, total stylet length $14.5-16.0 \mu \mathrm{m}$; pharynx with slightly muscular anterior part, expanding gradually into the cylindrical basal bulb occupying about $38-44 \%$ of total neck length; female genital system amphidelphic; vulva a transverse slit; tail short, convex-conoid with bluntly rounded terminus and males with 19-23 $\mu \mathrm{m}$ long spicules, lateral guiding pieces and two to three spaced ventromedian supplements.

\section{Etymology}

The new species is named after Prof. Mohammad Shamim Jairajpuri in recognition of his contribution to nematode taxonomy.

\section{Material examined}

\section{Holotype}

INDIA • 1 क; Kerala State, Idukki, Kudyathoor; 949'37.2" N, 76047'45.6" E; 5-15 cm depth; 31 Oct. 2017; roots of grasses (unidentified); slide reference number AMU/ZD/NC/Tylencholaimus shamimi/1. 
Paratypes

INDIA -4 우, 2 § $\overbrace{}^{\lambda}$; same collection data as for holotype; slides reference number AMU/ZD/NC/ Tylencholaimus shamimi/2-4・1 , 1 \%; same collection data as for holotype; slides reference number AMU/ZD/NC/Tylencholaimus shamimi/5-6, nematode collection of the Zoological Survey of India, Kolkata, India.

\section{Other material}

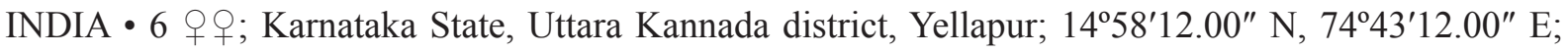
5-15 cm depth; 29 Oct. 2018; roots of grasses and shrubs (unidentified); slides reference number AMU/ $\mathrm{ZD} / \mathrm{NC} /$ Tylencholaimus shamimi/7-9.

\section{Type habitat and locality}

Soil sample collected from around the roots of grasses (unidentified) from Kudyathoor, Idukki district, Kerala State.

\section{Other habitat and locality}

Soil sample collected from around the roots of grasses and shrubs (unidentified) from Yellapur, Uttara Kannada district, Karnataka State.

\section{Description}

\section{Female}

Slender, small sized nematodes, slightly curved ventrad upon fixation; body cylindrical, tapering gradually towards both extremities. Cuticle with two distinct layers, $1.0-1.5 \mu \mathrm{m}$ thick at anterior region, 1.5-2.0 $\mu \mathrm{m}$ at midbody and $2.5-3.0 \mu \mathrm{m}$ on tail. Outer cuticle thin, finely striated; inner layer thick, loose, its outline irregular, with radial refractive elements. Lateral chords occupying about $23-32 \%$ of the midbody diameter. Dorsal, ventral and lateral body pores indistinct. Lip region narrow, cap-like, offset by constriction, 2.0-2.6 times as wide as high or about $1 / 3$ of the body diameter at neck base. Lips rounded, amalgamated, inner part slightly elevated. Labial and cephalic papillae distinct but not interfering with the labial contour. Amphids small, cup-shaped, their aperture occupying about $1 / 3$ of lip region diameter. Stoma a truncate cone. Odontostyle short, cylindrical, $0.8-0.9$ times the lip region diameter long, its aperture about $1 / 4$ to $1 / 3$ of the odontostyle length. Odontophore simple rod-like, with basal thickening, 1.3-1.5 times the odontostyle length. Guiding ring simple, refractive, at 0.6-0.9 times lip region diameter from anterior end. Pharynx consisting of a slightly muscular anterior part, expanding gradually into a cylindrical basal bulb, with thickwalled lumen, occupying about $38-44 \%$ of total neck length. Pharyngeal gland nuclei and their orifices are located as follows: $\mathrm{DO}=60-63, \mathrm{DN}=62-65, \mathrm{DO}-\mathrm{DN}=1.6-2.3, \mathrm{~S} 1 \mathrm{~N} 1=75-77, \mathrm{~S} 1 \mathrm{~N} 2=80-83, \mathrm{~S} 2 \mathrm{~N}=$ $89-90, \mathrm{~S} 2 \mathrm{O}=91-92$. Nerve ring located at $38-42 \%$ of neck length from anterior end. Cardia rounded to conoid, about $1 / 4$ to $2 / 5$ of the corresponding body diameter long.

Genital system didelphic-amphidelphic. Ovaries reflexed, measuring 43-89 $\mu \mathrm{m}$ (anterior) and 36-54 $\mu \mathrm{m}$ (posterior) long, not reaching the oviduct-uterus junction; oocytes arranged in single row except near tip. Oviduct joining the ovary subterminally, measuring $42-74 \mu \mathrm{m}$ (anterior) and 33-66 $\mu \mathrm{m}$ (posterior) long, consisting of a slender distal portion and a well developed pars dilatata. Oviduct-uterus junction marked with well-developed sphincter. Uterus short and tubular, measuring $28-41 \mu \mathrm{m}$ (anterior) and 26-45 $\mu \mathrm{m}$ (posterior). Sperm cell rarely present in the genital tract. Vagina cylindrical, extending inwards for $10-13 \mu \mathrm{m}$ or about $1 / 2(48-54 \%)$ of midbody diameter; pars proximalis vaginae $8.0-10 \times$ 4.0-6.0 $\mu \mathrm{m}$, encircled by circular muscles; pars distalis vaginae 3.0-3.5 $\mu \mathrm{m}$ with slightly curved walls; pars refringens absent. Vulva apparently a transverse slit. Prerectum 3.2-5.6 and rectum 0.8-1.4 anal body diameter long. Tail short, convex-conoid, $0.8-1.0$ times anal body diameter long, with a pair of caudal pores on each side and a distinct terminal pore. 

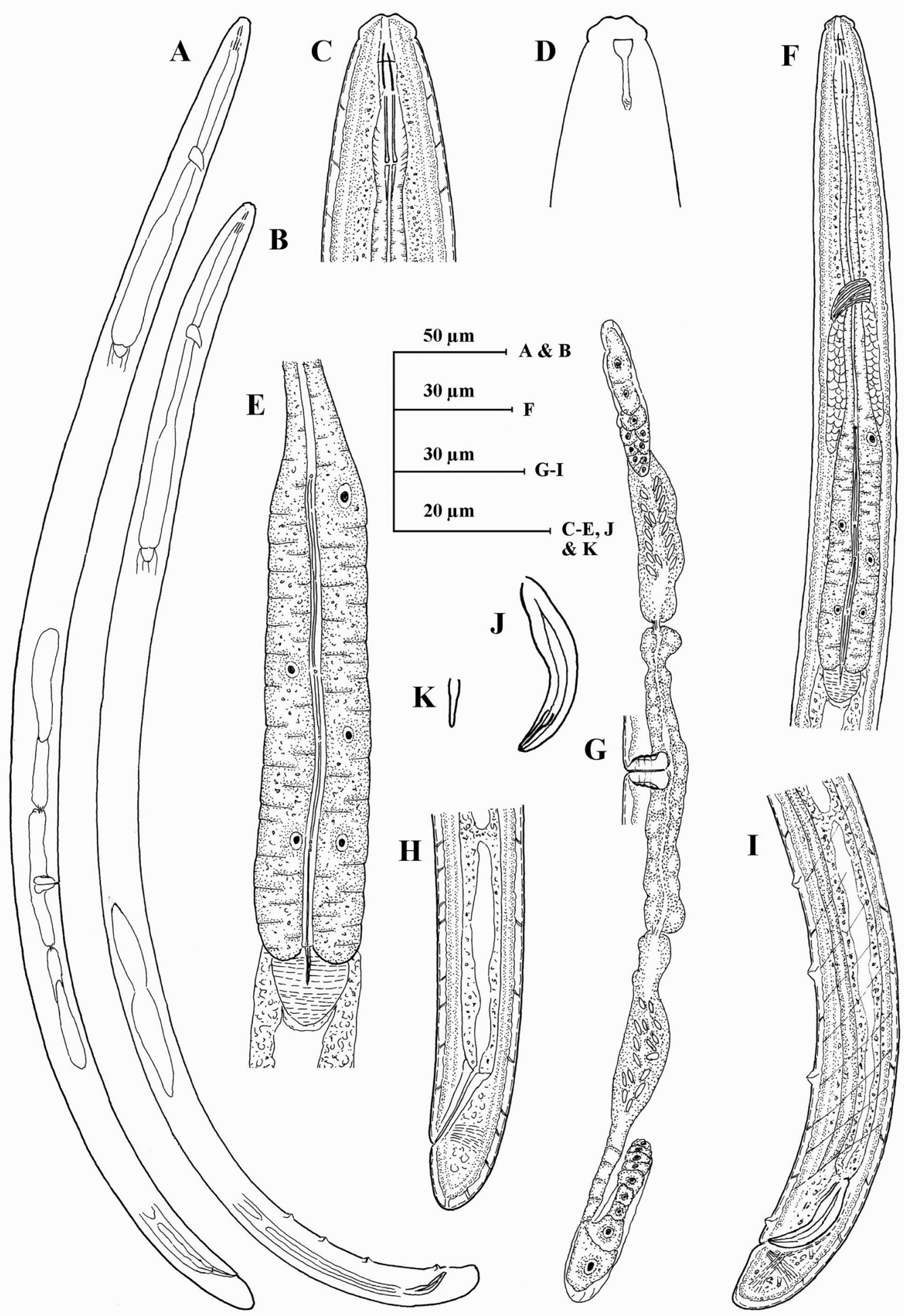

Fig. 9. Tylencholaimus shamimi sp. nov. A, G. Paratype 5, $q$ (slide 4). B, I-K. Paratype 6, ठ̊ (slide 5). C-F, H. Holotype, o (AMU/ZD/NC/Tylencholaimus shamimi/1). A. Entire female. B. Entire male. C. Female anterior region. D. Female anterior region showing amphid. E. Female expanded part of pharynx. F. Female pharyngeal region. G. Female genital system. H. Female posterior region. I. Male posterior region. J. Spicule. K. Lateral guiding piece. 

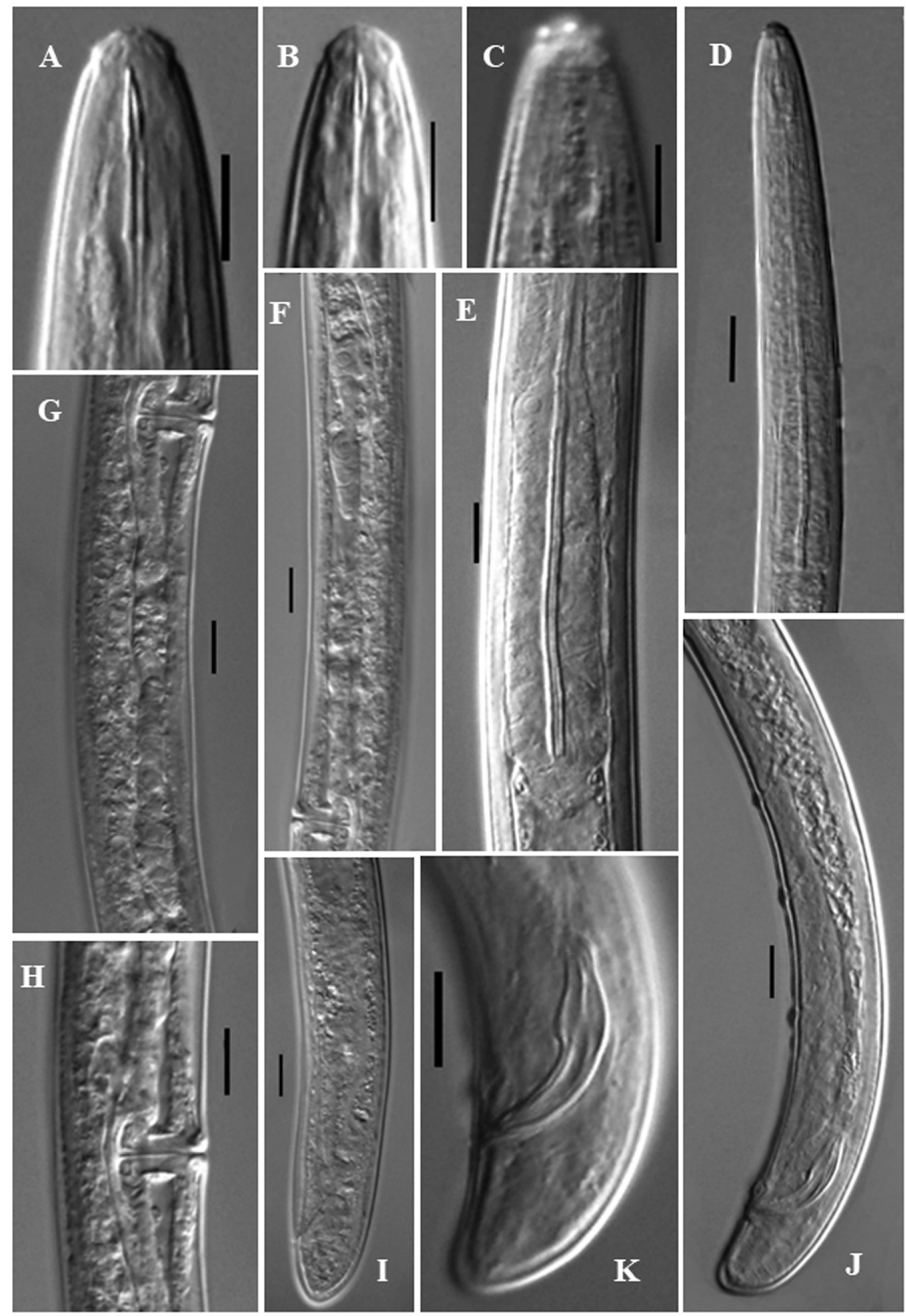

Fig. 10. Tylencholaimus shamimi sp. nov. (LM photographs). A, C, E, F-H. Holotype, $q$ (AMU/ZD/NC/ Tylencholaimus shamimi/1). B, D, I. Paratype 5, 9 (slide 4). J-K. Paratype 6, ô (slide 5). A-B. Female anterior region. C. Female anterior region showing amphid. D. Female pharyngeal region. E. Female expanded part of pharynx. F-G. Female genital system. H. Vulval region. I. Female posterior region. J. Male posterior region. K. Male posterior end. Scale bars: A-C, E-K $=10 \mu \mathrm{m} ; \mathrm{D}=20 \mu \mathrm{m}$. 


\section{Male}

General morphology similar to that of female, except for posterior region being more ventrally curved. Genital system diorchic, testes opposed, sperm cell spindle-shaped, 8.0-9.0 $\mu \mathrm{m}$ long. In addition to adcloacal pair, situated at 5.0-6.0 $\mu \mathrm{m}$ from cloacal aperture, there are two to three spaced ventromedian supplements, located outside the range of spicules; first one at 32-36 $\mu \mathrm{m}$ from adcloacal pair, second at $23-35 \mu \mathrm{m}$ from first and the third $(\mathrm{n}=1)$ at $20 \mu \mathrm{m}$ from second ventromedian supplement. Spicules typically dorylaimoid, curved ventrad, relatively slender, 4.6-4.8 times as long as wide and 1.4 times as long as cloacal body diameter, dorsal contour regularly convex, ventral contour bearing a moderately developed hump and hollow, curvature $128-131^{\circ}$, head occupying about $10-13 \%$ of total spicules length, median pieces $10.5-14.3$ times as long as wide, occupying about $30-40 \%$ of the spicules maximum width, reaching the spicules tip, posterior end 1.5-2.0 $\mu \mathrm{m}$ wide. Lateral guiding pieces distinct, rod-like, 4.3-4.6 times as long as wide or about $1 / 3$ of the spicules length. Prerectum 3.9-5.5 and rectum 1.5-1.6 cloacal body diameter long. Tail short, convex-conoid, with rounded terminus, 0.9-1.0 times cloacal body diameter long, with a pair of caudal pores on each side and a distinct terminal pore.

\section{Taxonomic remarks}

By its small body size; gradual pharyngeal expansion and amphidelphic female genital system, the new species comes close to T. innebus Ahmad \& Jairajpuri, 1979; T. congestus Loof \& Jairajpuri, 1968; T. mongolicus Andrássy, 1967 and T. suryawanshii Ali \& Chisty, 1972, but differs from the former in having a cap-like lip region with rounded, amalgamated lips (vs lip region expanded, broad and labial papillae elevated); smaller amphidial aperture ( $1 / 3 \mathrm{vs} 2 / 3$ of the lip region diameter); odontostyle with narrow lumen (vs wide lumen); shorter expanded part of pharynx (38-44 vs $36-37 \%$ of the total neck length); differently shaped cardia (rounded to conoid vs hemispheroid); longer prerectum (53-86 vs $26-50 \mu \mathrm{m}$ ); differently shaped tail (convex-conoid vs hemispheroid) and presence of male (vs absent).

The new species differs from $T$. congestus in having a shorter body size $(0.57-0.71$ vs $0.72-0.83 \mathrm{~mm})$; smaller amphidial aperture ( $1 / 3 \mathrm{vs} 1 / 2$ of the lip region diameter); shorter pharynx and its expansion (166172 vs $230 \mu \mathrm{m}, 64-75$ vs $80 \mu \mathrm{m}$ ) and presence of male (vs absent).

The new species differs from T. mongolicus in having a longer body size $(0.57-0.71 \mathrm{vs} 0.52-0.57 \mathrm{~mm})$; narrower lip region (7.0-8.0 vs $11-12 \mu \mathrm{m})$; higher c (36-63 vs $21-30)$ and slightly lower c' (0.8-1.0 vs $1.0-1.2)$ ratios; comparatively posterior vulva position $(\mathrm{V}=58-63$ vs $52-55)$ and presence of male (vs absent).

The new species differs from $T$. suryawanshii in having a narrower lip region $(7.0-8.0 \mathrm{vs} 9.0 \mu \mathrm{m})$; smaller amphidial aperture ( $1 / 3$ vs $2 / 3$ of the lip region diameter); shorter odontostyle $(6.0-7.0$ vs $8.5 \mu \mathrm{m})$ and odontophore (8.5-9.5 vs $12 \mu \mathrm{m})$; longer prerectum (53-86 vs $30 \mu \mathrm{m})$ and presence of male (vs absent).

In the presence of gradual pharyngeal expansion, the new species also comes close to $T$. sinensis Li et al., 2008 and T. teres Thorne, 1939 but differs from the former in having a smaller body size (0.570.71 vs $0.76-0.93 \mathrm{~mm})$; smaller amphidial aperture (2.0-3.0 vs 4.0-5.0 $\mu \mathrm{m})$; comparatively posterior vulva position ( $\mathrm{V}=58-63$ vs $57.0-57.5)$; longer pharyngeal expansion (38-44 vs 35-36\% of total neck length); shorter prerectum (53-85 vs 100-105 $\mu \mathrm{m})$ and shorter spicules (23-24 vs $32 \mu \mathrm{m})$.

The new species differs from $T$. teres in having a smaller body size $(0.57-0.71 \mathrm{vs} 0.75-1.2 \mathrm{~mm})$, slightly smaller amphidial aperture (2.0-3.0 vs 3.0-4.0 $\mu \mathrm{m})$; shorter pharynx and its expansion (166-172 vs 184-247 $\mu \mathrm{m}, 65-74$ vs $76-115 \mu \mathrm{m})$; shorter prerectum (53-85 vs $105-202 \mu \mathrm{m}, 3.2-5.6$ vs $6.8-9.5$ times corresponding body diameter); shorter (23-24 vs $28-37 \mu \mathrm{m})$ and differently shaped spicules (robust vs slender, $4.6-4.8$ vs 6.2 times as long as wide). 
Table 7. Measurements of Tylencholaimus shamimi sp. nov. All measurements are in $\mu \mathrm{m}$ and in the form: mean \pm s.d. (range).

\begin{tabular}{|c|c|c|c|c|}
\hline Localities & & Type populat & tion & Yellapur population \\
\hline Characters & $\begin{array}{c}\text { Holotype } \\
\text { female }\end{array}$ & Paratypes females & Paratypes males & Females \\
\hline $\bar{n}$ & & 5 & 3 & 6 \\
\hline $\mathrm{L}$ & 709 & $629.9 \pm 53.1(573-715)$ & $625.8 \pm 38.3(583-676)$ & $617.8 \pm 39.9(575-680)$ \\
\hline $\mathrm{a}$ & 28.9 & $26.9 \pm 1.4(24.3-28.3)$ & $30.4 \pm 1.7(28.6-32.8)$ & $26.7 \pm 1.6(23.9-29.0)$ \\
\hline $\mathrm{b}$ & 4.2 & $3.7 \pm 0.35(3.3-4.2)$ & $3.6 \pm 0.21(3.5-4.0)$ & $3.6 \pm 0.23(3.3-3.9)$ \\
\hline $\mathrm{c}$ & 48.2 & $42.9 \pm 3.9(36.5-63.7)$ & $39.1 \pm 1.4(37.1-40.5)$ & $39.8 \pm 1.7(37.7-43.3)$ \\
\hline c' & 0.88 & $0.90 \pm 0.06(0.82-1.0)$ & $0.97 \pm 0.02(0.93-1.0)$ & $0.96 \pm 0.06(0.84-1.0)$ \\
\hline $\mathrm{V}$ & 60.7 & $63.0 \pm 0.69(61.9-63.7)$ & - & $61.0 \pm 1.3(58.2-62.7)$ \\
\hline G1 & 19.6 & $19.4 \pm 0.81(18.4-20.6)$ & - & $17.9 \pm 0.74(17.3-18.7)$ \\
\hline G2 & 19.3 & $15.6 \pm 2.5(11.7-18.9)$ & - & $15.0 \pm 1.9(12.2-17.8)$ \\
\hline Body diameter at neck base & 24 & $22.9 \pm 0.99(21.5-24.5)$ & $20.6 \pm 0.80(19.5-21.5)$ & $21.3 \pm 1.4(20-25)$ \\
\hline Body diameter at mid body & 25 & $23.3 \pm 1.3(21.5-25.0)$ & $20.5 \pm 0.80(19.5-21.0)$ & $23.1 \pm 2.6(20-28)$ \\
\hline Body diameter at anus & 17 & $16.2 \pm 0.78(15.5-17.5)$ & $16.3 \pm 0.46(16.0-16.5)$ & $16.1 \pm 1.2(14-19)$ \\
\hline Lip region diameter & 7.0 & $7.1 \pm 0.39(7.0-8.0)$ & $7.1 \pm 0.23(7.0-7.5)$ & $7.5 \pm 0.36(7.0-8.0)$ \\
\hline Lip region height & 3.5 & $3.2 \pm 0.4(3.0-4.0)$ & 3.5 & $3.1 \pm 0.18(3.0-3.5)$ \\
\hline Amphidial aperture & 2.5 & $2.3 \pm 0.36(2.0-3.0)$ & $2.7 \pm 0.23(2.0-3.0)$ & $2.8 \pm 0.18(2.5-3.0)$ \\
\hline Odontostyle length & 6.5 & $6.3 \pm 0.30(6.0-7.0)$ & $6.2 \pm 0.46(6.0-6.5)$ & $6.2 \pm 0.18(6.0-6.5)$ \\
\hline Odontophore length & 9.5 & $8.8 \pm 0.30(8.5-9.5)$ & 8.5 & $8.6 \pm 0.23(8.5-9.0)$ \\
\hline Total stylet length & 16 & $15.5 \pm 0.64(14.5-16)$ & $14.8 \pm 0.4(14.5-15.0)$ & $15.1 \pm 0.40(15.0-15.5)$ \\
\hline Guiding ring from anterior end & 6.0 & $5.5 \pm 0.59(5.0-6.5)$ & $5.3 \pm 0.40(5.0-5.5)$ & $5.3 \pm 0.28(5.0-6.0)$ \\
\hline Nerve ring from anterior end & 71 & $69.3 \pm 2.2(66-72)$ & $69.9 \pm 3.33(66-74)$ & $66.4 \pm 1.5(64-69)$ \\
\hline Neck length & 168 & $169.3 \pm 2.4(166-172)$ & $172.8 \pm 6.0(165-180)$ & $169.3 \pm 1.6(166-170)$ \\
\hline Expanded part of pharynx & 65 & $72.1 \pm 1.7(69-73)$ & $71.2 \pm 4.68(64-75)$ & $70.3 \pm 2.2(67-74)$ \\
\hline Cardia length & 8.0 & $6.1 \pm 0.39(6.0-7.0)$ & $6.2 \pm 0.23(6.0-6.5)$ & $7.0 \pm 1.0(6.0-9.0)$ \\
\hline Anterior genital branch & 139 & $122.5 \pm 13.2(105-142)$ & - & $113.5 \pm 10.9(100-130)$ \\
\hline Posterior genital branch & 140 & $99.3 \pm 9.3(84-108)$ & - & $94.2 \pm 8.5(80-104)$ \\
\hline Vaginal length & 12 & $12.1 \pm 0.48(11-13)$ & - & $10.7 \pm 0.56(10-12)$ \\
\hline Vulva from anterior end & 431 & $396.7 \pm 31.1(358-442)$ & - & $376.8 \pm 21.8(353-418)$ \\
\hline Prerectum length & 62 & $62.9 \pm 10.7(53-83)$ & $75.7 \pm 8.40(65-86)$ & $77.4 \pm 7.4(62-85)$ \\
\hline Rectum length & 21 & $18.0 \pm 2.3(15-21)$ & $25.1 \pm 0.46(25.0-25.5)$ & $15.1 \pm 1.2(12-17)$ \\
\hline Tail length & 15 & $14.7 \pm 0.61(14-16)$ & $16.3 \pm 0.92(16-17)$ & $15.5 \pm 0.67(14-17)$ \\
\hline Spicules length & - & - & $23.3 \pm 0.46(23-24)$ & - \\
\hline Lateral guiding pieces & - & - & $6.6 \pm 0.23(6.0-7.0)$ & - \\
\hline Ventromedian supplements & - & - & $2-3$ & - \\
\hline
\end{tabular}


Tylencholaimus southindicus sp. nov. urn:1sid:zoobank.org:act:995DAB2D-59D1-4B2D-BD2E-07B06E46C5A5

Figs 11-12, Table 8

\section{Diagnosis}

Tylencholaimus southindicus sp. nov. is characterized by having $0.44-0.55 \mathrm{~mm}$ long, slender body; lip region offset by constriction, labial disc distinct; odontostyle 4.5-5.5 $\mu \mathrm{m}$, odontophore 5.0-6.0 $\mu \mathrm{m}$, total stylet length $10-11.5 \mu \mathrm{m}$; pharynx with slender anterior part expanding abruptly into a cylindrical basal bulb occupying about $39-43 \%$ of total neck length; female genital system monodelphic-prodelphic; posterior genital branch reduced to a simple sac, $22-54 \mu \mathrm{m}$ or $1.2-3.0$ times the midbody diameter long; vulva transverse; tail short, rounded to rounded-conoid; males with 17-18 $\mu \mathrm{m}$ long spicules, $4.5-5.5 \mu \mathrm{m}$ long lateral guiding pieces and two spaced ventromedian supplements.

\section{Etymology}

The new species is named Tylencholaimus southindicus sp. nov. because of its distribution in south India.

\section{Material examined}

\section{Holotype}

INDIA • 1 \%; Kerala State, Ernakulam district, Manikandanchal; $10^{\circ} 09^{\prime 2} 28.8^{\prime \prime}$ N, 76047'56.4" E; 5-15 cm depth; 28 Oct. 2017; roots of shrubs (unidentified); slide reference number AMU/ZD/NC/Tylencholaimus southindicus/1.

Paratypes

INDIA 9 우, 7 ふぇ; same collection data as for holotype; slides reference number AMU/ZD/NC/ Tylencholaimus southindicus/2-5 -1 क, 1 त; same collection data as for holotype; slide reference number AMU/ZD/NC/Tylencholaimus southindicus/6, nematode collection of the Zoological Survey of India, Kolkata, India.

\section{Other material}

INDIA • 16 우; Karnataka State, Kodagu district, Bhagamandala; $12^{\circ} 23^{\prime} 29.1^{\prime \prime}$ N, $75^{\circ} 31^{\prime} 50.0^{\prime \prime}$ E; 5-15 cm depth; 8 Nov. 2016; roots of shrubs and forest trees (unidentified); slides reference number AMU/ZD/NC/Tylencholaimus southindicus/7-13.

\section{Type habitat and locality}

Soil samples collected from around the roots of shrubs (unidentified) from Manikandanchal, Ernakulam district, Kerala State.

\section{Other habitat and locality}

Soil samples collected from around the roots of shrubs (unidentified) and forest trees (unidentified) from Bhagamandala, Kodagu district, Karnataka State.

\section{Description}

\section{Female}

Small sized nematodes, $0.44-0.55 \mathrm{~mm}$ long; curved ventrad upon fixation; body cylindrical, tapering gradually towards both extremities but more so towards the anterior end. Cuticle with two distinct layers, $1.0-1.5 \mu \mathrm{m}$ thick at midbody and $1.5-2 \mu \mathrm{m}$ on tail. Outer cuticle finely striated; inner layer thick, with radial refractive elements. Lateral chords occupying about $23-31 \%$ of midbody diameter. Lateral, dorsal and ventral body pores indistinct. Lip region cap-like, offset by distinct constriction, 1.4-2.0 
times as wide as high or about $1 / 3$ of the body diameter at neck base. Lips rounded, amalgamated, inner part elevated, transformed into a labial disc-like structure. Amphids small, cup-shaped, their aperture occupying about $1 / 3$ to $2 / 5$ of lip region diameter. Stoma a truncate cone. Odontostyle short, slender, anterior end slightly thick, $0.8-1.0$ times the lip region diameter long, its aperture about $1 / 5$ to $1 / 3$ of the odontostyle length. Odontophore rod-like, with minute basal knobs, 1.0-1.2 times the odontostyle length. Guiding ring simple, refractive, at 0.6-0.8 times lip region diameter from anterior end. Pharynx consisting of a slender, slightly muscular anterior part, expanding abruptly into a cylindrical basal bulb, with thick-walled lumen, occupying about $39-43 \%$ of total neck length. Pharyngeal gland nuclei and their orifices are located as follows: $\mathrm{DO}=62-65, \mathrm{DN}=65-68, \mathrm{DN}-\mathrm{DO}=1.9-3.4, \mathrm{~S} 1 \mathrm{~N} 1=75-79$, $\mathrm{S} 1 \mathrm{~N} 2=79-82, \mathrm{~S} 2 \mathrm{~N}=88-90, \mathrm{~S} 2 \mathrm{O}=90-92$. Nerve ring located at $36-43 \%$ of neck length from anterior region. Cardia rounded to conoid, about $1 / 3$ of the corresponding body diameter long.

Genital system monodelphic-prodelphic. Ovary reflexed, measuring 32-86 $\mu \mathrm{m}$ long; oocytes arranged in single row except near tip. Oviduct joining the ovary subterminally, measuring 53-102 $\mu \mathrm{m}$, with a welldeveloped pars dilatata. Oviduct-uterus junction marked by well-developed sphincter. Uterus slightly long and tubular, measuring $27-53 \mu \mathrm{m}$. Posterior genital branch reduced to simple sac, measuring $22-54 \mu \mathrm{m}$ or $1.2-3.0$ times midbody diameter. Sperm cell present throughout the genital tract. Vagina cylindrical, extending inwards, $8.0-10 \mu \mathrm{m}$ or about $1 / 2$ to $3 / 5(46-61 \%)$ of midbody diameter; pars proximalis vaginae $5.0-7.0 \times 3.0-5.0 \mu \mathrm{m}$, encircled by circular muscles; pars distalis vaginae short, 2.0-3.0 $\mu \mathrm{m}$ with slightly curved walls; pars refringens absent. Vulva a transverse slit. Prerectum 3.4-6.6 and rectum 0.9-1.2 times anal body diameter long. Tail short, rounded to rounded-conoid, 0.6-0.9 times anal body diameter long, with a pair of subdorsal and a prominent terminal caudal pore present.

\section{Male}

General morphology similar to that of female, except for posterior region being more ventrally curved. Genital system diorchic, testes opposed, sperm cell spindle-shaped. In addition to adcloacal pair at $4.0-5.0 \mu \mathrm{m}$ from cloacal aperture, there are two ventromedian supplements, located outside the range of spicules, first one at $22-31 \mu \mathrm{m}$ from adcloacal pair, second at 16-31 $\mu \mathrm{m}$ from first ventromedian supplement. Spicules typically dorylaimoid, curved ventrad, 4.3-5.1 times as long as wide and 1.3-1.5 times as long as body diameter at level of cloacal aperture, dorsal contour regularly convex, ventral contour bearing a moderately developed hump and hollow, curvature $125-130^{\circ}$, head occupying about $10-11 \%$ of total spicules length, median pieces $7.5-11.3$ times as long as wide, occupying about $37-$ $57 \%$ of the spicules maximum width, reaching the spicules tip, posterior end $1.5-2.0 \mu \mathrm{m}$ wide. Lateral guiding pieces distinct, rod-like, about 3.3-5.0 times as long as wide or about $1 / 4$ to $1 / 3$ of the spicules length. Prerectum 5.1-7.9 and rectum 1.5-1.7 times cloacal body diameter long. Tail short, rounded to conoid, $0.7-1.0$ times cloacal body diameter long; a pair of subdorsal and a prominent terminal caudal pore present.

\section{Taxonomic remarks}

In the presence of labial disc, abrupt pharyngeal expansion, presence of posterior uterine sac and short rounded to hemispheroid tail, the new species comes close to T. imperamus Mohilal \& Dhanachand, 2003; T. discus Golhasan et al., 2019 and T. constrictus Vinciguerra, 1986 but differs from the former in the shape and size of labial disc (large vs small); distinctly offset lip region (vs lip region almost continuous); longer post-uterine sac (22-54 vs 9.0-18 $\mu \mathrm{m})$; smaller spicules $(17-18$ vs $24-25 \mu \mathrm{m})$ and fewer ventromedian supplements (2 vs 3 ).

The new species differs from $T$. discus in having a shorter body size $(0.44-0.55$ vs $0.65-0.76 \mathrm{~mm})$; comparatively smaller amphidial aperture $(2.0-2.5$ vs 3.0-4.0 $\mu \mathrm{m})$; shorter total stylet length $(10.0-$ 11.5 vs $15.0-17.5 \mu \mathrm{m}$, odontostyle $4.5-5.5$ vs $6.0-7.0 \mu \mathrm{m}$, odontophore 5.0-6.0 vs $9.0-10.5 \mu \mathrm{m}$ ); odontophore with minute basal knobs (vs large knobs); shorter pharynx and its expansion (142-164 vs 

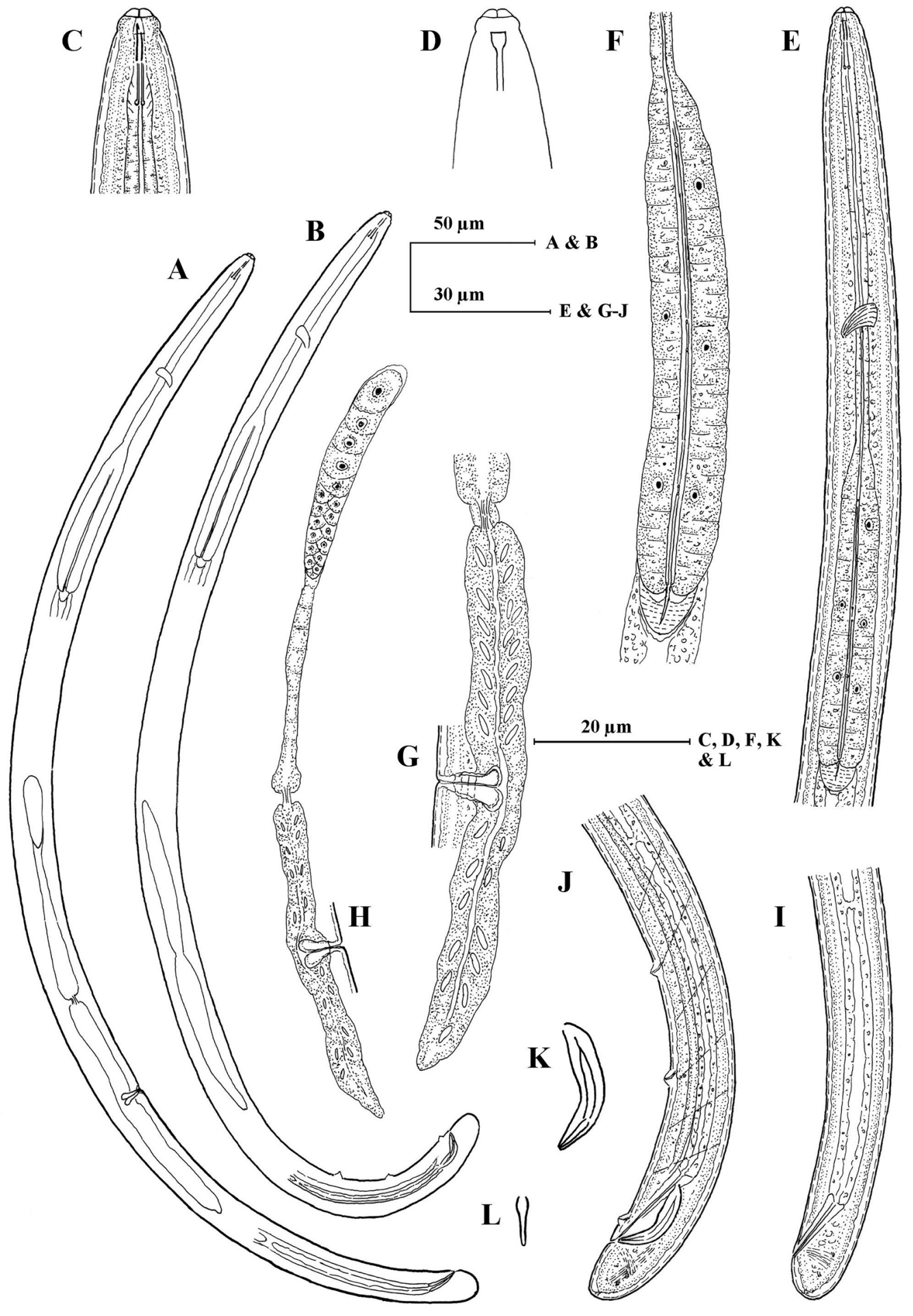

Fig. 11. Tylencholaimus southindicus sp. nov. A. Paratype 1 , $q$ (slide 2). B, J-L. Paratype 11, § (slide 5). C, E. Paratype 5, $q$ (slide 3). D, I. Holotype,, (AMU/ZD/NC/Tylencholaimus southindicus/1). F. Paratype 3, $q$ (slide 2). G. Paratype 4, $q$ (slide 2). H. Paratype 13, $q$ (slide 5). A. Entire female. B. Entire male. C. Female anterior region. D. Female anterior region showing amphid. E. Female pharyngeal region. F. Female expanded part of pharynx. G-H. Female genital system. I. Female posterior region. J. Male posterior region. K. Spicule. L. Lateral guiding piece. 


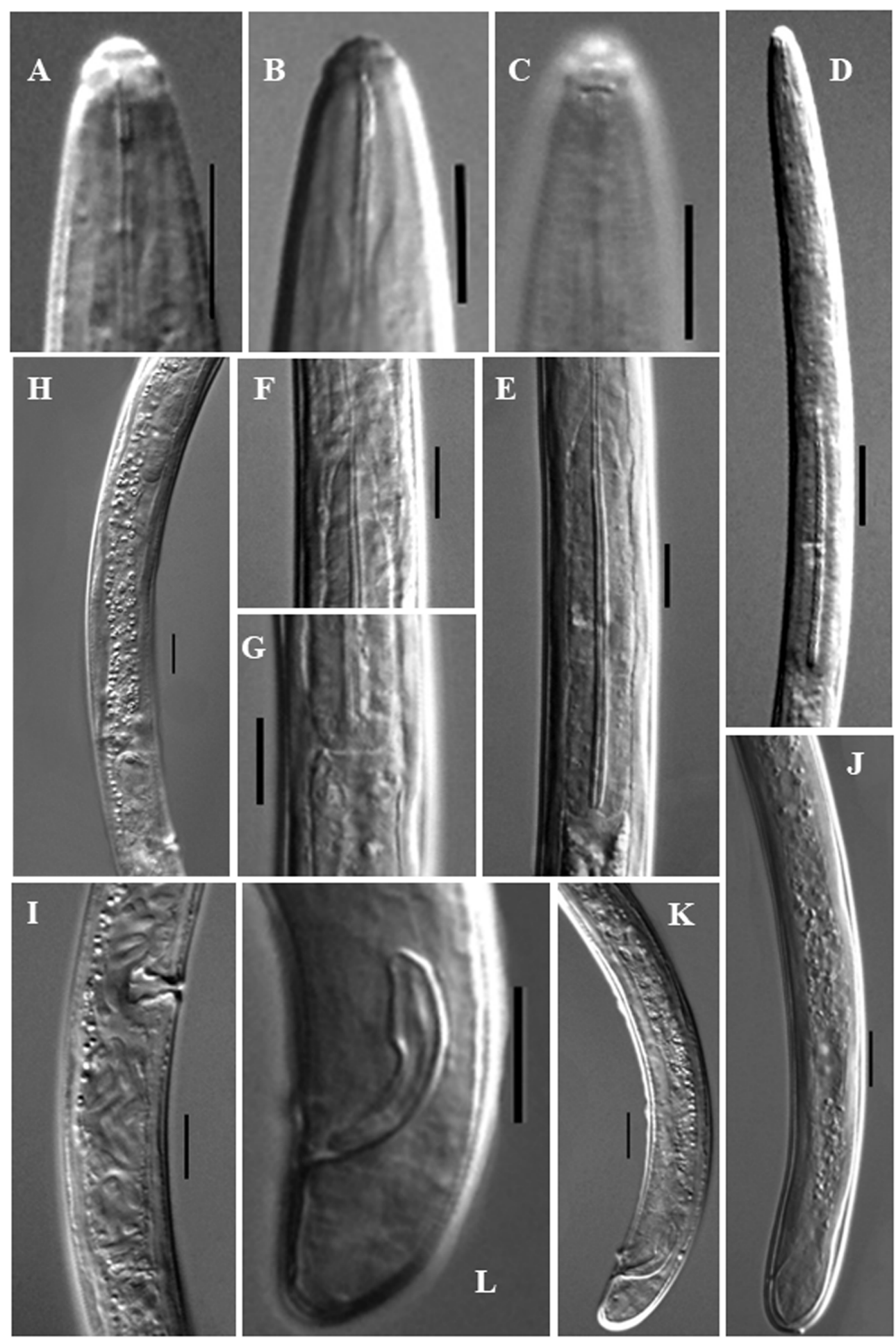

Fig. 12. Tylencholaimus southindicus sp. nov. (LM photographs). A. Paratype 1, $q$ (slide 2). B, E, G. Holotype, $q$ (AMU/ZD/NC/Tylencholaimus southindicus/1). C-D, F. Paratype 5, $q$ (slide 3). H-J. Paratype 4, $q$ (slide 2). K-L. Paratype 3, ô (slide 2). A-B. Female anterior region. C. Female anterior region showing amphid. D. Female pharyngeal region. E. Female expanded part of pharynx. F. Female pharyngeal expansion. G. Female pharyngo-intestinal junction. H-I. Female genital system. J. Female posterior region. K. Male posterior region. L. Male posterior end showing spicules. Scale bars: $\mathrm{A}-\mathrm{C}, \mathrm{E}-\mathrm{L}=10 \mu \mathrm{m} ; \mathrm{D}=20 \mu \mathrm{m}$. 
Table 8. Measurements of Tylencholaimus southindicus sp. nov. All measurements are in $\mu \mathrm{m}$ and in the form: mean \pm s.d. (range).

\begin{tabular}{|c|c|c|c|c|}
\hline \multirow{2}{*}{$\begin{array}{l}\text { Localities } \\
\text { Characters }\end{array}$} & \multicolumn{3}{|c|}{ Type population } & \multirow{2}{*}{$\begin{array}{c}\begin{array}{c}\text { Bhagamandala } \\
\text { population }\end{array} \\
\text { Females }\end{array}$} \\
\hline & $\begin{array}{c}\text { Holotype } \\
\text { female }\end{array}$ & Paratypes females & Paratypes males & \\
\hline $\mathrm{n}$ & & 10 & 8 & 16 \\
\hline $\mathrm{L}$ & 508 & $493.8 \pm 18.5(468-527)$ & $488.1 \pm 21.2(452-531)$ & $504.2 \pm 28.2(441-558)$ \\
\hline a & 30.5 & $28.6 \pm 1.2(26.6-30.7)$ & $29.3 \pm 0.51(29.2-30.8)$ & $29.9 \pm 2.13(25.9-33.9)$ \\
\hline $\mathrm{b}$ & 3.1 & $3.2 \pm 0.1(2.9-3.4)$ & $3.1 \pm 0.07(3.0-3.2)$ & $3.2 \pm 0.14(3.0-3.5)$ \\
\hline $\mathrm{c}$ & 47.1 & $50.5 \pm 2.2(47.1-53.8)$ & $44.0 \pm 3.10(40.5-49.8)$ & $45.6 \pm 5.1(39.1-62.0)$ \\
\hline$c^{\prime}$ & 0.78 & $0.72 \pm 0.4(0.69-0.83)$ & $0.88 \pm 0.05(0.83-1.0)$ & $0.86 \pm 0.07(0.67-1.0)$ \\
\hline $\mathrm{V}$ & 70.5 & $68.7 \pm 0.79(67.8-70.5)$ & - & $66.9 \pm 1.5(64.3-69.6)$ \\
\hline G1 & 26.4 & $26.4 \pm 1.9(22-29)$ & - & $25.7 \pm 1.8(22.9-29.9)$ \\
\hline G2 & 8.3 & $8.6 \pm 1.3(5.5-10.5)$ & - & $5.5 \pm 0.75(4.3-6.9)$ \\
\hline Body diameter at neck base & 17.6 & $17.2 \pm 0.75(15-18)$ & $16.5 \pm 0.58(15.5-17.5)$ & $16.9 \pm 0.57(15-17.5)$ \\
\hline Body diameter at mid body & 16.6 & $16.9 \pm 0.43(16-17.5)$ & $16.2 \pm 0.83(15.0-17.5)$ & $16.5 \pm 0.76(16-17)$ \\
\hline Body diameter at anus & 14 & $13.3 \pm 0.62(11-13.5)$ & $12.1 \pm 0.47(11.5-12.5)$ & $12.4 \pm 0.45(11-12)$ \\
\hline Lip region diameter & 5.5 & $5.4 \pm 0.35(5.0-6.0)$ & $5.5 \pm 0.33(5.0-6.0)$ & $5.4 \pm 0.43(5.0-6.0)$ \\
\hline Lip region height & 3.0 & $3.2 \pm 0.24(3.0-3.5)$ & $3.1 \pm 0.21(3.0-3.5)$ & $3.2 \pm 0.23(3.0-3.5)$ \\
\hline Amphidial aperture & 2.5 & $2.1 \pm 0.14(2.0-2.5)$ & $2.3 \pm 0.32(2.0-2.5)$ & $2.2 \pm 0.24(2.0-2.5)$ \\
\hline Odontostyle length & 5.0 & $4.9 \pm 0.20(4.5-5.5)$ & $5.2 \pm 0.23(5.0-5.5)$ & $4.8 \pm 0.11(4.5-5.5)$ \\
\hline Odontophore length & 6.0 & $5.6 \pm 0.3(5.0-6.0)$ & $5.6 \pm 0.24(5.5-6.0)$ & $5.5 \pm 0.27(5.0-6.0)$ \\
\hline Total stylet length & 11 & $10.6 \pm 0.36(10.0-11.5)$ & $10.7 \pm 0.38(10.5-11.5)$ & $10.4 \pm 0.27(10-11)$ \\
\hline Guiding ring from anterior end & 4.0 & $4.2 \pm 0.31(4.0-5.0)$ & $3.7 \pm 0.21(3.5-4.0)$ & $4.1 \pm 0.44(3.5-5.0)$ \\
\hline Nerve ring from anterior end & 66 & $62.8 \pm 2.1(59-66)$ & $61.4 \pm 1.2(59-62)$ & $62.8 \pm 1.8(58-66)$ \\
\hline Neck length & 159 & $155.7 \pm 4.7(149-164)$ & $156.1 \pm 4.6(152-162)$ & $151.6 \pm 5.7(142-164)$ \\
\hline Expanded part of pharynx & 69 & $65.9 \pm 2.6(61-71)$ & $68.6 \pm 2.1(66-71)$ & $62.9 \pm 1.9(60-67)$ \\
\hline Cardia length & 5.0 & $5.3 \pm 0.46(5.0-6.0)$ & $5.5 \pm 0.46(5.0-6.0)$ & $4.7 \pm 0.90(4.0-6.0)$ \\
\hline Anterior genital branch & 137 & $133.1 \pm 10.7(109-151)$ & - & $132.4 \pm 9.6(113-151)$ \\
\hline Posterior genital branch & 43 & $43.5 \pm 7.4(26-54)$ & - & $28.4 \pm 4.4(22-38)$ \\
\hline Vaginal length & 9.0 & $8.4 \pm 0.42(8.0-9.0)$ & - & $9.1 \pm 0.52(8.5-10)$ \\
\hline Vulva from anterior end & 358 & $339.3 \pm 13.6(320-364)$ & - & $344.8 \pm 23.8(292-377)$ \\
\hline Prerectum length & 69 & $65.2 \pm 9.02(50-80)$ & $78.4 \pm 11.6(61-102)$ & $65.4 \pm 8.2(54-86)$ \\
\hline Rectum length & 15 & $14.8 \pm 2.1(11.5-16.5)$ & $20.3 \pm 0.64(19-21)$ & $14.3 \pm 0.75(12.5-15.5)$ \\
\hline Tail length & 11 & $9.8 \pm 0.41(9.0-11)$ & $10.9 \pm 0.58(10.0-11.5)$ & $10.9 \pm 0.76(9.0-11)$ \\
\hline Spicules length & - & - & $17.2 \pm 0.47(17-18)$ & - \\
\hline Lateral guiding pieces & - & - & $4.9 \pm 0.24(4.5-5.5)$ & - \\
\hline Ventromedian supplements & - & - & 2 & - \\
\hline
\end{tabular}


230-259 $\mu \mathrm{m}, 56-71 \mathrm{vs} 98-125 \mu \mathrm{m})$; slightly shorter tail length (9.0-11 vs $13-17 \mu \mathrm{m})$ and presence of male (vs absent).

The new species differs from $T$. constrictus in having a shorter body size $(0.44-0.55$ vs $0.76-0.96 \mathrm{~mm})$; narrower lip region $(5.0-6.0$ vs 7.0-8.0 $\mu \mathrm{m})$; smaller amphidial aperture $(2.0-2.5$ vs $4 \mu \mathrm{m})$, shorter odontophore $(5.0-6.0$ vs $9.0-10 \mu \mathrm{m})$; longer post-uterine sac (22-54 vs 5.0-16 $\mu \mathrm{m})$; shorter spicules $(17-18$ vs $28 \mu \mathrm{m})$ and fewer ventromedian supplements (2 vs 5-6).

In the presence of long posterior uterine sac, the new species also comes close to T. longicaudatus PeñaSantiago \& Coomans, 1994 and T. conicaudatus Peña-Santiago \& Coomans, 1994 but differs from the former in having a shorter body size $(0.44-0.55$ vs $0.60-0.77 \mathrm{~mm})$; comparatively posterior vulva position $(\mathrm{V}=64-70$ vs $59-63)$; shorter post-uterine sac $(22-54 \mathrm{vs} 61-90 \mu \mathrm{m})$ and shorter tail $(9.0-11 \mathrm{vs}$ $31-41 \mu \mathrm{m}, \mathrm{c}=39-64$ vs $18-21, \mathrm{c}^{\prime}=0.6-1.0$ vs $\left.2.1-3.2\right)$.

The new species differs from $T$. conicaudatus in having a slightly narrow lip region (5.0-6.0 vs 7.0$8.0 \mu \mathrm{m}$ ); pharyngeal expansion abrupt (vs gradual); slightly posterior vulva position ( $\mathrm{V}=64-70 \mathrm{vs}$ 61-65); higher c (39-62 vs 21-24) and lower c' (0.6-1.0 vs 1.3-1.6) ratios; tail rounded (vs conoid) and presence of male (vs absent).

\section{Tylencholaimus striatus sp. nov. urn:1sid:zoobank.org:act:AB28078B-AE98-4000-844C-B8823170F355} Figs 13-14, Table 9

\section{Diagnosis}

Tylencholaimus striatus sp. nov. is characterized by having small sized, robust body, $0.30-0.34 \mathrm{~mm}$; distinctly striated cuticle; lip region set off by slight constriction; odontostyle 5.0-5.5 $\mu \mathrm{m}$; odontophore about as long as odontostyle with minute basal knobs, total stylet length 10-11 $\mu \mathrm{m}$; pharynx with slender anterior part, expanding abruptly into a cylindrical basal bulb occupying about $36-41 \%$ of total neck length; female genital system monodelphic-prodelphic; posterior genital branch reduced to a small uterine sac, 0.4-0.6 times the midbody diameter; vulva a transverse slit and tail short, cylindroid to conoid with bluntly rounded terminus.

\section{Etymology}

The new species is named Tylencholaimus striatus sp. nov. because of its distinctly striated cuticle.

\section{Material examined}

\section{Holotype}

INDIA • 1 क; Karnataka State, Kodagu district, Bhagamandala; $12^{\circ} 23^{\prime} 29.1^{\prime \prime}$ N, 75³1'50.0" E; 5-15 cm depth; 15 Nov. 2016; roots of shrubs and forest trees (unidentified); slide reference number AMU/ZD/ NC/Tylencholaimus striatus/1.

\section{Paratypes}

INDIA - 4 우; same collection data as for holotype; slides reference number AMU/ZD/NC/ Tylencholaimus striatus/2-3・1 9 ; same collection data as for holotype; slide reference number AMU/ $\mathrm{ZD} / \mathrm{NC} /$ Tylencholaimus striatus/4, nematode collection of the Zoological Survey of India, Kolkata, India.

\section{Type habitat and locality}

Soil samples collected from around the roots of shrubs and forest trees (unidentified) from Bhagamandala, Karnataka State. 


\section{Description}

Female

Small sized nematodes, slightly curved ventrad to open C-shaped upon fixation; body cylindrical, tapering gradually towards both extremities but more so towards the anterior end. Cuticle with two distinct layers, $1.0-1.5 \mu \mathrm{m}$ thick at midbody and $2.0-2.5 \mu \mathrm{m}$ on tail. Outer cuticle strongly striated; inner layer thick, loose, irregular outline with distinct radial refractive elements. Lateral chords occupying about $26-36 \%$ of midbody diameter. Lateral dorsal and ventral body pores indistinct. Lip region caplike, offset by slight constriction, 1.7-2.0 times as wide as high or about $1 / 3$ to $2 / 5$ of the body diameter at neck base. Lips rounded, amalgamated, inner part slightly elevated. Labial and cephalic papillae distinct but not interfering with the labial contour. Amphids cup-shaped, their aperture occupying about $1 / 3$ to $2 / 5$ of lip region diameter. Stoma a truncate cone. Odontostyle 0.8 times the lip region diameter long, its aperture about $1 / 3$ of the odontostyle length. Odontophore rod-like, as long as odontostyle, with minute basal knobs. Guiding ring simple, refractive, at 0.7-0.9 times the lip region diameter from anterior end. Pharynx consisting of a slender, slightly muscular anterior part, expanding abruptly into a cylindrical basal bulb, with thick-walled lumen, occupying about $36-41 \%$ of total neck length. Pharyngeal gland nuclei and their orifices are located as follows: $\mathrm{DO}=62-66, \mathrm{DN}=65-69, \mathrm{DO}-\mathrm{DN}=2.2-3.1, \mathrm{~S} 1 \mathrm{~N} 1=$ $74-77, \mathrm{~S} 1 \mathrm{~N} 2=78-82, \mathrm{~S} 2 \mathrm{~N}=89-91, \mathrm{~S} 2 \mathrm{O}=91-92$. Nerve ring at $39-44 \%$ of neck length from anterior end. Cardia rounded conoid, about $1 / 3$ of the corresponding body diameter long.

Genital system monodelphic-prodelphic. Ovary reflexed, measuring 29-47 $\mu \mathrm{m}$ long; oocytes arranged in single row except near tip. Oviduct joining the ovary subterminally, measuring $42-54 \mu \mathrm{m}$, its proximal and distal parts not differentiated. Oviduct-uterus junction marked by well-developed sphincter. Uterus short, measuring 19-26 $\mu \mathrm{m}$. Posterior genital branch reduced to a small uterine sac, 5.5-10.0 $\mu \mathrm{m}$ or about $2 / 5$ to $3 / 5$ of the corresponding body diameter long, rarely absent $(n=2)$. Sperm cell not present in the genital tract. Vagina cylindrical, extending inwards, $7.5-9.0 \mu \mathrm{m}$ or about $1 / 2(49-55 \%)$ of midbody diameter; pars proximalis vaginae 5.0-6.0 $\times 2.5-3.5 \mu \mathrm{m}$, encircled by circular muscles; pars distalis vaginae short, 2.5$3.0 \mu \mathrm{m}$ with slightly curved walls; pars refringens absent. Vulva apparently a transverse slit. Prerectum 2.6-4.5 and rectum 1.1-1.5 times anal body diameter long. Tail convex-conoid with bluntly rounded terminus, 1.1-1.3 times anal body diameter long, with a pair of caudal pores on each side.

\section{Male}

Not found.

\section{Taxonomic remarks}

In its small sized body, lip region without labial disc, abrupt pharyngeal expansion, mono-prodelphic gonad and short conoid tail, the new species comes close to T. minutus Vinciguerra, 1986 and T. gallaicus Seijas et al., 2007 but differs from the former in having a comparatively smaller body size (0.30-0.34 vs $0.37-0.52 \mathrm{~mm}$ ); absence of labial disc (vs labial disc present); lower b value (2.5-2.7 vs 2.7-3.5); slightly shorter pharynx (116-126 vs $121-161 \mu \mathrm{m}$ ), shorter pharyngeal expansion (36-41 vs $42-46 \%$ of total neck length); presence of well-developed sphincter (vs sphincter weakly developed); shorter and differently shaped tail (12-15 vs 15-18 $\mu \mathrm{m}$, convex-conoid vs conical with finely rounded terminus) and absence of male (vs present).

The new species differs from $T$. gallaicus in having a posterior vulva position ( $\mathrm{V}=68-72$ vs 60.3-66.7); shorter tail (12-15 vs $20-28 \mu \mathrm{m})$; higher c (23-27 vs $12.3-19.0)$; lower c' (1.1-1.3 vs 1.6-2.4) ratios and absence of male (vs present).

In the presence of monodelphic genital system and conoid tail, the new species comes close to $T$. decens Andrássy, 1991 and T. mirabilis de Man, 1876, but differs from T. decens, in having a comparatively shorter body size $(0.30-0.34$ vs $0.68-0.80 \mathrm{~mm})$; narrower lip region (5.5-6.0 vs 8.0-10 $\mu \mathrm{m})$; lower b 


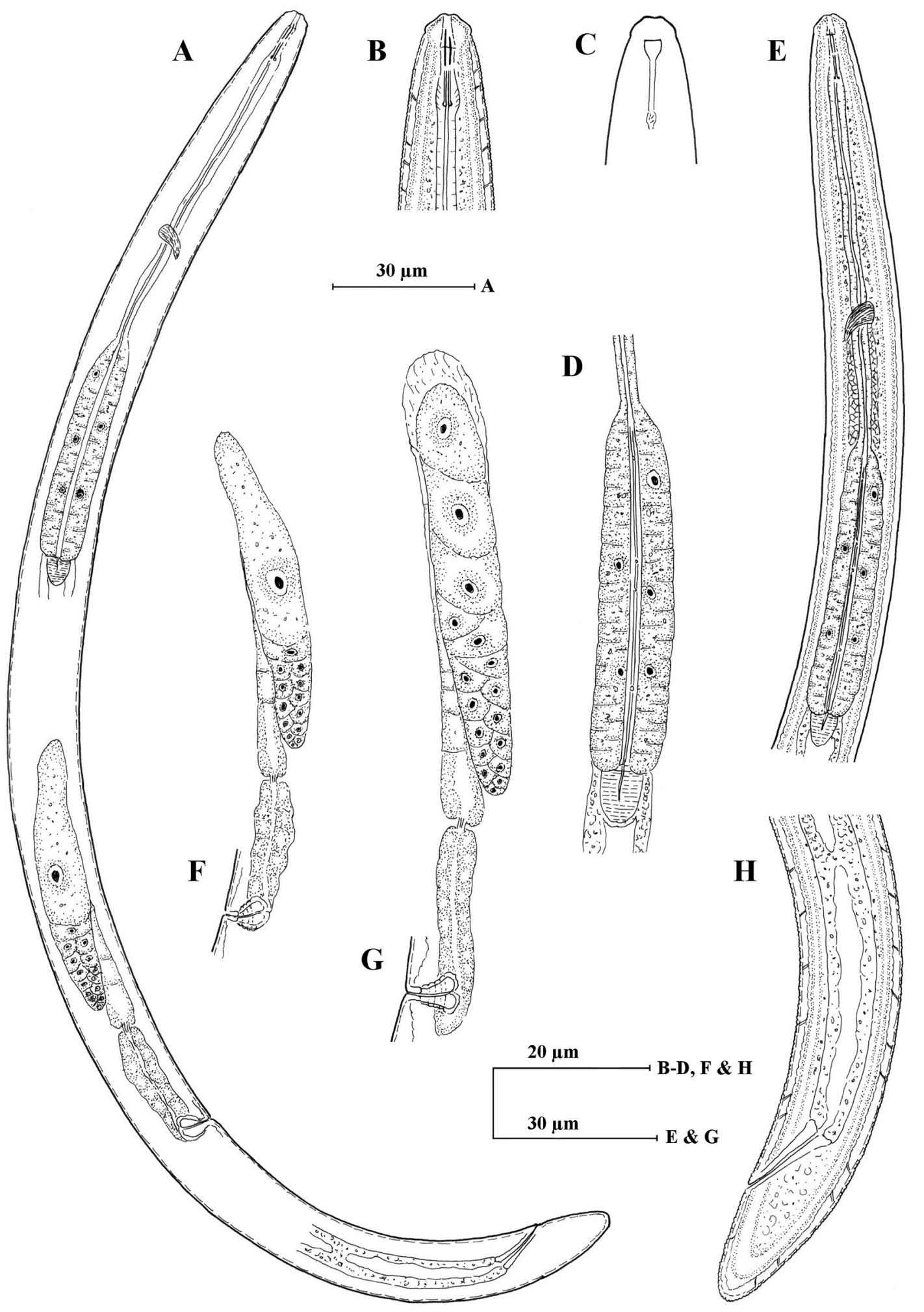

Fig. 13. Tylencholaimus striatus sp. nov., ․ . A, D-F, H. Paratype 1 (slide 2). B-C. Holotype (AMU/ $\mathrm{ZD} / \mathrm{NC} /$ Tylencholaimus striatus/1). G. Paratype 5 (slide 4). A. Entire specimen. B. Anterior region C. Anterior region showing amphid. D. Expanded part of pharynx. E. Pharyngeal region. F-G. Genital system. H. Posterior region. 

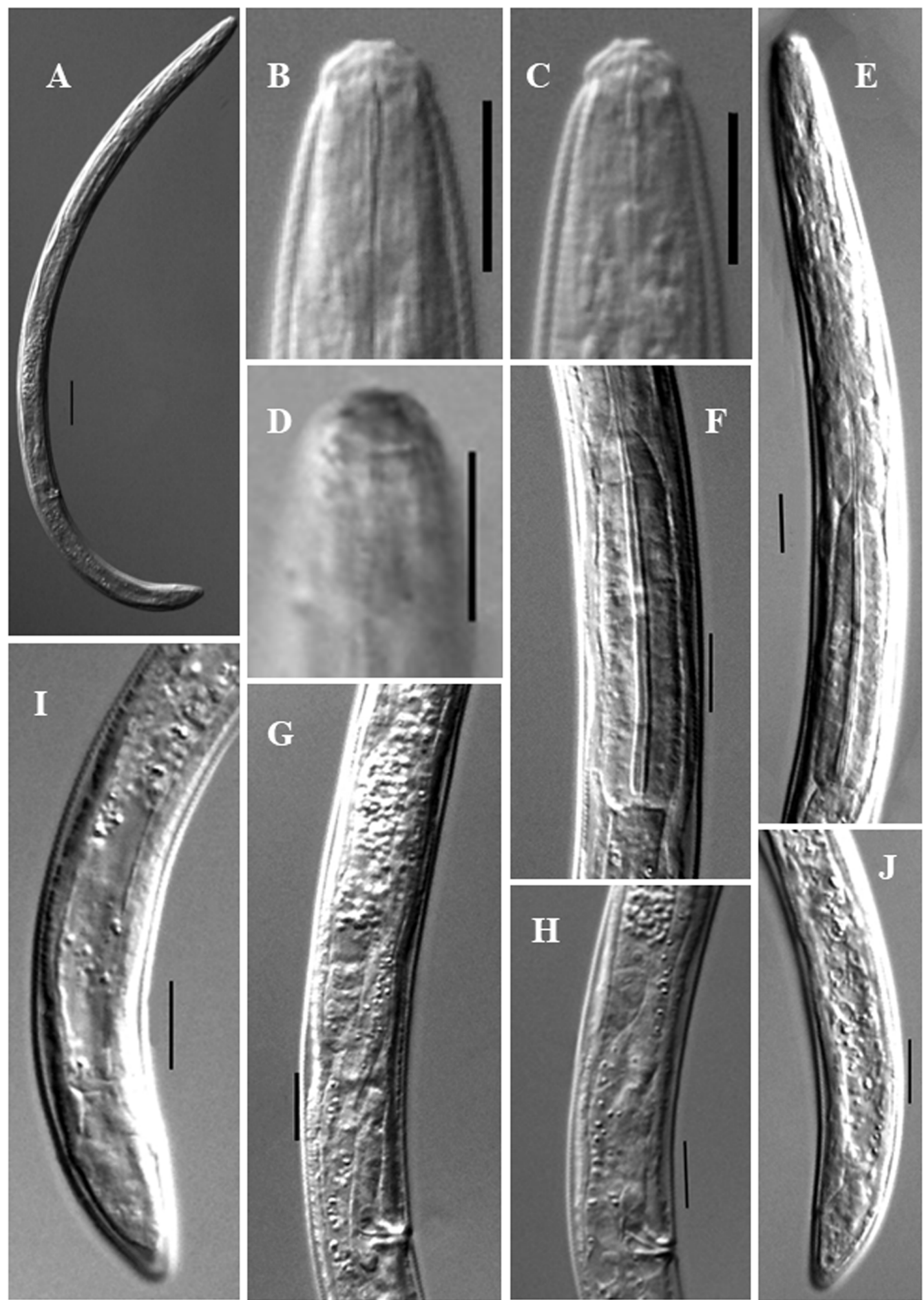

Fig. 14. Tylencholaimus striatus sp. nov., $q$ (LM photographs). A, C, I. Paratype 1 (slide 2). B, H. Holotype (AMU/ZD/NC/Tylencholaimus striatus/1). D. Paratype 2, (slide 2). E, G. Paratype 5 (slide 4). F. Paratype 3 (slide 3). J. Paratype 4, (slide 3). A. Entire specimen. B-C. Anterior region. D. Anterior region showing amphid. E. Pharyngeal region. F. Expanded part of pharynx. G-H. Genital system. I-J. Posterior region. Scale bars: A $=20 \mu \mathrm{m}$; B-J $=10 \mu \mathrm{m}$. 
Table 9. Measurements of Tylencholaimus striatus sp. nov. All measurements are in $\mu \mathrm{m}$ and in the form: mean \pm s.d. (range).

\begin{tabular}{|c|c|c|}
\hline Characters & Holotype female & Paratypes females \\
\hline $\mathrm{n}$ & & 5 \\
\hline $\mathrm{L}$ & 338 & $326.1 \pm 13.9(309-349)$ \\
\hline $\mathrm{a}$ & 22.5 & $20.9 \pm 0.70(20.2-22.3)$ \\
\hline $\mathrm{b}$ & 2.6 & $2.6 \pm 0.07(2.5-2.7)$ \\
\hline $\mathrm{c}$ & 26 & $24.7 \pm 1.27(23-27.3)$ \\
\hline$c^{\prime}$ & 1.3 & $1.12 \pm 0.04(1.1-1.3)$ \\
\hline $\mathrm{V}$ & 68.7 & $71.1 \pm 0.92(69.9-72.5)$ \\
\hline G1 & 25.7 & $26.1 \pm 1.2(25.2-28.3)$ \\
\hline G2 & 1.7 & $2.1 \pm 0.50(1.7-2.9)$ \\
\hline Body diameter at neck base & 15 & $15.1 \pm 0.43(14.5-15.5)$ \\
\hline Body diameter at mid body & 15 & $15.2 \pm 0.48(14.5-15.5)$ \\
\hline Body diameter at anus & 10 & $10.5 \pm 0.73(10-12)$ \\
\hline Lip region diameter & 6.0 & $5.7 \pm 0.2(5.5-6.0)$ \\
\hline Lip region height & 3.0 & $3.2 \pm 0.18(3.0-3.5)$ \\
\hline Amphidial aperture & 2.0 & $2.01 \pm 0.19(2.0-2.5)$ \\
\hline Odontostyle length & 5.0 & $5.2 \pm 0.19(5.0-5.5)$ \\
\hline Odontophore length & 5.0 & $5.1 \pm 0.23(5.0-5.5)$ \\
\hline Total stylet length & 10 & $10.1 \pm 0.39(10-11)$ \\
\hline Guiding ring from anterior end & 4.0 & $4.6 \pm 0.54(4.0-5.5)$ \\
\hline Nerve ring from anterior end & 52 & $52.9 \pm 3.8(46-56)$ \\
\hline Neck length & 126 & $121.5 \pm 3.5(116-126)$ \\
\hline Expanded part of pharynx & 49 & $48.4 \pm 1.5(46-51)$ \\
\hline Cardia length & 5.0 & $5.1 \pm 0.73(4.0-6.0)$ \\
\hline Anterior genital branch & 87 & $85.4 \pm 3.7(80-91)$ \\
\hline Posterior genital branch & 6.0 & $7.1 \pm 1.84(5.5-10)$ \\
\hline Vaginal length & 7.5 & $7.9 \pm 0.48(7.0-9.0)$ \\
\hline Vulva from anterior end & 237 & $232.8 \pm 12.4(216-253)$ \\
\hline Prerectum length & 31 & $38.2 \pm 4.6(31-45)$ \\
\hline Rectum length & 12 & $11.3 \pm 0.99(10-13)$ \\
\hline Tail length & 13 & $12.9 \pm 0.96(12-15)$ \\
\hline
\end{tabular}


value $(2.5-2.7$ vs $3.2-3.4)$; shorter odontostyle $(5.0-5.5$ vs $7.0-8.0 \mu \mathrm{m})$ and odontophore $(5.0-5.5 \mathrm{vs}$ 8.0-9.0 $\mu \mathrm{m})$; shorter pharynx (116-126 vs 207-232 $\mu \mathrm{m})$; shorter pharyngeal bulb (46-51 vs 99-115 $\mu \mathrm{m})$; shorter tail (12-15 vs 19-21 $\mu \mathrm{m})$, lower c (23-27 vs 31-38) and slightly higher c' (1.1-1.3 vs 0.9-1.1) ratios.

The new species differs from T. mirabilis in having a smaller body size $(0.30-0.34$ vs $0.69-1.0 \mathrm{~mm})$, narrower lip region $(5.5-6.0$ vs $8.0-10 \mu \mathrm{m})$; lower b value (2.5-2.7 vs 2.9-4.4); shorter odontostyle (5.0-5.5 vs 6.0-7.0 $\mu \mathrm{m})$ and odontophore (5.0-5.5 vs 8.5-11.5 $\mu \mathrm{m})$; shorter pharynx (116-126 vs 186$254 \mu \mathrm{m})$, shorter pharyngeal expansion (46-51 vs $88-112 \mu \mathrm{m})$; shorter tail (12-15 vs 24-34 $\mu \mathrm{m})$ and absence of male (vs present).

\section{Tylencholaimus tamiliensis sp. nov. \\ urn:1sid:zoobank.org:act:90F61B5B-B85A-4BD0-823F-76A964973D92}

Figs 15-16, Table 10

\section{Diagnosis}

Tylencholaimus tamiliensis sp. nov. is characterized by its small slender body, $0.51-0.58 \mathrm{~mm}$ long; lip region cap-like, offset, lips elevated; odontostyle slender 5.5-6.0 $\mu \mathrm{m}$, odontophore 6.0-6.5 $\mu \mathrm{m}$, total stylet length $11.5-12.5 \mu \mathrm{m}$; pharynx with slightly muscular anterior part, expanding gradually into the cylindrical basal bulb occupying about $40-43 \%$ of total neck length; female genital system monodelphicprodelphic; posterior genital branch reduced to a simple sac, 1.3-2.0 times midbody diameter long; vulva a transverse slit and tail short, rounded to conoid with sunken terminus.

\section{Etymology}

The new species is named Tylencholaimus tamiliensis sp. nov. because of its type locality Tamil Nadu.

\section{Material examined}

\section{Holotype}

INDIA • 1 P; Tamil Nadu State, Nilgiris district, Naduvattum; $11^{\circ} 28^{\prime} 37.8^{\prime \prime}$ N, 76 $36^{\prime \prime} 36.7^{\prime \prime}$ E; 5-15 cm depth; 15 Nov. 2016; roots of shrubs (unidentified); slide reference number AMU/ZD/NC/Tylencholaimus tamiliensis/1.

\section{Paratypes}

INDIA - 6 우; same collection data as for holotype; slides reference number AMU/ZD/NC/ Tylencholaimus tamiliensis/2-3 -3 우; same collection data as for holotype; slide reference number AMU/ZD/NC/Tylencholaimus tamiliensis/4, nematode collection of the Zoological Survey of India, Kolkata, India.

\section{Type habitat and locality}

Soil samples collected from around the roots of shrubs (unidentified) from forest near Naduvattum, Nilgiris district, Tamil Nadu State.

\section{Description}

\section{Female}

Moderately sized nematodes, slightly curved ventrad upon fixation; body cylindrical, tapering gradually towards both extremities but more so towards the anterior end. Cuticle with two distinct layers, 1.5$2.0 \mu \mathrm{m}$ thick at midbody and $2.5-3.0 \mu \mathrm{m}$ on tail. Outer cuticle with fine transverse striations; inner layer thick, its outline loose irregular, with distinct radial refractive elements. Lateral chords occupying about $22-31 \%$ of midbody diameter. Lateral body pores indistinct, dorsal body pores one at odontostyle- 
odontophore region, 1-2 in neck region, one between pharyngeal base to vulva and one at post-vulval region; ventral body pores: 1-2 in neck region, and 2 in post-vulval region. Lip region cap-like, offset by deep constriction, 2.0-2.3 times as wide as high or about $1 / 3$ of the body diameter at neck base. Lips rounded, amalgamated, inner part slightly elevated. Labial and cephalic papillae distinct but not interfering with the labial contour. Amphids small, cup-shaped, their aperture occupying about $1 / 3$ of lip region diameter. Stoma a truncate cone. Odontostyle $0.7-0.9$ times the lip region diameter long, its aperture about $1 / 4$ to $1 / 5$ of the odontostyle length. Odontophore rod-like, with minute basal knobs, about 1.0-1.1 times the odontostyle length. Guiding ring simple, refractive, at $0.6-0.8$ times the lip region diameter from anterior end. Pharynx consisting of a slightly muscular anterior part, expanding gradually into a cylindrical basal bulb, with thick-walled lumen, occupying about $40-43 \%$ of total neck length. Pharyngeal gland nuclei and their orifices are located as follows: $\mathrm{DO}=58-62, \mathrm{DN}=61-64, \mathrm{DO}-\mathrm{DN}=2.1-3.5, \mathrm{~S} 1 \mathrm{~N} 1=74-77$, $\mathrm{S} 1 \mathrm{~N} 2=77-82, \mathrm{~S} 2 \mathrm{~N}=87-89, \mathrm{~S} 2 \mathrm{O}=89-92$. Nerve ring located at $37-41 \%$ of neck length from anterior region. Cardia rounded conoid, about $1 / 3$ to $2 / 5$ of the corresponding body diameter long.

Genital system monodelphic-prodelphic. Ovary reflexed, measuring 48-70 $\mu \mathrm{m}$ long; oocytes arranged in single row except near tip. Oviduct joining the ovary subterminally, measuring $52-83 \mu \mathrm{m}$, consisting of a slender distal part and well developed pars dilatata. Oviduct-uterus junction marked by welldeveloped sphincter. Uterus short, tubular, measuring 32-43 $\mu \mathrm{m}$. Posterior genital branch reduced to a short uterine sac, 1.3-2.0 times the corresponding body diameter. Sperm cell not present in the genital tract. Vagina cylindrical, $10-12 \mu \mathrm{m}$ or about $2 / 5$ to $1 / 2$ (44-53\%) of midbody diameter; pars proximalis vaginae 7.0-8.0 × 5.0-6.5 $\mu \mathrm{m}$, its wall encircled by circular muscles; pars distalis vaginae 3.0-4.0 $\mu \mathrm{m}$ with slightly curved walls; pars refringens absent. Vulva a transverse slit. Prerectum 3.0-5.4 and rectum 1.0-1.3 times anal body diameter long. Tail short, rounded to convex-conoid with sunken terminus, 0.7-0.9 times anal body diameter long, with a dorsal pore at level of anal opening and a pair of subdorsal caudal pores.

\section{Male \\ Not found.}

\section{Taxonomic remarks}

In the absence of labial disc, gradual pharyngeal expansion, presence of posterior uterine sac and short tail with sunken terminus, the new species comes close to T. australis Yeates, 1979; T. orientalis Li et al., 2008; T. maritus Loof \& Jairajpuri, 1968 and T. loofi Dhanachand, 1994 but differs from the former in having a distinctly offset lip region, with elevated, angular and slightly separate lips (vs not elevated, flat, rounded and amalgamated), slightly higher lip region (3.0-3.5 vs 2.5-3.0 $\mu \mathrm{m}$ ); body pores distinct (vs indistinct), slightly higher c (39.7-48.8 vs 36-40), lower c' (0.7-0.9 vs 1.0-1.1) ratios and presence of three caudal pores (vs two caudal pores).

It differs from $T$. orientalis in having a slightly smaller pharyngeal expansion (40-43 vs $44-45 \%$ of total neck length); longer post-uterine sac (30-44 vs 10-27 $\mu \mathrm{m}$ ); tail short, rounded to conoid with sunken tip (vs rounded tip) and presence of three caudal pores (vs two caudal pores).

The new species differs from T. maritus in having a comparatively posterior vulva position (V $=68-$ 72 vs 63-66); longer post-uterine sac (30-44 vs 6.0-9.5 $\mu \mathrm{m})$; lower c' ratio $(0.7-0.9$ vs $1.0-1.3)$; tail short, rounded to conoid with sunken tip (vs rounded tip); caudal pores three (vs two) and absence of male (vs present).

The new species differs from $T$. loof in having a shorter total stylet length $(11.5-12.5$ vs $22.4-25.6 \mu \mathrm{m})$; slightly posterior vulva position ( $\mathrm{V}=68-72 \mathrm{vs} 65.0-67.5)$; longer post-uterine sac (30-44 vs $10 \mu \mathrm{m})$; longer prerectum ( $42-80$ vs $24-25 \mu \mathrm{m}$ ) and tail with sunken tip (vs conoid tip). 

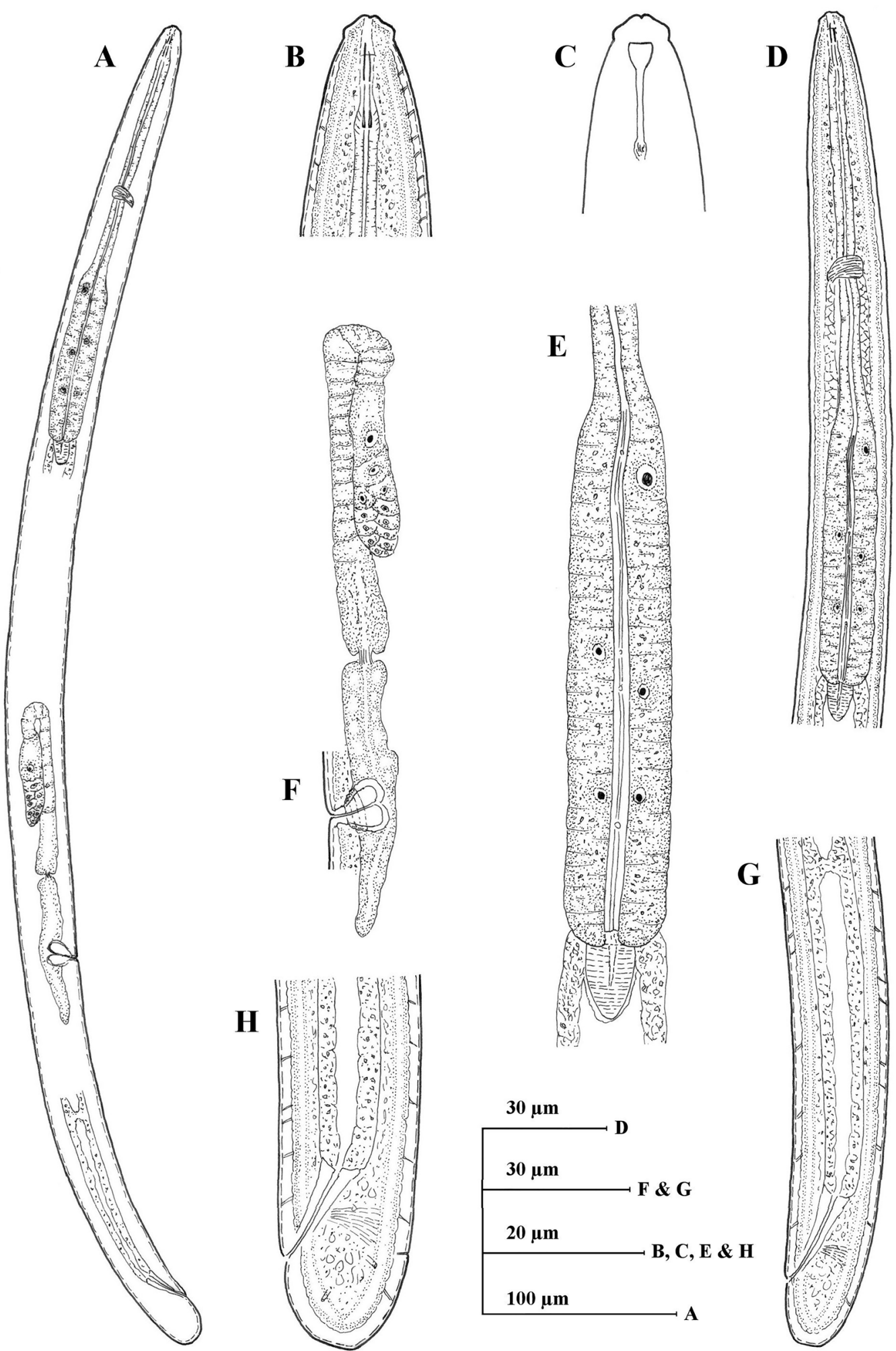
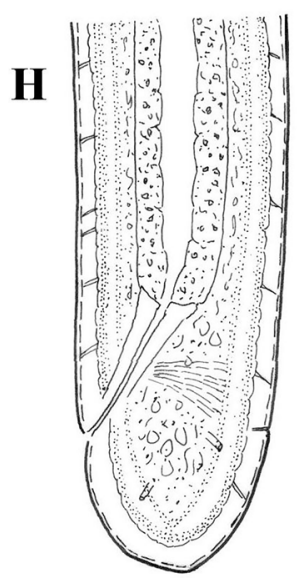
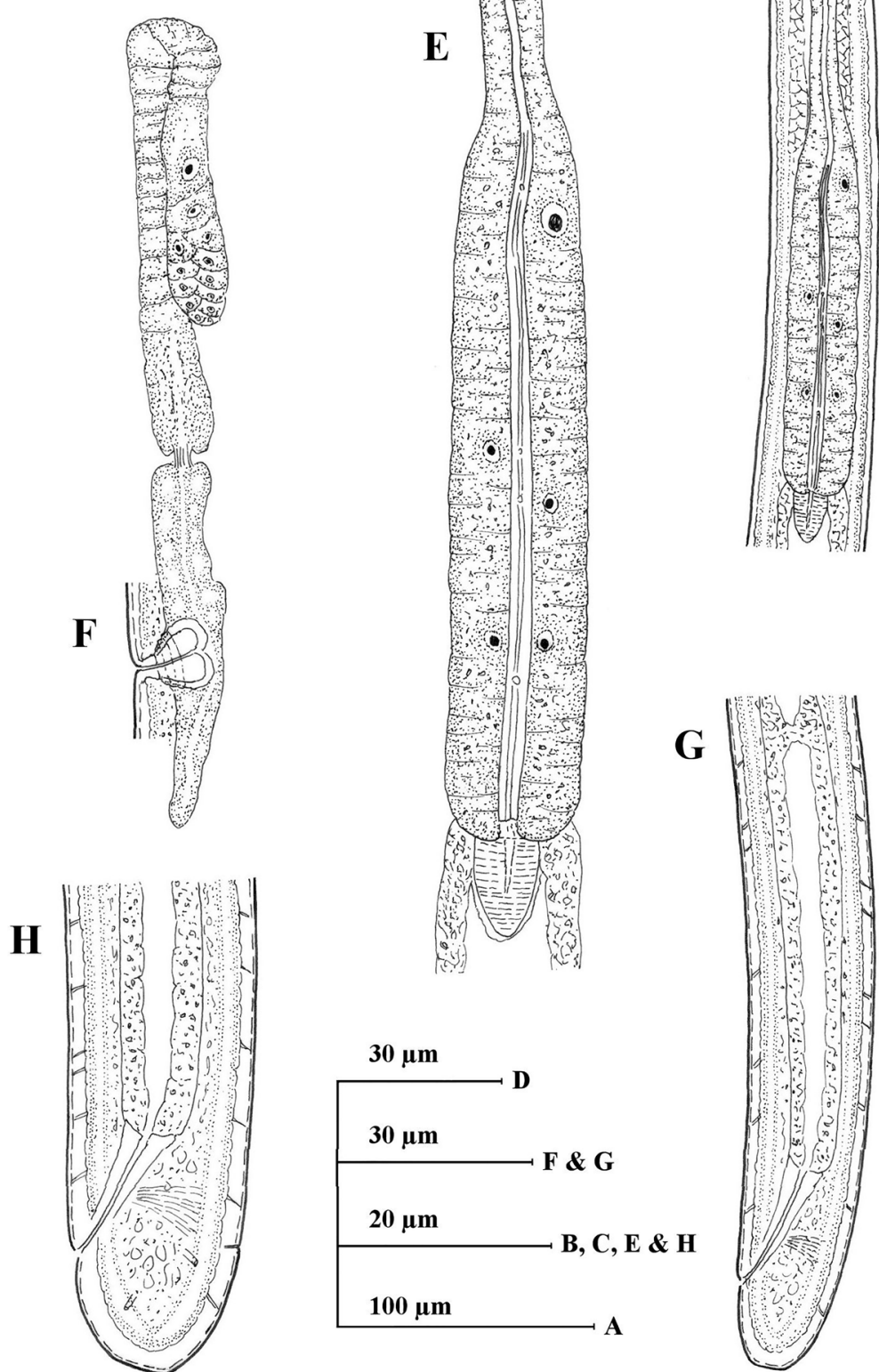

Fig. 15. Tylencholaimus tamiliensis sp. nov., 9 . A, C-F. Paratype 5 (slide 3). B. Paratype 2 (slide 2). G-H. Holotype (AMU/ZD/NC/Tylencholaimus tamiliensis/1). A. Entire specimen. B. Anterior region. C. Anterior region showing amphid. D. Pharyngeal region. E. Pharyngeal expansion. F. Genital system. G-H. Posterior region. 

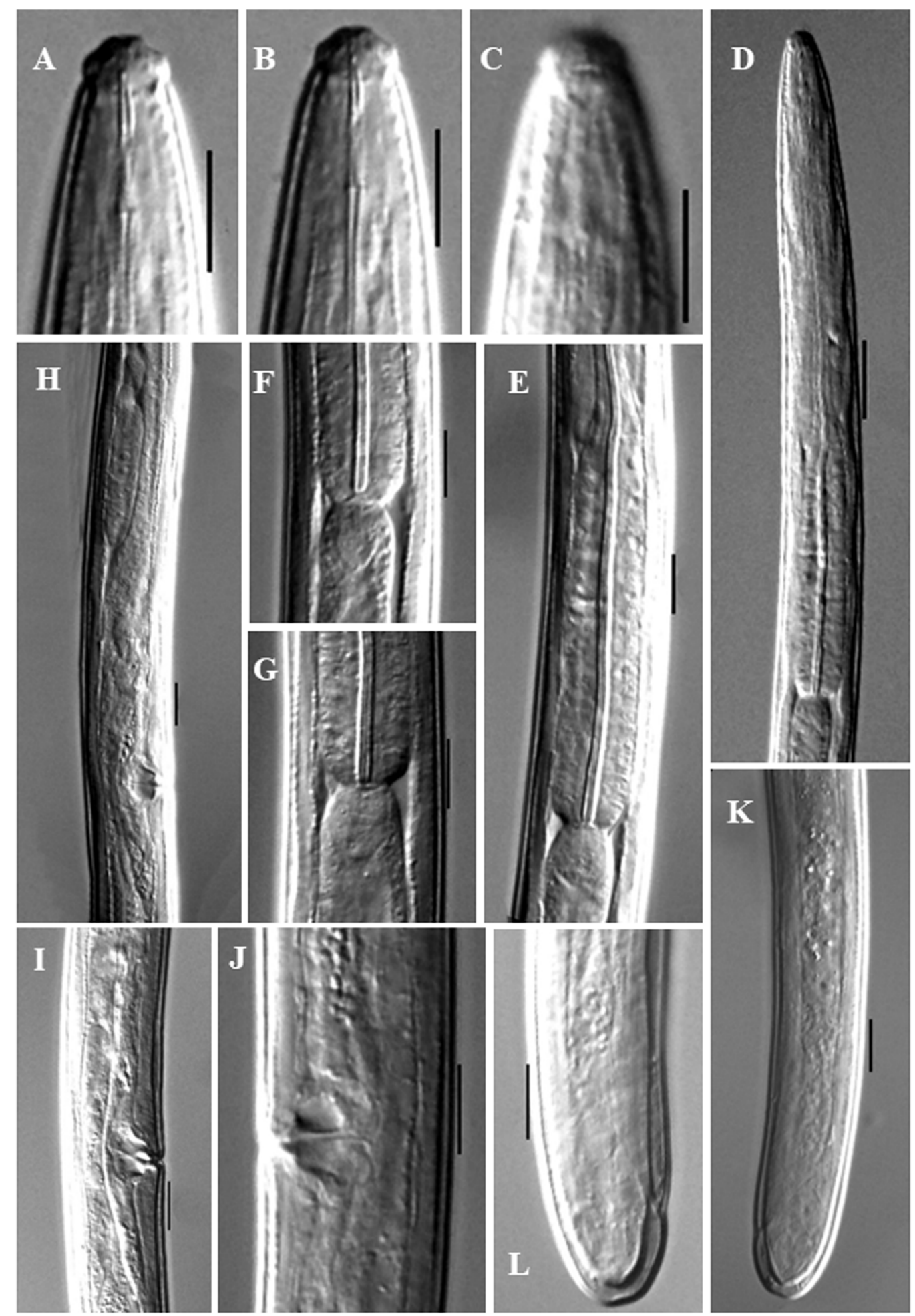

Fig. 16. Tylencholaimus tamiliensis sp. nov., + (LM photographs). A, F. Paratype 1, (slide 2). B, G, J. Paratype 2 (slide 2). C. Paratype 5 (slide 3). D, I. Paratype 4 (slide 3). E. Paratype 8 (slide 4). H. Paratype 6 (slide 3). K-L. Holotype (AMU/ZD/NC/Tylencholaimus tamiliensis/1). A-B. Anterior region. C. Anterior region showing amphid. D. Pharyngeal region. E. Expanded part of pharynx. F-G. Pharyngo-intestinal junction. H-I. Genital system. J. Vulval region. K. Posterior region. L. Posterior end. Scale bars: A-C, E-G, I-L $=10 \mu \mathrm{m}$; D, H $=20 \mu \mathrm{m}$. 
Table 10. Measurements of Tylencholaimus tamiliensis sp. nov. All measurements are in $\mu \mathrm{m}$ and in the form: mean \pm s.d. (range).

\begin{tabular}{|c|c|c|}
\hline Characters & Holotype female & Paratypes females \\
\hline $\mathrm{n}$ & & 9 \\
\hline $\mathrm{L}$ & 580 & $541.6 \pm 19.9(511-574)$ \\
\hline a & 25.7 & $24.6 \pm 1.1(24.0-27.9)$ \\
\hline $\mathrm{b}$ & 3.3 & $3.1 \pm 0.15(3.0-3.4)$ \\
\hline $\mathrm{c}$ & 45.6 & $44.3 \pm 2.8(39.7-48.8)$ \\
\hline$c^{\prime}$ & 0.81 & $0.80 \pm 0.06(0.7-0.9)$ \\
\hline $\mathrm{V}$ & 70.8 & $68.4 \pm 1.2(68-72)$ \\
\hline G1 & 24.2 & $21.3 \pm 1.2(18-22)$ \\
\hline G2 & 5.4 & $6.4 \pm 0.75(5.5-7.5)$ \\
\hline Body diameter at neck base & 23 & $22.5 \pm 0.65(21.5-23.5)$ \\
\hline Body diameter at mid body & 23 & $21.5 \pm 0.65(20.5-22.5)$ \\
\hline Body diameter at anus & 15.5 & $14.9 \pm 0.69(14-16)$ \\
\hline Lip region diameter & 7.5 & $7.2 \pm 0.40(7.0-8.0)$ \\
\hline Lip region height & 3.5 & $3.2 \pm 0.24(3.0-3.5)$ \\
\hline Amphidial aperture & 2.5 & $2.3 \pm 0.15(2.0-2.5)$ \\
\hline Odontostyle length & 6.0 & $5.6 \pm 0.24(5.5-6.0)$ \\
\hline Odontophore length & 6.5 & $6.2 \pm 0.20(6.0-6.5)$ \\
\hline Total stylet length & 12.5 & $11.8 \pm 0.30(11.5-12.5)$ \\
\hline Guiding ring from anterior end & 5.0 & $5.4 \pm 0.42(5.0-6.0)$ \\
\hline Nerve ring from anterior end & 63 & $65.3 \pm 4.1(60-73)$ \\
\hline Neck length & 172 & $168.3 \pm 6.6(156-179)$ \\
\hline Expanded part of pharynx & 73 & $70.7 \pm 3.6(64-76)$ \\
\hline Cardia length & 10 & $9.8 \pm 0.92(9.0-12)$ \\
\hline Anterior genital branch & 144 & $118.2 \pm .9(100-129)$ \\
\hline Posterior genital branch & 32 & $35.4 \pm 5.1(30-44)$ \\
\hline Vaginal length & 12 & $10.8 \pm 0.74(10-12)$ \\
\hline Vulva from anterior end & 411 & $378.3 \pm 17.5(351-412)$ \\
\hline Prerectum length & 66 & $58.2 \pm 13.0(42-80)$ \\
\hline Rectum length & 19 & $16.9 \pm 1.7(15-21)$ \\
\hline Tail length & 13 & $12.2 \pm 0.86(11-14)$ \\
\hline
\end{tabular}


The new species also comes close to T. chathami Yeates, 1979 but differs in having a comparatively shorter body length $(0.51-0.58$ vs $0.6-0.8 \mathrm{~mm})$; shorter pharynx and its expansion (156-179 vs 192$223 \mu \mathrm{m}, 64-76$ vs $84-103 \mu \mathrm{m})$; longer post-uterine sac (1.3-2.0 vs 0.25 times the corresponding body diameter long); tail with sunken tip (vs rounded tip) and three caudal pores (vs two).

\section{Discussion}

The genus Tylencholaimus is one of the most widely distributed and speciose taxa under the superfamily Tylencholaimoidea of the order Dorylaimida. Its representatives generally occur in undisturbed natural soils, predominantly recorded from temperate Northern Hemisphere i.e., North America and Eurasia (Golhasan et al. 2019). A large number of species have been reported from India, representing about $40 \%$ of its total species. The Western Ghats in India constitutes a range of tropical rainforests with a very high degree of species richness and endemism. Only three species, representing the genus Tylencholaimus have so far been recorded from this region by Dhanam \& Jairajpuri (1999). In the course of the present study, several populations of this genus were recorded from soil samples collected from different habitats and localities. The presently reported populations represented five known species (T. mirabilis, T. teres, T. micronanus, T. ibericus and T. cosmos) and five new species. With the addition of the presently described new species, the total number of valid species under this genus is raised to 62 (Ahad \& Ahmad 2016; Wu et al. 2018; Golhasan et al. 2019), 27 of them are recorded from India, and 11 species are recorded from the Western Ghats. Tylencholaimus mirabilis, the type species is reported here for the first time from India. Two known species T. micronanus and T. teres are reported here for the first time from the Western Ghats and a male specimen was also described for T. ibericus. As a result of this survey, the Western Ghats comprises 11 species of the genus Tylencholaimus, which constitutes about $40 \%$ of the genus diversity in India and 17\% of the World's Tylencholaimus diversity.

\section{Acknowledgments}

The first author thanks the University Grants Commission for a UGC-Non-Net Fellowship. The authors are also thankful to the DST-PURSE programme for financial assistance.

\section{References}

Ahad S. \& Ahmad W. 2016. Description of two new and six known species of the genus Tylencholaimus de Man, 1876 (Nematoda: Dorylaimida) with a diagnostic compendium and key to species. Zootaxa 4107: 451-490. https://doi.org/10.11646/zootaxa.4107.4.1

Ahmad W. 1993. Description of Thonus goaensis sp. n. and Coomansinema oryzae sp n. (Nematoda: Dorylaimida). Afro-Asian Journal of Nematology 3: 173-176.

Ahmad A. \& Ahmad W. 2002. Descriptions of three new species of Dorylaimoidea (Nematoda: Dorylaimida) from South India. International Journal of Nematology 12: 23-28.

Ahmad M. \& Jairajpuri M.S. 1979. Four new species of Leptonchidae (Nematoda: Dorylaimida). Indian Journal of Nematology 9: 125-135.

Ahmad W. \& Ahmad I. 1992. Makatinus heynsi n. sp. (Dorylaimida: Aporcelaimidae) from Goa, India. Fundamental and Applied Nematology 15: 149-152.

Ahmad W. \& Araki M. 2003. New and known species of the family Tylencholaimidae (Nematoda: Dorylaimida) from Japan. Journal of Nematode Morphology and Systematics 6: 1-26.

Ahmad W. \& Jairajpuri M.S. 1982. Studies on the genus Axonchium Cobb, 1920 from India. Nematologica 28: 21-33. https://doi.org/10.1163/187529282X00484 
Ahmad W. \& Jairajpuri M.S. 1983. Two new species of the genus Iotonchus (Nematoda: Mononchida) from Silent Valley, Kerala, India. Systematic Parasitology 5: 83-87. https://doi.org/10.1007/BF00049236

Ahmad W. \& Jairajpuri M.S. 1984. Two new species of Dorylaimoidea (Nematoda: Dorylaimida) from Goa, India. Revue de Nématologie 7: 393-397.

Ahmad W. \& Jairajpuri M.S. 1986. Silvallis sclerostoma n. gen., n. sp. (Nematoda: Dorylaimida) from Silent Valley, Kerala, India. Nematologica 31: 375-378. https://doi.org/10.1163/187529285X00481

Ahmad W. \& Jairajpuri M.S. 1988. Some studies on Lenonchium (Nematoda: Dorylaimida) with description of L. macrodorum n. sp. Revue de Nématologie 11: 7-11

Ahmad W., Ahmad I., Bilgrami A.L. \& Khan Z. 1992. Thalassogenus shamimi n. sp. (Nematoda: Thalassogeneridae), a nematode predator from India. Fundamental and Applied Nematology 15: 127-131.

Ali S.M. \& Chisty K.Z. 1972. Tylencholaimus suryawnshii n. sp. (Nematoda: Tylencholaimidae) from Marathwada, India. Marathwada University Journal of Science, Section B (Biological Sciences) 11: 283-285.

Baqri Q.H. 1991. Contribution to the fauna of Sikkim. Nematodes associated with citrus from Sikkim, India. Records of the Zoological Survey of India (occassional paper) 128: 1-103.

Bütschli O. 1873. Beiträge zur Kenntnis der freilebenden Nematoden. Nova acta Academiae Caesareae Leopoldino-Carolinae Germanicae Naturae Curiosorum 36: 1-124. Available from https://www.biodiversitylibrary.org/page/12601002 [accessed 11 Aug. 2021].

CEPF 2007. Ecosystem Profile: Western Ghats and Sri Lanka Biodiversity Hotspot, Western Ghats Region.

Cobb N.A. 1918. Estimating the nema population of the soil. United States Department of Agriculture, Bureau of Plant Industry, Agriculture Technical Circular 1: 1-48.

Coomans A. 1962. Some species of Dorylaimoidea found in Belgium. I. Members of the Tylencholaiminae Filipjev, 1934. Nematologica 7: 146-154. https://doi.org/10.1163/187529262X00882

De Maeseneer J. \& d'Herde J. 1963. Méthodes utilisées pour l'étude des anguillules libres du sol. Revue d'Agriculture Bureaux 16: 441-447.

De Man J.G. 1876. Onderzoekingen over vrij in de aarde levende Nematoden. Tijdschrift der Nederlandsche Dierkundige Vereeniging 2: 78-196. Available from https://www.biodiversitylibrary.org/page/9771869 [accessed 11 Aug. 2021].

De Man J.G. 1880. Die einheimischen, frei in der reinen Erde und im süssen Wasser lebende Nematoden. Tijdschrift der Nederlandsche Dierkundige Vereeniging 5: 1-104. Available from https://www.biodiversitylibrary.org/page/9964525 [accessed 11 Aug. 2021].

De Man J.G. 1884. Die, frei in der reinen Erde und im süssen Wasser lebenden Nematoden der niederländischen Fauna: eine systematisch-faunistische Monographie. Brill, Leiden. Available from https://archive.org/details/diefreiinderrein00manj [accessed 11 Aug. 2021].

Dhanachand C. 1994. Tylencholaimus loofi nomen novum for T. minutus Dhanachand et al., 1992. Current Nematology 4: 243.

Dhanam M. \& Jairajpuri M.S. 1999. New leptonchid nematodes: one new genus and eleven new species from Malnad tracts of Karnataka, India. International Journal of Nematology 9: 205-209.

Ferris V.R., Goseco C.G. \& Kumar A.C. 1979. Proleptonchoides southindiae n. gen., n. sp., a new leptonchid from south India. Journal of Nematology 11: 70-72. 
Golhasan B., Heydari R. \& Peña-Santiago R. 2019. Description of Tylencholaimus discus sp. n. (Nematoda, Dorylaimida, Tylecholaimidae) from Iran. Zootaxa 4551: 379-384.

https://doi.org/10.11646/zootaxa.4551.3.6

Jairajpuri M.S. 1965. Three new species of the genus Tylencholaimus De Man, 1876 (Nematoda: Dorylaimoidea) from India. Nematologica 10: 515-518. https://doi.org/10.1163/187529264X00187

Khan E. \& Laha S.K. 1982. Seven new dorylaimid nematodes from Indian Agricultural Research Institute farm, Delhi, India. Indian Journal of Nematology 12: 246-248.

Khan T.H.\& Ahmad W. 1994. Descriptions of Ischiodorylaimus paraugandanus sp. n. and Tylencholaimus asymmetricus sp. n. (Nematoda: Dorylaimida) from India. Indian Journal of Nematology 24: 83-87.

Khan T.H., Jairajpuri M.S. \& Ahmad W. 1989. Descriptions of some new and known species of dorylaim nematodes. Nematologica 35: 419-437. https://doi.org/10.1163/002825989X00179

LiY., Baniyamuddin M., Ahmad W. \& Wu J. 2008. Four new and four known species of Tylencholaimoidea (Dorylaimida: Nematoda) from China. Journal of Natural History 42: 1991-2010.

https://doi.org/10.1080/00222930802254722

Loof P.A.A. 1961. The nematode collection of Dr. J. G. de Man 1. Beaufortia 8: 169-254. Available from https://repository.naturalis.n1/pub/504815 [accessed 11 Aug. 2021].

Loof P.A.A. \& Coomans A. 1970. On the development and location of the oesophageal gland nuclei in the Dorylaimina. Proceedings of the IX International Nematology Symposium, Warsaw 1967, Poland: $79-161$.

Loof P.A.A. \& Jairajpuri M.S. 1968. Taxonomic studies on the genus Tylencholaimus De Man, 1876 (Dorylaimoidea) with a key to the species. Nematologica 14: 317-350.

https://doi.org/10.1163/187529268X00011

Meyl A.H. 1956. Beiträge zur freilebenden nematodenfauna Brasiliens, I. Achth eue nematodenarten der Überfamilie Dorylaimoidea. Nematologica 1: 311-325. https://doi.org/10.1163/187529256X00320

Mohilal N. \& Dhanachand C. 2000. Studies on soil nematodes of Manipur-VII: Tylencholaimus vanguimus sp. n. and Dorella papilla sp. n. Indian Journal of Nematology 30: 33-37.

Mohilal N. \& Dhanachand C. 2003. Studies on soil nematodes of Manipur-XI: two new and one known species of Tylencholaimus. Uttar Pradesh Journal of Nematology 23: 87-92.

Mushtaq P., Naz T. \& Ahmad W. 2007. Descriptions of new and known species of the superfamily Tylencholaimoidea (Nematoda: Dorylaimida) from Jammu \& Kashmir. India. Journal of Nematode Morphology and Systematics 10: 11-29.

Myers N., Mittermeier R.A., Mittermeier C., da Fonseca G.A.B \& Kent J. 2000. Biodiversity hotspots for conservation priorities. Nature 24: 853-857. https://doi.org/10.1038/35002501

Peña-Santiago R. 2008. The genus Tylencholaimus de Man, 1876 (Dorylaimida: Tylencholaimidae) revisited twelve years after. Journal of Nematode Morphology and Systematics 11: 119-128.

Peña-Santiago R. 2020. On the identity of some dorylaims (Dorylaimida) recently described from China. Nematology 22: 957-960. https://doi.org/10.1163/15685411-bja10043

Peña-Santiago R. \& Coomans A. 1994a. Revision of the genus Tylencholaimus de Man, 1876. Didelphic species. Nematologica 40: 32-68. https://doi.org/10.1163/003525994X00049

Peña-Santiago R \& Coomans A. 1994b. Revision of the genus Tylencholaimus de Man, 1876. Prodelphic species: Part II. Nematologica 40: 186-213. https://doi.org/10.1163/003525994X00139 
Peña-Santiago R \& Coomans A. 1994c. Revision of the genus Tylencholaimus de Man, 1876. Prodelphic species: Part III. Nematologica 40: 348-368. https://doi.org/10.1163/003525994X00256

Rahman M.F., Jairajpuri M.S., Ahmad W. \& Ahmad I. 1987. Three new species of dorylaim nematodes from the North-Eastern region of India. Indian Journal of Nematology 16: 197-204.

Seinhorst J.W. 1959. A rapid method for the transfer of nematodes from fixative to anhydrous glycerine. Nematologica 15: 81-100. https://doi.org/10.1163/187529259X00381

Tabinda N., Anjum A. \& Ahmad W. 2013. Mononchida (Nematoda) from Silent Valley National Park, India. Zootaxa 3635 (3): 224-236. https://doi.org/10.11646/zootaxa.3635.3.2

Tarjan A.C. 1953. Known and suspected plant-parasitic nematodes of Rhode Island, I. Proceedings of the Helminthological Society of Washington 20: 49-54.

Tarjan A.C. 1956. Known and suspected plant-parasitic nematodes of Rhode Island, II. Xiphinema americanum with notes on Tylencholaimus brevicaudatus n. comb. Proceedings of the Helminthological Society of Washington 23: 88-92.

Thorne G. 1939. A monograph of the nematodes of the superfamily Dorylaimoidea. Capita Zoologica 8: $1-261$.

Thorne G. 1974. Nematodes of the Northern Great Plains part II. Dorylaimoidea in part (Nematoda: Adenophorea). Agricultural Experiment Station, South Dakota State University, Technical Bulletin 41: 1-120. Available from https://openprairie.sdstate.edu/agexperimentsta_tb/5/ [accessed 11 Aug. 2021].

Vinciguerra M.T. 1986. New and known species of Tylencholaimus de Man, 1876 (Dorylaimida: Nematoda) from Italian beech forests with a key to the species. Nematologia Mediterranea 14: 107-116.

Wu W.J., Yu L., Xie H., Xu C.L., Yu J. \& Wang D.W. 2018. Description and molecular analysis of Tylencholaimus helanensis sp. n. from China (Dorylaimida, Tylencholaimidea). ZooKeys 792: 1-14. https://doi.org/10.3897/zookeys.792.27255

Wu W.J., Xu C.L., Xie H. \& Wang D.W. 2019. Three new species, one new genus and subfamily of Dorylaimida (de Man, 1876) Pearse, 1942, and revisions on the families Tylecholaimellidae Jairajpuri, 1964 and Mydonomidae Thorne, 1964 (Nematoda: Dorylaimida). PeerJ 7541: 1-30. https://doi.org/10.7717/peerj.7541

Yeates G.W. 1979. Nine new Dorylaimida (Nematoda) from the New Zealand region. Nematologica 25: 419-438. https://doi.org/10.1163/187529279X00587

Manuscript received: 31 March 2021

Manuscript accepted: 19 July 2021

Published on: 7 October 2021

Topic editor: Tony Robillard

Section editor: Martin Vinther Sørensen

Desk editor: Pepe Fernández

Printed versions of all papers are also deposited in the libraries of the institutes that are members of the EJT consortium: Muséum national d'histoire naturelle, Paris, France; Meise Botanic Garden, Belgium; Royal Museum for Central Africa, Tervuren, Belgium; Royal Belgian Institute of Natural Sciences, Brussels, Belgium; Natural History Museum of Denmark, Copenhagen, Denmark; Naturalis Biodiversity Center, Leiden, the Netherlands; Museo Nacional de Ciencias Naturales-CSIC, Madrid, Spain; Real Jardín Botánico de Madrid CSIC, Spain; Zoological Research Museum Alexander Koenig, Bonn, Germany; National Museum, Prague, Czech Republic. 